Supporting information

\title{
Mechanism of the Glutathione Persulfide Oxidation Process Catalyzed by Ethylmalonic Encephalopathy Protein 1
}

Beibei Lin, Guangcai Ma, Yongjun Liu*

School of Chemistry and Chemical Engineering, Shandong University, Jinan, Shandong 250100, China

Corresponding Author: Tel.: +86 53188365576; fax: +86 53188564464. Email address: yongjunliu_1@sdu.edu.cn (Y. Liu).

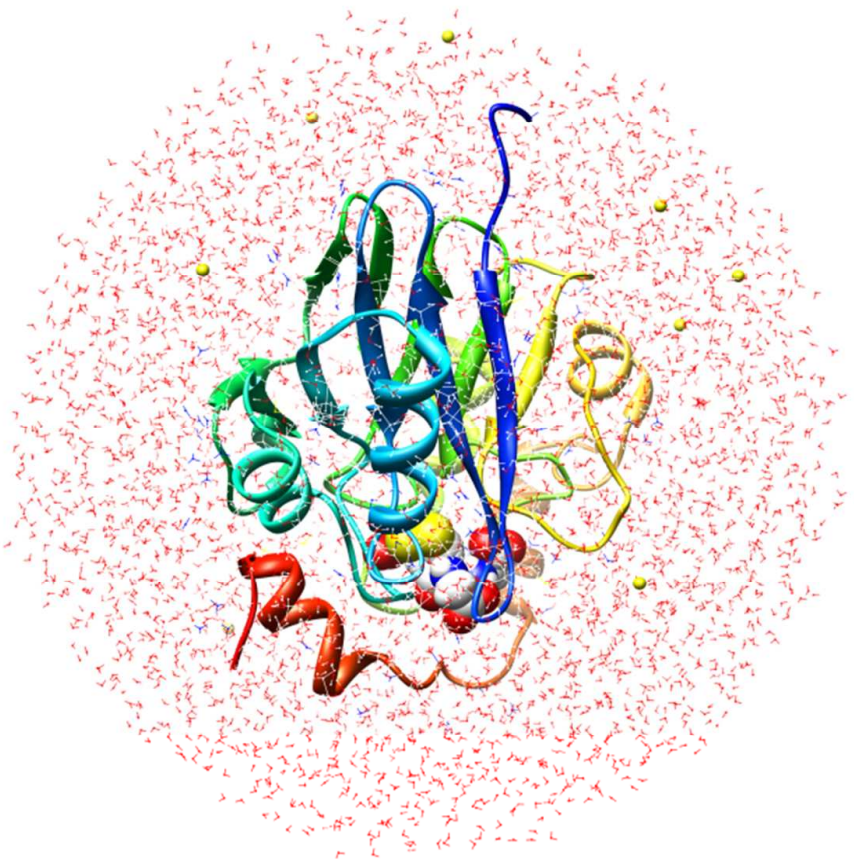

Figure S1 The constructed solvation model for the MD simulation. The small yellow balls represent the $\mathrm{Na}^{+}$ions and the big ball model shows the position of active site. 


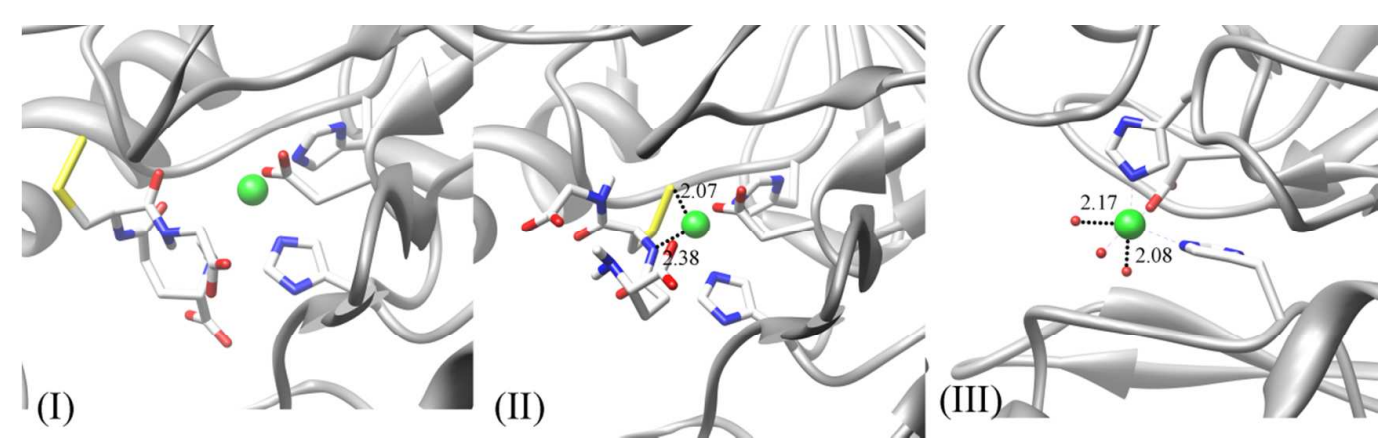

Figure S2 (I) and (II): two representative conformations after docking the substrate GSSH into hETHE1 active site. (III) : active site of crystal structure.

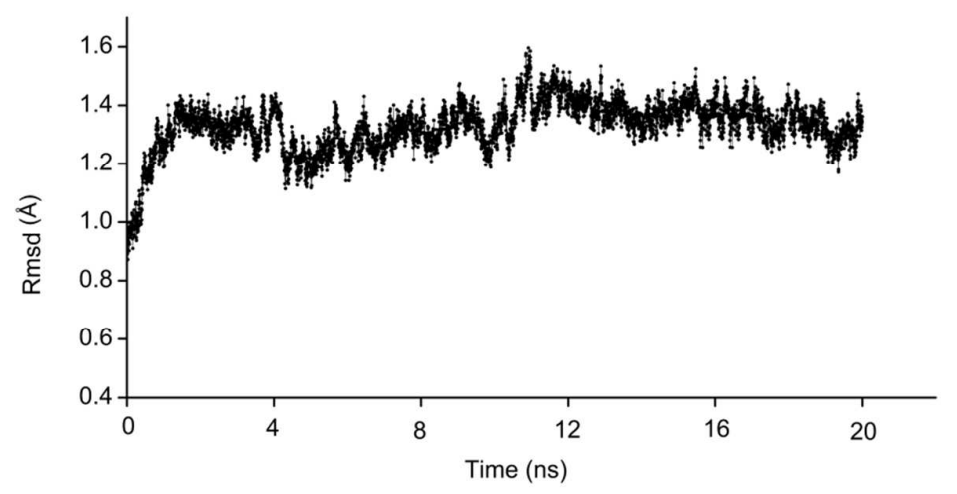

Figure S3 RMSD for the backbone atoms of the enzyme-substrate complex in 20-ns MD simulation.

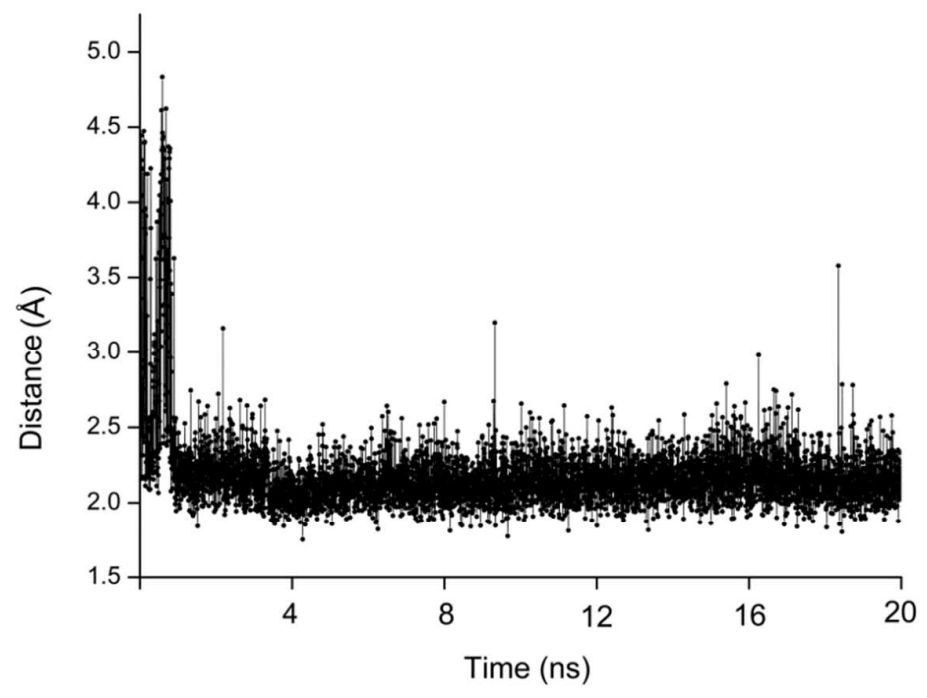

Figure S4 Distances between the H atom of the His81 and the S atom of substrate GSSH in 20-ns MD simulation. 


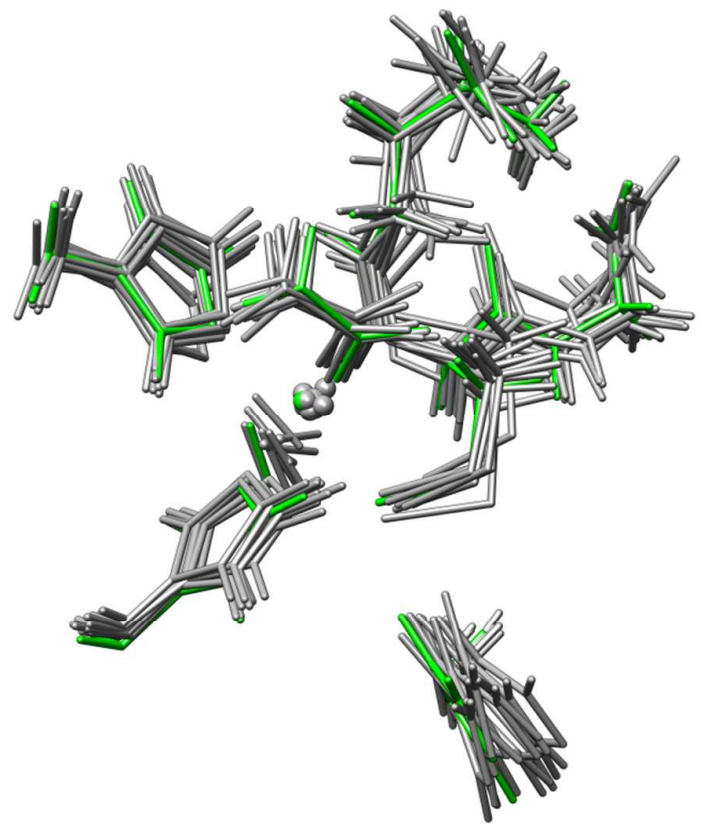

Figure S5 Overlay of QM/MM optimized geometries of ten snapshots from the 20-ns MD simulation at B1 level. An average structure was firstly derived and then the RMSDs of these ten structures relative to the average one were obtained, which are $0.49,0.62,0.36,0.41,0.38,0.36$, $0.43,0.53,0.58,0.35 \AA$ for $11,12,13,14,15,16,17,18,19,20 \mathrm{~ns}$, respectively. In general, the structure (shown in green) at $20 \mathrm{~ns}$ corresponds to the smallest RMSD value, and is considered to be representative, which was chosen for the QM/MM calculation.

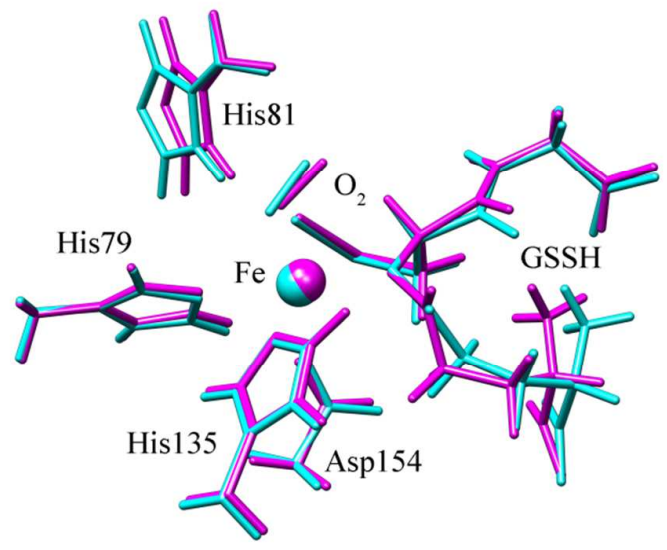

Figure S6 Overlay of the optimized geometries of model I (in cyan) and model II (in purple). 


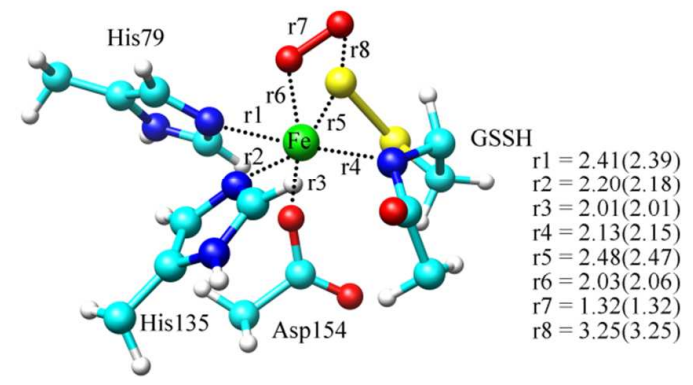

Figure S7 Optimized geometries of the reactant (enzyme-substrate complex) with basis sets of 6-31G(d,p)/Wachters $+\mathrm{f}(\mathrm{B} 1)$ and 6-31++G(d,p) /Wachters $+\mathrm{f}\left(\mathrm{B} 1^{\prime}\right)$. Key distances of reactant are given at the right.

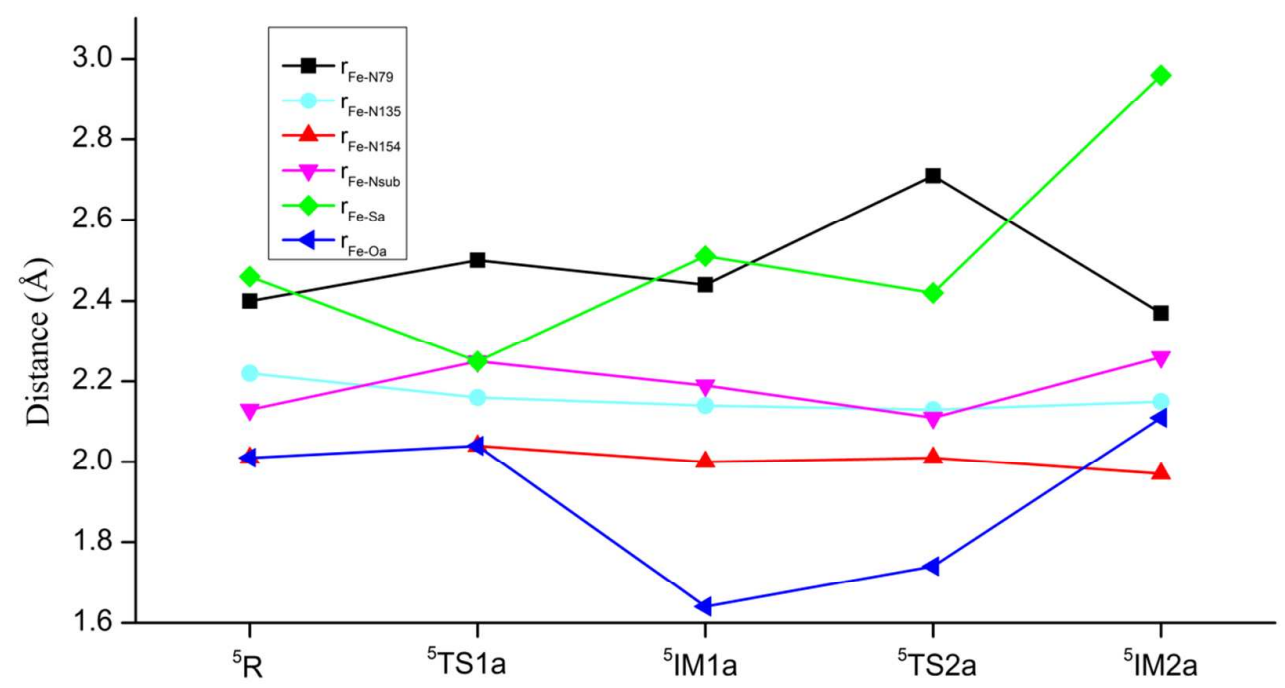

Figure S8 Distances between Fe ion and its ligating atoms for various species in Path_a. 


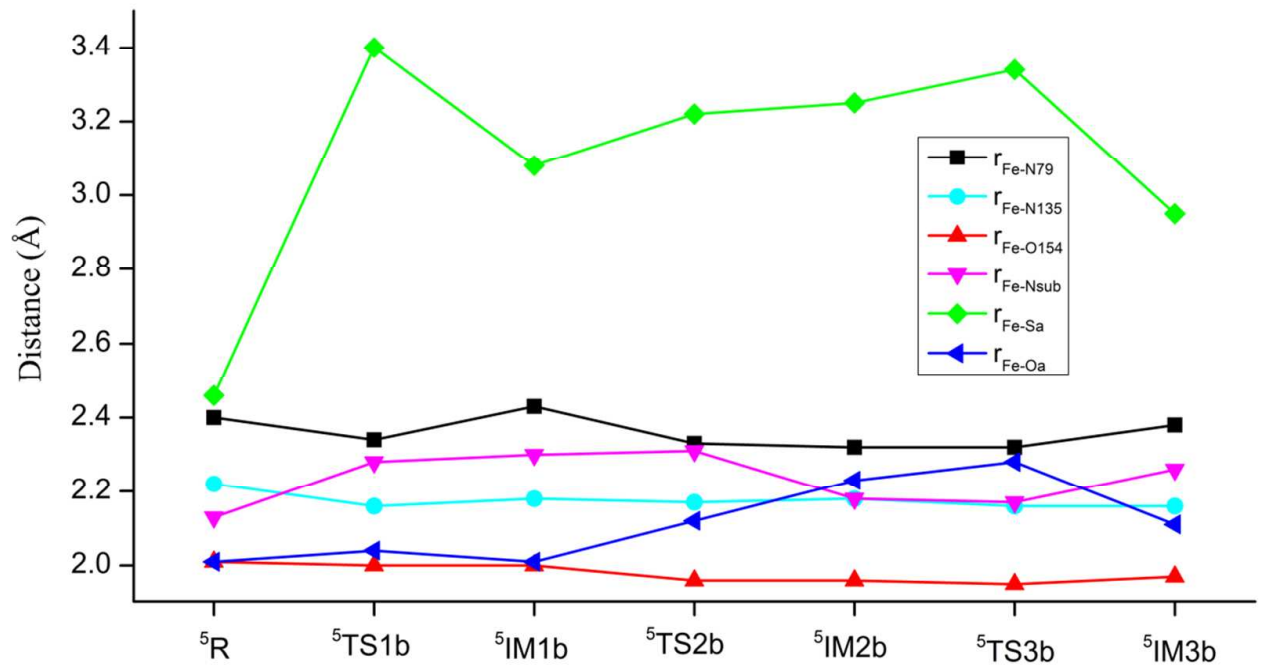

Figure S9 Distances between Fe ion and its ligating atoms for various species in Path_b.

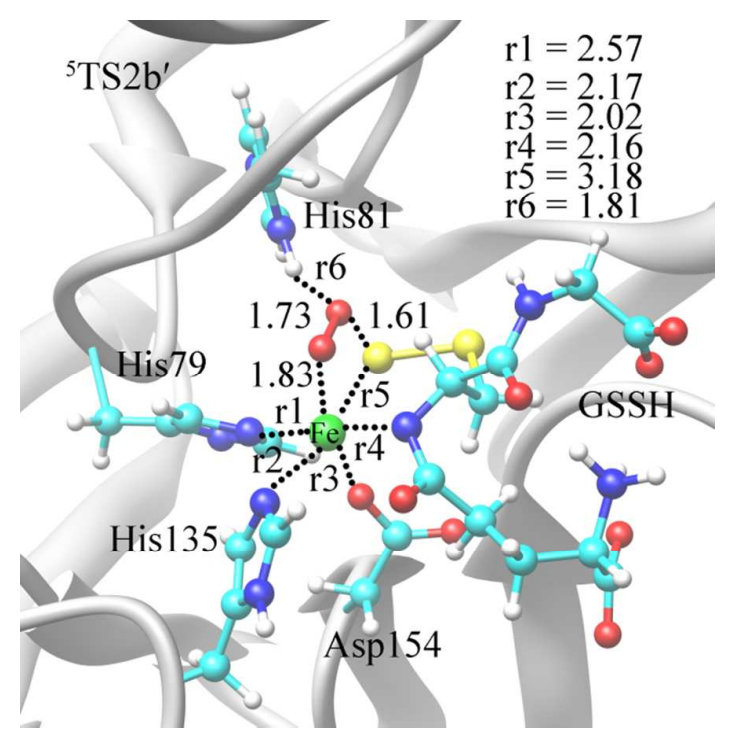

Figure S10 Optimized geometry for ${ }^{5} \mathrm{TS} 2 \mathrm{~b}^{\prime}$. All the distances are in angstroms. Dot lines represent coordination or hydrogen bonding. Some key distances are either listed in the figure directly, or shown at the upper right corner. 


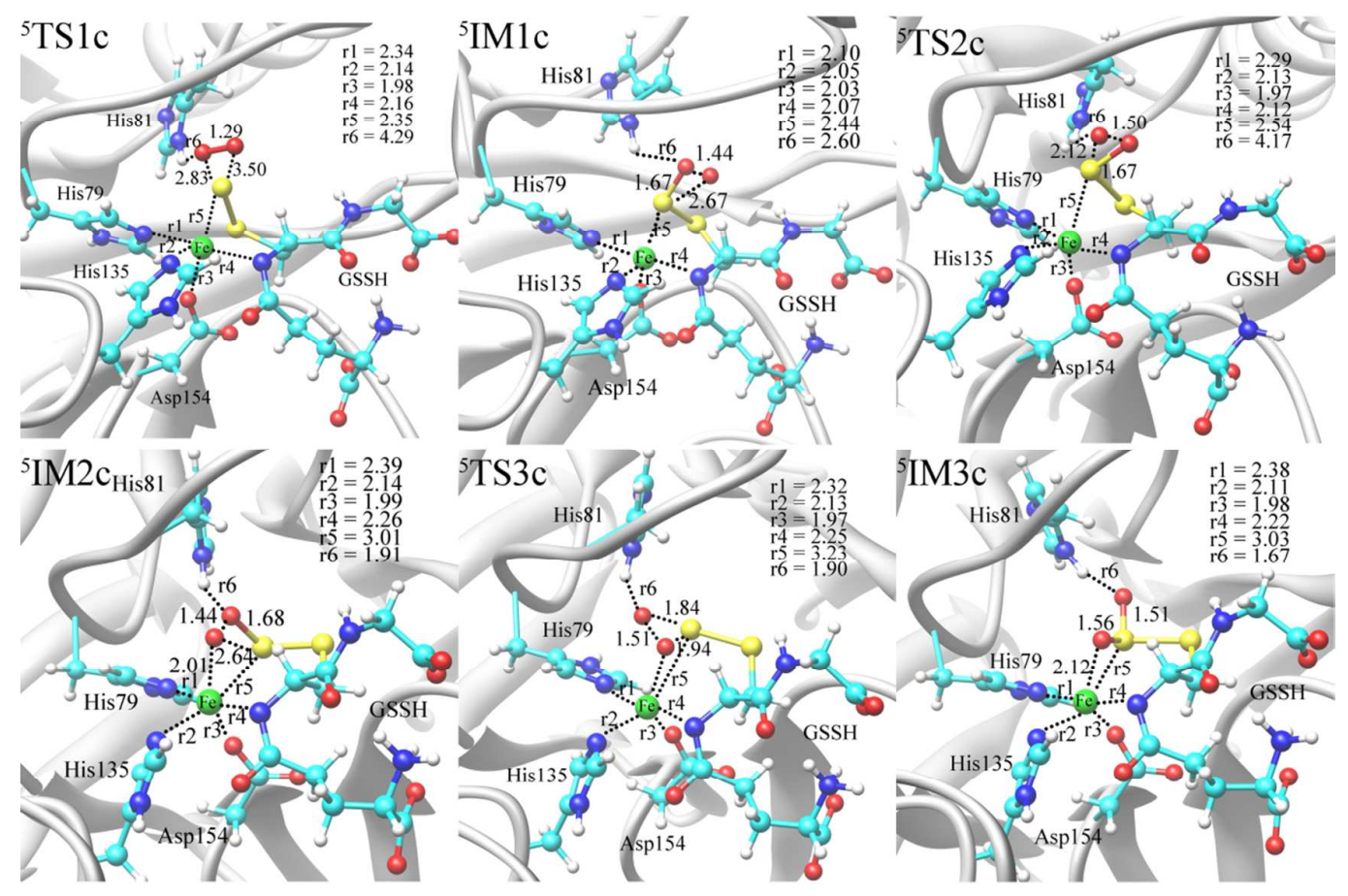

Figure S11 Optimized geometries for various species in Path_c with model II. All the distances are in angstroms. Dot lines represent coordination or hydrogen bonding. Some key distances are either listed in the figure directly, or shown at the upper right corner. 


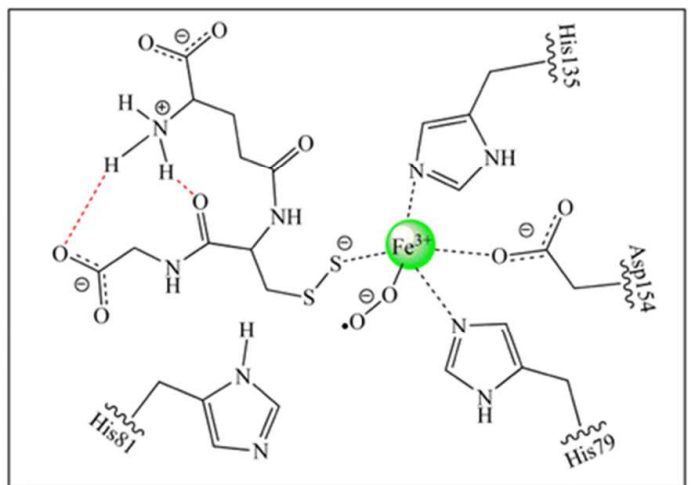

Figure S12 The QM-region model in the QM/MM calculations (model SI). Iron coordination bonds are shown in black dash lines and possible hydrogen bonds in red dash lines.

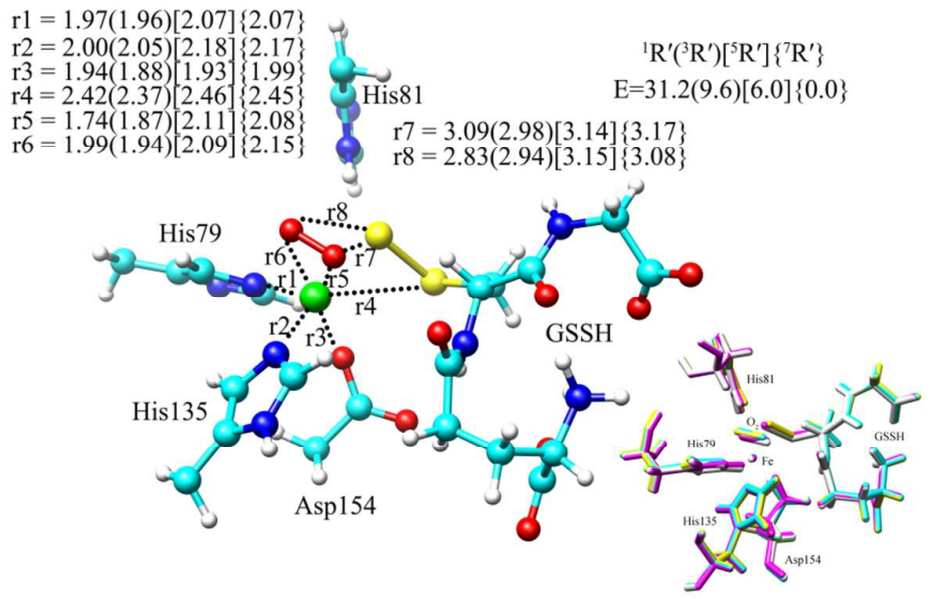

Figure S13 Optimized geometries of the reactant (enzyme-substrate complex) with four possible spin states. The relative energies $(\mathrm{kcal} / \mathrm{mol})$ are given for the singlet (triplet) [quintet] \{septet\} spin states. Structures depicted here were optimized using model SI at B1 level and energies were calculated at B2 level. All the distances are shown in angstroms. The lower-right corner is the overlay of the optimized geometries at different spin states. 


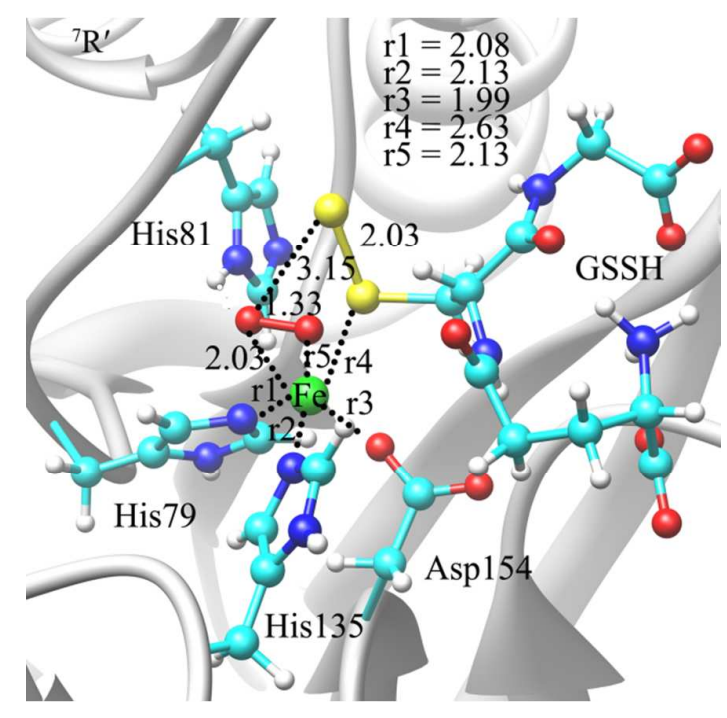

Figure S14 Optimized structure of reactant with Model SI. All the distances are in angstroms.

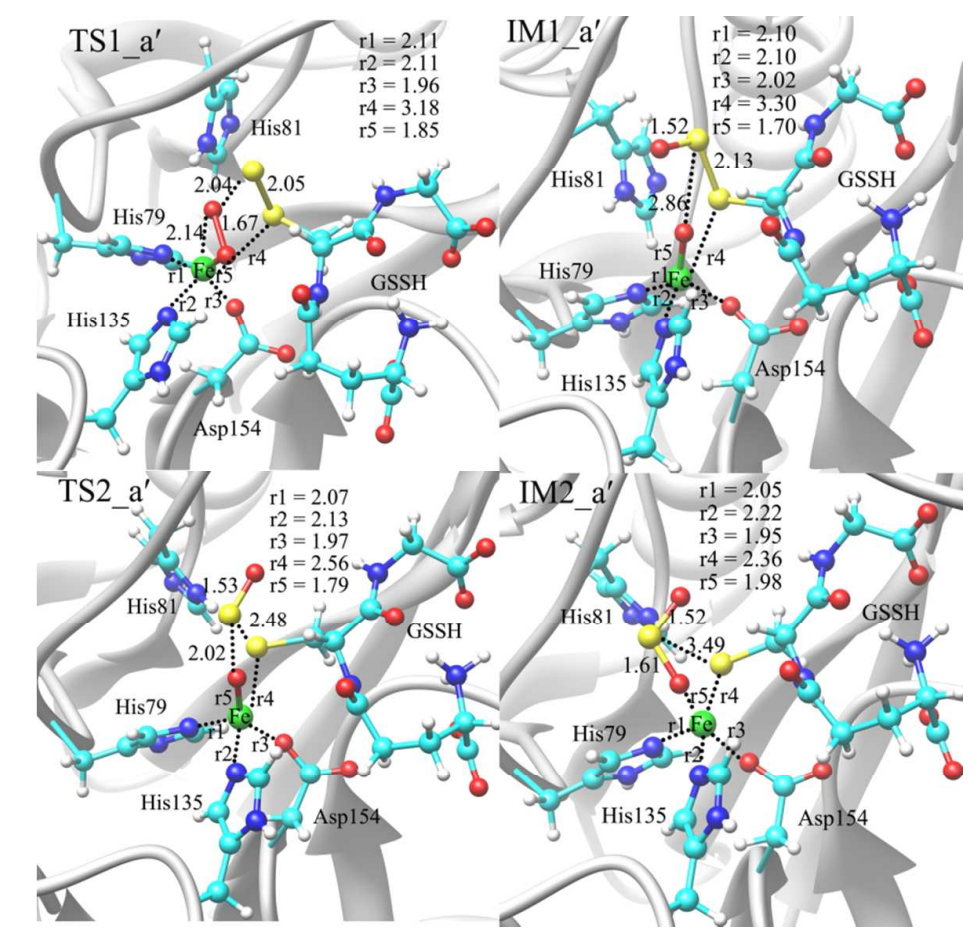

Figure S15 Optimized geometries for various species in Path_a' with model SI. All the distances are in angstroms. 


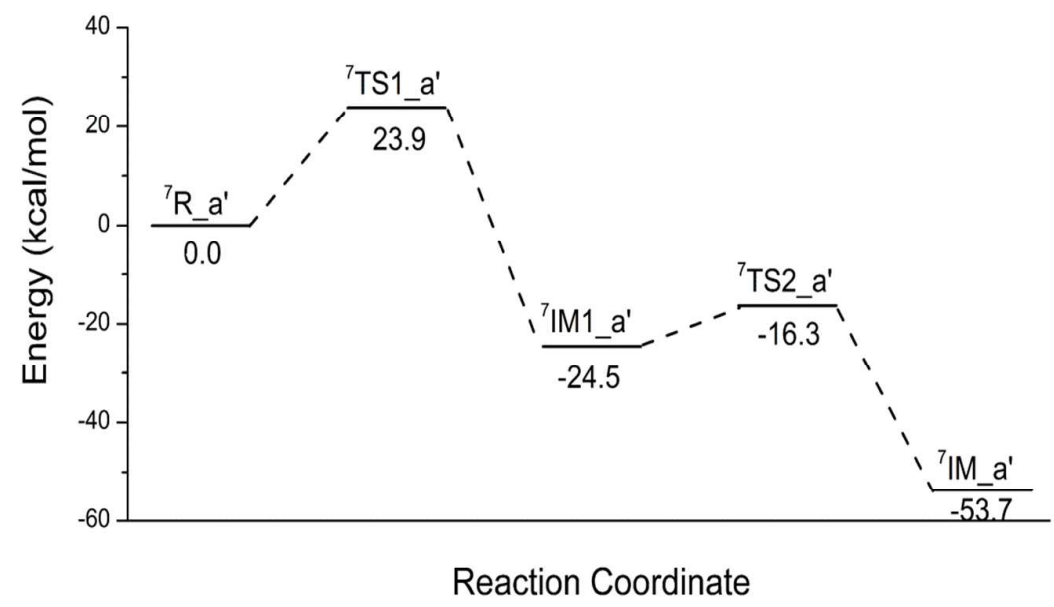

Figure S16 Energy profile of the GSSH oxidation catalyzed by ETHE1 at B2 level in Path_a'. The energy of the reactant at quintet state was set to zero.

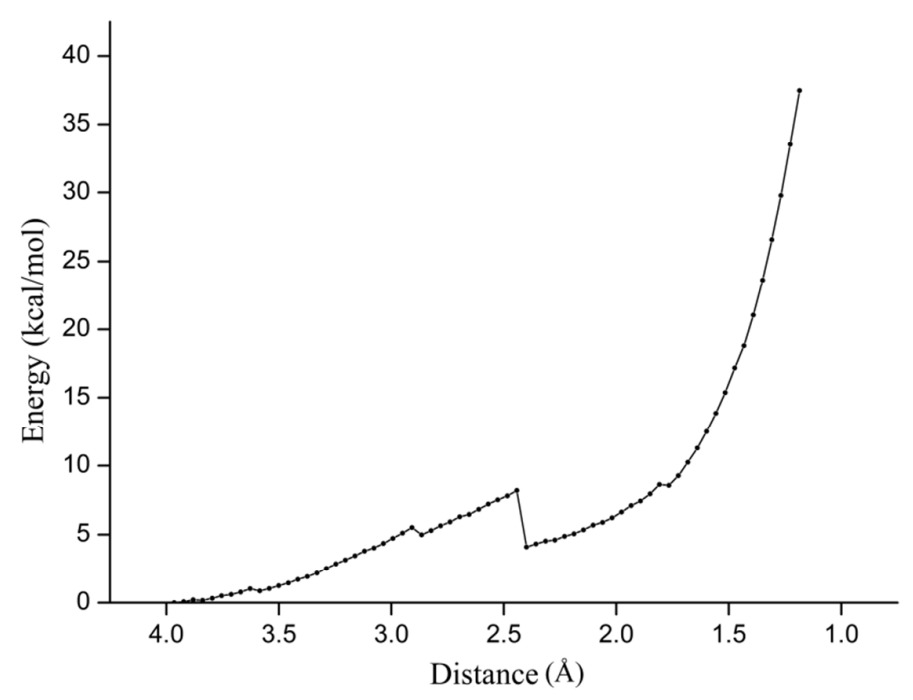

Figure S17 Scanned energy profile for proton transfer from His81 to the proximal O atom of $\mathrm{Fe}-\mathrm{O}_{2}$. The abscissa refers to the distance between the proton of His 81 and the proximal $\mathrm{O}$ atom of $\mathrm{Fe}-\mathrm{O}_{2}$. 


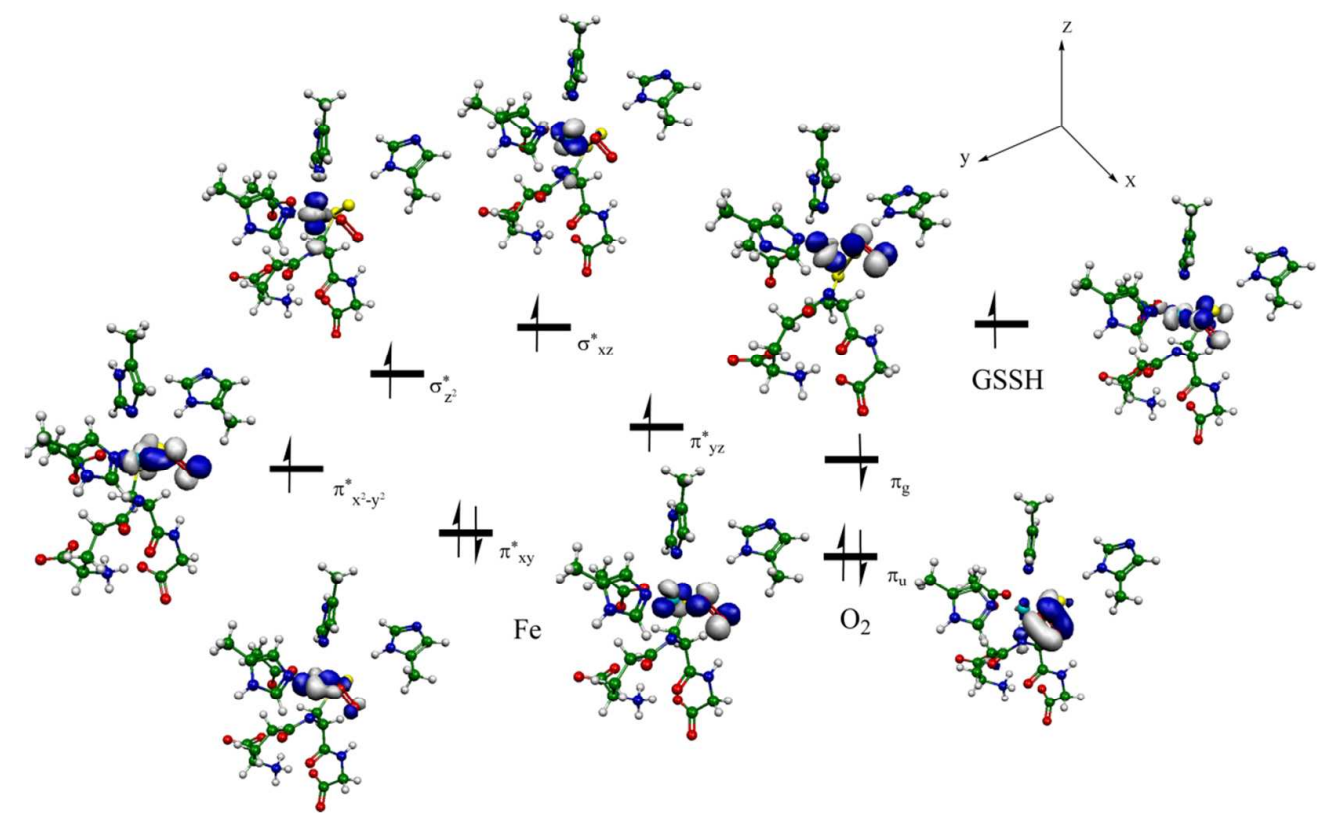

Figure S18 The valence electron orbital diagrams of ${ }^{5} \mathrm{R}$.

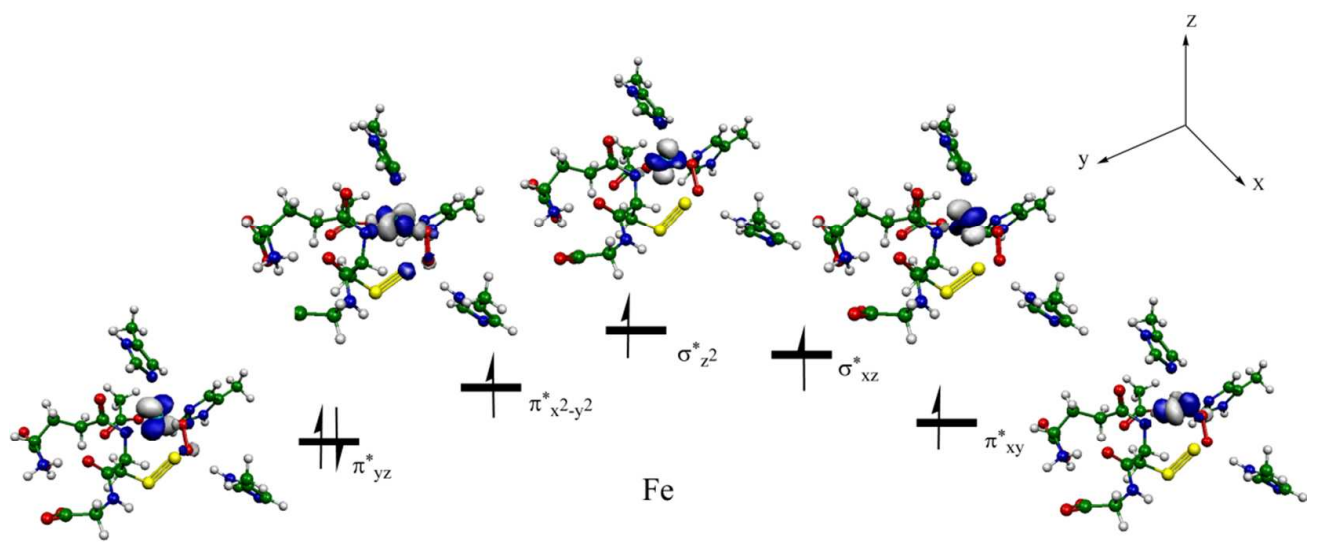

Figure S19The valence electron orbital diagrams of ${ }^{5} \mathrm{TS} 1 \mathrm{a}$. 


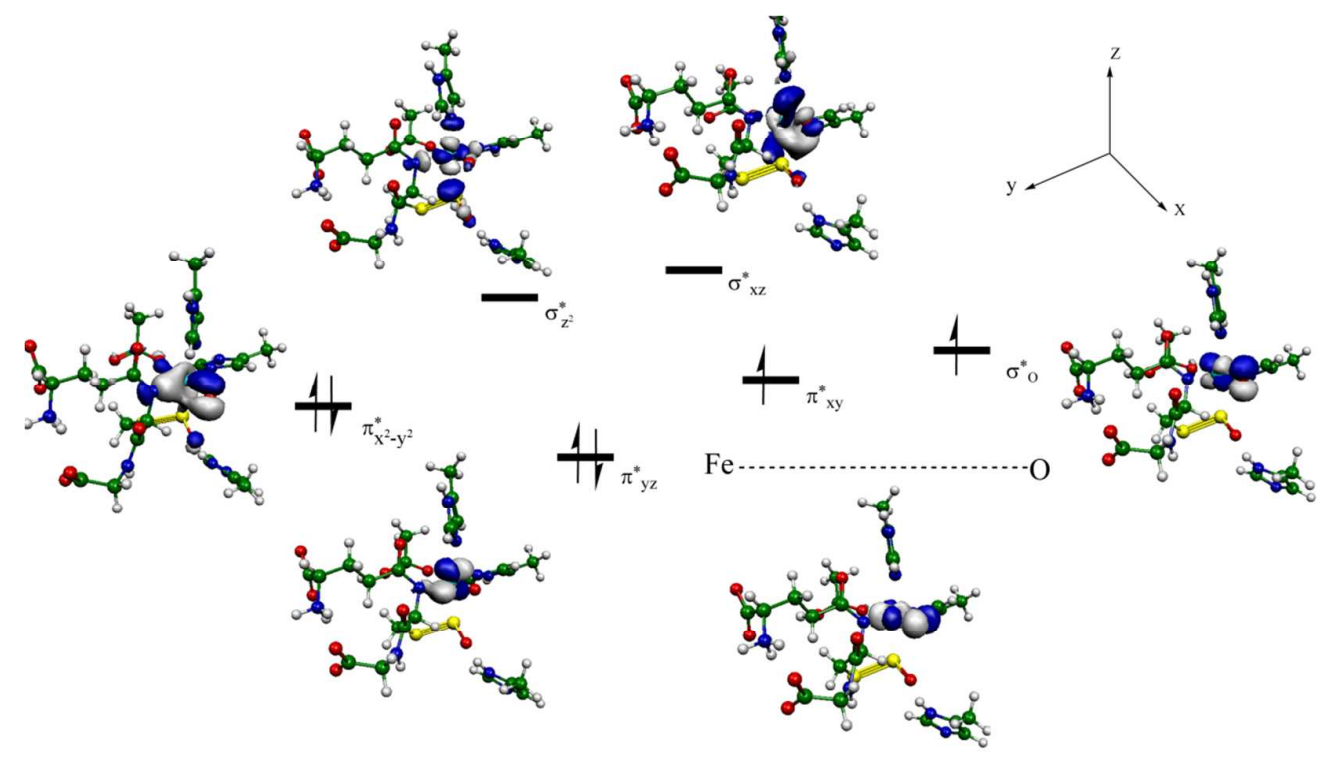

Figure S20 The valence electron orbital diagrams of ${ }^{3} \mathrm{IM} 1 \mathrm{a}$.

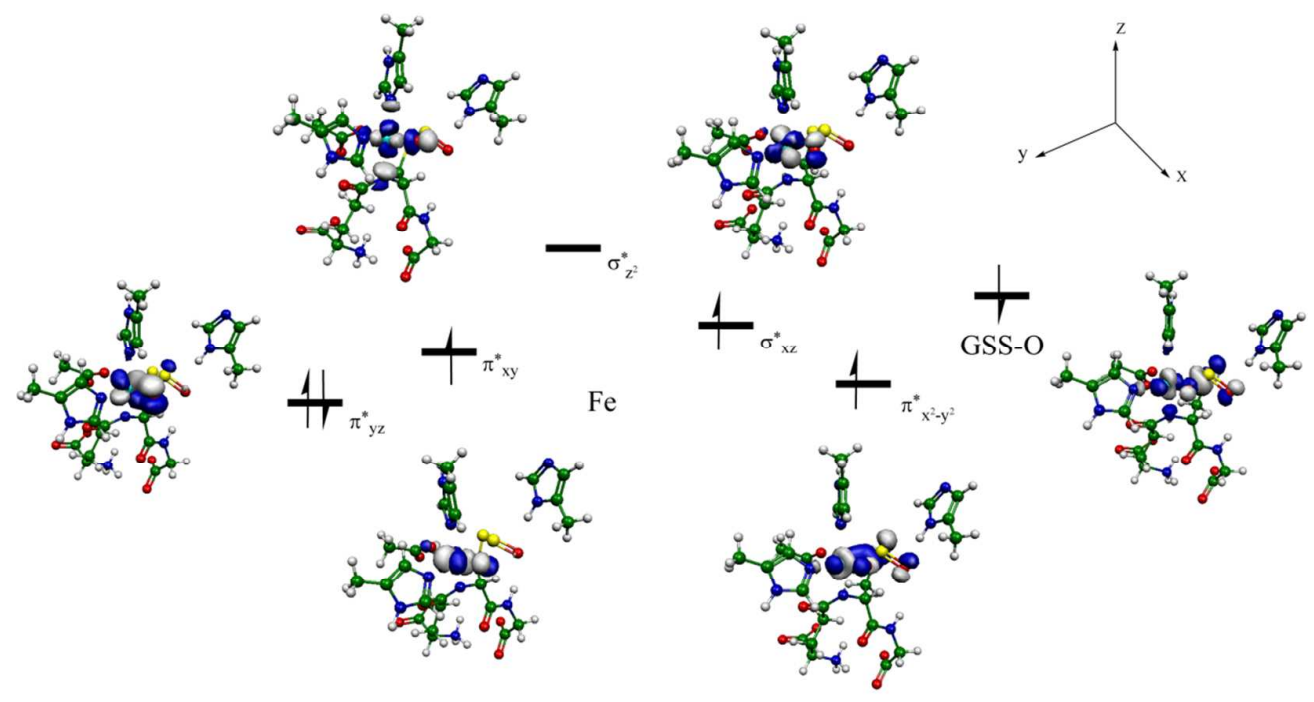

Figure S21 The valence electron orbital diagrams of ${ }^{3} \mathrm{TS} 2 \mathrm{a}$. 


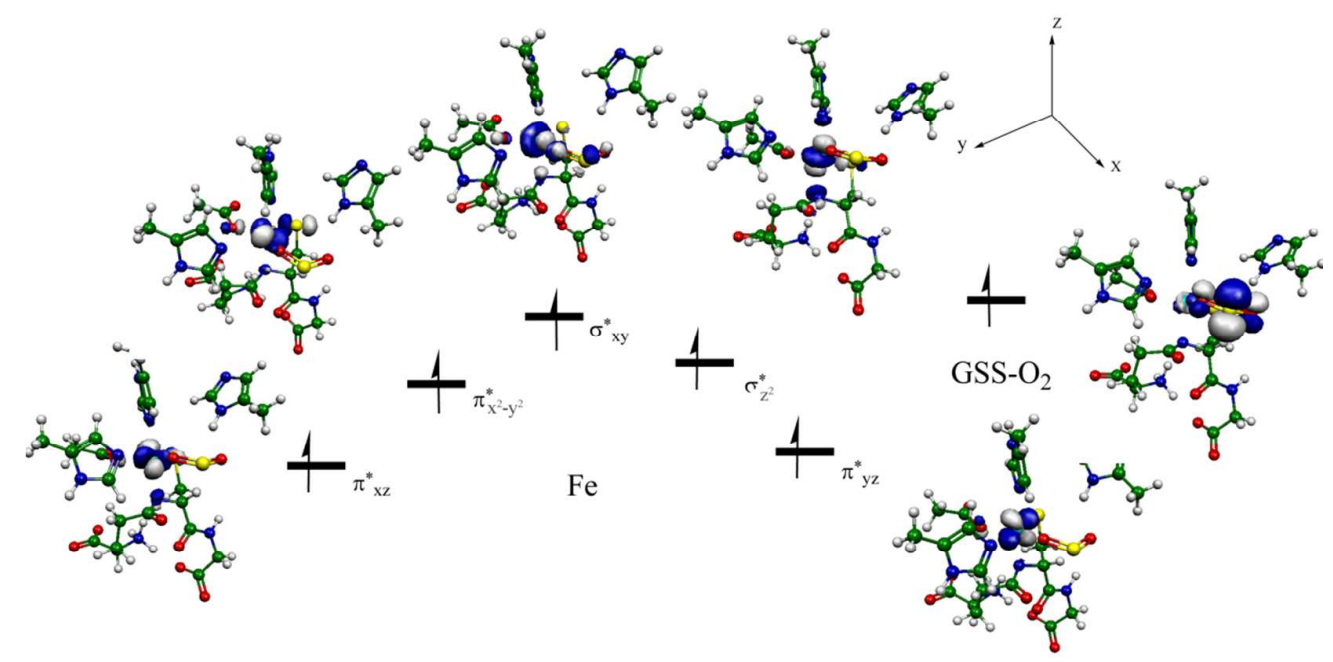

Figure S22 The valence electron orbital diagrams of ${ }^{7}$ IM2a. $^{2}$

Table S1 Key distances in the optimized geometries of ten snapshots from the 20-ns MD simulation calculated at B3LYP/B1 level.

\begin{tabular}{ccccccc}
\hline Species & $\mathrm{r}(\mathrm{Fe}-\mathrm{His} 79)$ & $\mathrm{r}(\mathrm{Fe}-\mathrm{His} 135)$ & $\mathrm{r}(\mathrm{Fe}-\mathrm{Asp} 154)$ & $\mathrm{r}(\mathrm{Fe}-\mathrm{N})$ & $\mathrm{r}(\mathrm{Fe}-\mathrm{S})$ & $\mathrm{r}(\mathrm{Fe}-\mathrm{O})$ \\
\hline $\mathrm{R} 11 \mathrm{~ns}$ & 2.26 & 2.20 & 2.05 & 2.11 & 2.47 & 2.02 \\
$\mathrm{R} 12 \mathrm{~ns}$ & 2.27 & 2.19 & 2.07 & 2.07 & 2.45 & 2.03 \\
$\mathrm{R} 13 \mathrm{~ns}$ & 2.28 & 2.25 & 2.02 & 2.06 & 2.48 & 2.02 \\
$\mathrm{R} 14 \mathrm{~ns}$ & 2.19 & 2.34 & 2.09 & 2.05 & 2.53 & 2.00 \\
R15ns & 2.20 & 2.18 & 2.03 & 2.15 & 2.58 & 1.96 \\
R16ns & 2.25 & 2.33 & 2.13 & 2.07 & 2.67 & 2.09 \\
R17ns & 2.19 & 2.21 & 2.02 & 2.12 & 2.55 & 1.95 \\
R18ns & 2.21 & 2.23 & 2.08 & 2.07 & 2.49 & 2.20 \\
R19ns & 2.29 & 2.27 & 2.05 & 2.11 & 2.41 & 1.95 \\
R20ns & 2.40 & 2.22 & 2.01 & 2.13 & 2.46 & 2.01 \\
$\overline{\mathrm{R}}$ & 2.25 & 2.24 & 2.05 & 2.10 & 2.50 & 2.02 \\
\hline
\end{tabular}


Table S2 Natural Population Atomic Charges of selected atoms in species for the ETHE1-substrate complex in Path_a with model II calculated at B3LYP/B2 level.

\begin{tabular}{cccccc}
\hline Atoms/Species & ${ }^{5} \mathrm{R}$ & ${ }^{5}$ TS1a & ${ }^{5}$ IM1a & ${ }^{5}$ TS2a & ${ }^{5}$ IM2a \\
\hline $\mathrm{Fe}$ & 1.49 & 1.34 & 1.37 & 1.45 & 1.38 \\
$\mathrm{~S}_{\mathrm{a}}$ & -0.47 & -0.06 & 0.59 & 0.86 & 1.40 \\
$\mathrm{O}_{\mathrm{a}}$ & -0.42 & -0.50 & -0.60 & -0.84 & -1.00 \\
$\mathrm{O}_{154}$ & -0.86 & -0.88 & -0.86 & -0.86 & -0.88 \\
$\mathrm{~N}_{79}$ & -0.56 & -0.54 & -0.54 & -0.53 & -0.56 \\
$\mathrm{~N}_{135}$ & -0.60 & -0.61 & -0.57 & -0.60 & -0.61 \\
$\mathrm{~N}_{\text {sub }}$ & -0.78 & -0.81 & -0.77 & -0.79 & -0.84 \\
\hline
\end{tabular}

Table S3 Natural Population Atomic Charges of selected atoms in species for the ETHE1-substrate complex in Path_b with model II calculated at B3LYP/B2 level.

\begin{tabular}{cccccccc}
\hline Atoms/Species & ${ }^{5} \mathrm{R}$ & ${ }^{5} \mathrm{TS} 1 \mathrm{~b}$ & ${ }^{5} \mathrm{IM} 1 \mathrm{~b}$ & ${ }^{5} \mathrm{TS} 2 \mathrm{~b}$ & ${ }^{5} \mathrm{IM} 2 \mathrm{~b}$ & ${ }^{5} \mathrm{TS} 3 \mathrm{~b}$ & ${ }^{5} \mathrm{IM} 3 \mathrm{~b}$ \\
\hline $\mathrm{Fe}$ & 1.49 & 1.43 & 1.41 & 1.45 & 1.45 & 1.44 & 1.38 \\
$\mathrm{~S}_{\mathrm{a}}$ & -0.47 & -0.21 & 0.33 & 0.37 & 0.28 & 0.38 & 1.40 \\
$\mathrm{O}_{\mathrm{a}}$ & -0.42 & -0.46 & -0.64 & -0.58 & -0.54 & -0.55 & -1.00 \\
$\mathrm{O}_{154}$ & -0.86 & -0.89 & -0.89 & -0.90 & -0.90 & -0.90 & -0.88 \\
$\mathrm{~N}_{79}$ & -0.56 & -0.61 & -0.61 & -0.62 & -0.62 & -0.62 & -0.61 \\
$\mathrm{~N}_{135}$ & -0.60 & -0.56 & -0.55 & -0.57 & -0.57 & -0.58 & -0.56 \\
$\mathrm{~N}_{\text {sub }}$ & -0.78 & -0.83 & -0.83 & -0.85 & -0.87 & -0.88 & -0.85 \\
\hline
\end{tabular}

Table S4 Natural Population Atomic Charges of selected atoms in species for the ETHE1-substrate complex in Path_c with model II calculated at B3LYP/B2 level.

\begin{tabular}{cccccccc}
\hline Atoms/Species & ${ }^{5} \mathrm{R}$ & ${ }^{5} \mathrm{TS} 1 \mathrm{~b}$ & ${ }^{5} \mathrm{IM} 1 \mathrm{~b}$ & ${ }^{5} \mathrm{TS} 2 \mathrm{~b}$ & ${ }^{5} \mathrm{IM} 2 \mathrm{~b}$ & ${ }^{5} \mathrm{TS} 3 \mathrm{~b}$ & ${ }^{5} \mathrm{IM} 3 \mathrm{c}$ \\
\hline $\mathrm{Fe}$ & 1.49 & 1.30 & 1.26 & 1.31 & 1.35 & 1.39 & 1.42 \\
$\mathrm{~S}_{\mathrm{a}}$ & -0.47 & -0.17 & 0.28 & 0.39 & 0.32 & 0.34 & 1.45 \\
$\mathrm{O}_{\mathrm{a}}$ & -0.42 & -0.46 & -0.48 & -0.50 & -0.49 & -0.55 & -0.98 \\
$\mathrm{O}_{154}$ & -0.86 & -0.86 & -0.87 & -0.88 & -0.87 & -0.88 & -0.87 \\
$\mathrm{~N}_{79}$ & -0.56 & -0.54 & -0.59 & -0.57 & -0.54 & -0.56 & -0.60 \\
$\mathrm{~N}_{135}$ & -0.60 & -0.61 & -0.64 & -0.65 & -0.63 & -0.64 & -0.66 \\
$\mathrm{~N}_{\text {sub }}$ & -0.78 & -0.81 & -0.80 & -0.81 & -0.80 & -0.81 & -0.85 \\
\hline
\end{tabular}


Table S5 Natural Population Atomic Charges of selected atoms in species for the ETHE1-substrate complex in Path_a considering spin-state crossover calculated at B3LYP/B2 level.

\begin{tabular}{cccccc}
\hline Atoms/Species & ${ }^{5} \mathrm{R}$ & ${ }^{5} \mathrm{TS} 1 \mathrm{a}$ & ${ }^{3} \mathrm{IM} 1 \mathrm{a}$ & ${ }^{3} \mathrm{TS} 2 \mathrm{a}$ & ${ }^{7}$ IM2a \\
\hline $\mathrm{Fe}$ & 1.49 & 1.34 & 0.92 & 1.09 & 1.51 \\
$\mathrm{~S}_{\mathrm{a}}$ & -0.47 & -0.06 & 0.64 & 0.85 & 1.16 \\
$\mathrm{O}_{\mathrm{a}}$ & -0.42 & -0.50 & -0.54 & -0.76 & -0.99 \\
$\mathrm{O}_{154}$ & -0.86 & -0.88 & -0.82 & -0.81 & -0.82 \\
$\mathrm{~N}_{79}$ & -0.56 & -0.54 & -0.50 & -0.51 & -0.58 \\
$\mathrm{~N}_{135}$ & -0.60 & -0.61 & -0.50 & -0.56 & -0.50 \\
$\mathrm{~N}_{\text {sub }}$ & -0.78 & -0.81 & -0.60 & -0.81 & -0.83 \\
\hline
\end{tabular}

Table S6 Spin density distribution for the GSSH-Fe- $\mathrm{O}_{2}$ complex in Path_a considering spin-state crossover calculated at B3LYP/B2 level.

\begin{tabular}{ccccccc}
\hline species & $\mathrm{Fe}$ & $\mathrm{O}_{\mathrm{a}}$ & $\mathrm{O}_{\mathrm{b}}$ & $\mathrm{S}_{\mathrm{a}}$ & $\mathrm{S}_{\mathrm{b}}$ & $\mathrm{N}_{\text {sub }}$ \\
\hline${ }^{5} \mathrm{R}$ & 4.05 & -0.20 & -0.57 & 0.21 & 0.07 & 0.18 \\
${ }^{5} \mathrm{TS} 1 \mathrm{a}$ & 3.64 & 0.01 & -0.11 & 0.17 & 0.08 & 0.06 \\
${ }^{3} \mathrm{IM} 1 \mathrm{a}$ & 1.52 & 0.62 & -0.04 & -0.05 & -0.01 & -0.05 \\
${ }^{3} \mathrm{TS} 2 \mathrm{a}$ & 2.45 & 0.08 & -0.13 & -0.25 & -0.11 & -0.08 \\
${ }^{7} \mathrm{IM} 2 \mathrm{a}$ & 4.12 & 0.27 & 0.33 & 0.51 & 0.29 & 0.16 \\
\hline
\end{tabular}

Table S7 The scanning radius from reactant to products in different pathways.

\begin{tabular}{cc}
\hline Elementary step & Reaction Coordinate \\
\hline R to IM1a & $r\left(\mathrm{O}_{\mathrm{a}}-\mathrm{O}_{\mathrm{b}}\right)-\mathrm{r}\left(\mathrm{O}_{\mathrm{b}}-\mathrm{S}_{\mathrm{a}}\right)$ \\
IM1a to IM2a & $\mathrm{r}\left(\mathrm{Fe}-\mathrm{O}_{\mathrm{a}}\right)-\mathrm{r}\left(\mathrm{O}_{\mathrm{a}}-\mathrm{S}_{\mathrm{a}}\right)$ \\
R to IM1b & $-\mathrm{r}\left(\mathrm{O}_{\mathrm{b}}-\mathrm{S}_{\mathrm{a}}\right)$ \\
IM1b to IM2b & $\mathrm{r}\left(\mathrm{Fe}-\mathrm{O}_{\mathrm{a}}\right)-\mathrm{r}\left(\mathrm{Oa}-\mathrm{S}_{\mathrm{a}}\right)$ \\
IM2b to IM3b & $-\mathrm{r}\left(\mathrm{O}_{\mathrm{b}}-\mathrm{S}_{\mathrm{a}}\right)$ \\
R to IM1c & $r\left(\mathrm{Fe}-\mathrm{O}_{\mathrm{a}}\right)-\mathrm{r}\left(\mathrm{O}_{\mathrm{a}}-\mathrm{S}_{\mathrm{a}}\right)$ \\
IM1c to IM2c & $-\mathrm{r}\left(\mathrm{O}_{\mathrm{b}}-\mathrm{S}_{\mathrm{a}}\right)$ \\
IM2c to IM3c & $r\left(\mathrm{O}_{\mathrm{a}}-\mathrm{O}_{\mathrm{b}}\right)-\mathrm{r}\left(\mathrm{O}_{\mathrm{b}}-\mathrm{S}_{\mathrm{a}}\right)$ \\
IM1b to IM1a & $r\left(\mathrm{O}_{\mathrm{a}}-\mathrm{O}_{\mathrm{b}}\right)$ \\
\hline
\end{tabular}




\section{Absolute QM/MM single-point energies, as well as Cartesian coordinates of QM regions of all computed species:}

\begin{tabular}{|c|c|c|c|c|c|c|c|}
\hline & for Model I & & & $\mathrm{s}$ & -4.19200000 & -5.70800000 & -3.46200000 \\
\hline & 328.092536 (a.u. & & & $\mathrm{s}$ & -4.08400000 & -6.75600000 & -1.67900000 \\
\hline C & -3.21000000 & -4.96900000 & 4.19800000 & $\mathrm{Fe}$ & -6.11400000 & -6.50500000 & -0.57500000 \\
\hline $\mathrm{H}$ & -3.77100000 & -5.63800000 & 4.85100000 & $\mathrm{O}$ & -5.83500000 & -9.12800000 & -0.80600000 \\
\hline $\mathrm{H}$ & -3.42400000 & -3.94900000 & 4.54200000 & $\mathrm{O}$ & -6.02200000 & -8.13700000 & 0.02600000 \\
\hline $\mathrm{N}$ & -3.34700000 & -4.33100000 & 1.72900000 & & & & \\
\hline $\mathrm{H}$ & -2.65500000 & -3.58700000 & 1.71600000 & ${ }^{3} R f$ & for Model I & & \\
\hline C & -3.68300000 & -5.16400000 & 2.79000000 & -332 & 28.130779 (а.u.) & & \\
\hline C & -4.06300000 & -4.70800000 & 0.65200000 & $\mathrm{C}$ & -3.11100000 & -4.97100000 & 4.25300000 \\
\hline $\mathrm{H}$ & -4.00000000 & -4.22800000 & -0.30800000 & $\mathrm{H}$ & -3.69900000 & -5.61800000 & 4.90500000 \\
\hline $\mathrm{N}$ & -4.85100000 & -5.73700000 & 0.94400000 & $\mathrm{H}$ & -3.30800000 & -3.94200000 & 4.57900000 \\
\hline C & -4.61400000 & -6.03500000 & 2.27100000 & $\mathrm{~N}$ & -3.13500000 & -4.43800000 & 1.74600000 \\
\hline $\mathrm{H}$ & -5.10100000 & -6.86400000 & 2.75700000 & $\mathrm{H}$ & -2.40900000 & -3.72200000 & 1.71100000 \\
\hline C & -9.80900000 & -3.64900000 & 2.51800000 & $\mathrm{C}$ & -3.55700000 & -5.18500000 & 2.84000000 \\
\hline $\mathrm{H}$ & -10.35300000 & -2.97500000 & 1.84200000 & C & -3.84000000 & -4.83400000 & 0.67000000 \\
\hline $\mathrm{H}$ & -8.99700000 & -3.06100000 & 2.95700000 & $\mathrm{H}$ & -3.71900000 & -4.41000000 & -0.31200000 \\
\hline $\mathrm{N}$ & -9.85500000 & -5.91400000 & 1.29100000 & $\mathrm{~N}$ & -4.70400000 & -5.78900000 & 0.99300000 \\
\hline $\mathrm{H}$ & -10.87000000 & -6.04200000 & 1.31200000 & $\mathrm{C}$ & -4.52500000 & -6.02500000 & 2.33900000 \\
\hline C & -9.18800000 & -4.79900000 & 1.77800000 & $\mathrm{H}$ & -5.07800000 & -6.79200000 & 2.85600000 \\
\hline C & -8.95700000 & -6.69800000 & 0.65100000 & C & -9.77300000 & -3.61500000 & 2.61600000 \\
\hline $\mathrm{H}$ & -9.20600000 & -7.63100000 & 0.17200000 & $\mathrm{H}$ & -10.30800000 & -2.94500000 & 1.92800000 \\
\hline $\mathrm{N}$ & -7.75500000 & -6.13700000 & 0.66500000 & $\mathrm{H}$ & -8.98100000 & -3.01600000 & 3.07800000 \\
\hline C & -7.88500000 & -4.97300000 & 1.39400000 & $N$ & -9.77900000 & -5.83700000 & 1.32100000 \\
\hline $\mathrm{H}$ & -7.03600000 & -4.33500000 & 1.56900000 & $\mathrm{H}$ & -10.79600000 & -5.94200000 & 1.27300000 \\
\hline C & -7.17600000 & -2.32600000 & -0.70000000 & C & -9.12100000 & -4.75200000 & 1.88700000 \\
\hline $\mathrm{H}$ & -6.98200000 & -2.52800000 & 0.35400000 & $\mathrm{C}$ & -8.86300000 & -6.59600000 & 0.68900000 \\
\hline $\mathrm{H}$ & -8.26000000 & -2.35100000 & -0.85600000 & $\mathrm{H}$ & -9.10800000 & -7.47800000 & 0.11900000 \\
\hline C & -6.60700000 & -3.45500000 & -1.59700000 & $\mathrm{~N}$ & -7.64800000 & -6.09000000 & 0.83600000 \\
\hline $\mathrm{O}$ & -6.61300000 & -3.29800000 & -2.82800000 & $\mathrm{C}$ & -7.80000000 & -4.94500000 & 1.58800000 \\
\hline $\mathrm{O}$ & -6.27500000 & -4.49500000 & -0.93600000 & $\mathrm{H}$ & -6.95000000 & -4.33500000 & 1.84500000 \\
\hline C & -9.06200000 & -5.41900000 & -2.87400000 & C & -7.06400000 & -2.33100000 & -0.61000000 \\
\hline $\mathrm{H}$ & -9.48700000 & -4.97900000 & -1.95600000 & $\mathrm{H}$ & -6.85800000 & -2.50700000 & 0.44700000 \\
\hline $\mathrm{H}$ & -8.25400000 & -4.75200000 & -3.17200000 & $\mathrm{H}$ & -8.14000000 & -2.43000000 & -0.76700000 \\
\hline C & -8.57600000 & -6.78800000 & -2.37100000 & C & -6.41200000 & -3.42700000 & -1.47800000 \\
\hline $\mathrm{O}$ & -9.45900000 & -7.60700000 & -2.05700000 & $\mathrm{O}$ & -6.34400000 & -3.28100000 & -2.70600000 \\
\hline $\mathrm{N}$ & -7.21800000 & -7.11700000 & -2.19900000 & $\mathrm{O}$ & -6.02400000 & -4.44000000 & -0.79400000 \\
\hline C & -6.40600000 & -7.50400000 & -3.36800000 & C & -8.57200000 & -5.10200000 & -3.81800000 \\
\hline $\mathrm{H}$ & -5.55700000 & -8.03000000 & -2.92300000 & $\mathrm{H}$ & -8.49500000 & -4.01700000 & -3.70900000 \\
\hline C & -5.82200000 & -6.30300000 & -4.16300000 & $\mathrm{H}$ & -7.81100000 & -5.37600000 & -4.54900000 \\
\hline $\mathrm{H}$ & -6.46400000 & -5.42400000 & -4.07700000 & C & -8.27000000 & -5.67300000 & -2.43900000 \\
\hline $\mathrm{H}$ & -5.60100000 & -6.49200000 & -5.22200000 & 0 & -8.99200000 & -5.25900000 & -1.51400000 \\
\hline
\end{tabular}




\begin{tabular}{|c|c|c|c|c|c|c|c|}
\hline $\mathrm{N}$ & -7.25300000 & -6.59400000 & -2.23300000 & $\mathrm{H}$ & -7.91500000 & -5.33500000 & -4.49300000 \\
\hline C & -6.44000000 & -7.12600000 & -3.35300000 & $\mathrm{C}$ & -8.50500000 & -6.02300000 & -2.53600000 \\
\hline $\mathrm{H}$ & -5.80700000 & -7.87300000 & -2.86800000 & 0 & -9.37600000 & -5.96400000 & -1.63300000 \\
\hline C & -5.50900000 & -6.09200000 & -4.02500000 & $\mathrm{~N}$ & -7.37900000 & -6.78900000 & -2.36700000 \\
\hline $\mathrm{H}$ & -5.95200000 & -5.10300000 & -3.97800000 & $\mathrm{C}$ & -6.49200000 & -7.21100000 & -3.46800000 \\
\hline $\mathrm{H}$ & -5.25000000 & -6.32900000 & -5.06300000 & $\mathrm{H}$ & -5.83800000 & -7.94800000 & -2.99300000 \\
\hline S & -3.87800000 & -5.84700000 & -3.14300000 & $\mathrm{C}$ & -5.59100000 & -6.10500000 & -4.05200000 \\
\hline$S$ & -4.10300000 & -7.12800000 & -1.52800000 & $\mathrm{H}$ & -6.05800000 & -5.13100000 & -3.93100000 \\
\hline $\mathrm{Fe}$ & e -6.08800000 & -6.38700000 & -0.50500000 & $\mathrm{H}$ & -5.32600000 & -6.25400000 & -5.10400000 \\
\hline 0 & -6.31300000 & -9.20000000 & -0.84500000 & $S$ & -3.95200000 & -5.89300000 & -3.17200000 \\
\hline \multirow[t]{2}{*}{0} & -6.20900000 & -8.22300000 & 0.03100000 & $\mathrm{~S}$ & -4.09900000 & -7.21100000 & -1.56900000 \\
\hline & & & & $\mathrm{Fe}$ & -6.25800000 & -6.51600000 & -0.57800000 \\
\hline \multicolumn{2}{|c|}{${ }^{5} \mathrm{R}$ for Model I } & & & 0 & -6.44300000 & -9.38000000 & -0.94700000 \\
\hline \multicolumn{2}{|c|}{-3328.151422 (a.u.) } & & & $\mathrm{O}$ & -6.43000000 & -8.45900000 & -0.00100000 \\
\hline C & -3.04900000 & -4.87800000 & 4.31400000 & & & & \\
\hline $\mathrm{H}$ & -3.63700000 & -5.51200000 & 4.97900000 & \multicolumn{2}{|c|}{${ }^{7} \mathrm{R}$ for model I } & & \\
\hline $\mathrm{H}$ & -3.21600000 & -3.84400000 & 4.64100000 & & 28.13342 (a.u.) & & \\
\hline$N$ & -3.12500000 & -4.36900000 & 1.79300000 & C & -3.08700000 & -4.90300000 & 4.32900000 \\
\hline $\mathrm{H}$ & -2.39700000 & -3.66000000 & 1.72800000 & $\mathrm{H}$ & -3.66400000 & -5.58100000 & 4.96000000 \\
\hline C & -3.52400000 & -5.09400000 & 2.91200000 & $\mathrm{H}$ & -3.27300000 & -3.89200000 & 4.71000000 \\
\hline C & -3.85400000 & -4.80400000 & 0.73900000 & $\mathrm{~N}$ & -3.19300000 & -4.23500000 & 1.84800000 \\
\hline $\mathrm{H}$ & -3.75000000 & -4.40600000 & -0.25800000 & $\mathrm{H}$ & -2.45400000 & -3.53300000 & 1.81200000 \\
\hline$N$ & -4.69900000 & -5.75800000 & 1.09900000 & C & -3.56000000 & -5.04900000 & 2.91600000 \\
\hline C & -4.49400000 & -5.95000000 & 2.44700000 & C & -3.90600000 & -4.62000000 & 0.76800000 \\
\hline $\mathrm{H}$ & -5.03300000 & -6.70400000 & 2.99700000 & $\mathrm{H}$ & -3.81100000 & -4.16900000 & -0.20600000 \\
\hline C & -9.83900000 & -3.60200000 & 2.65900000 & N & -4.71700000 & -5.62500000 & 1.06500000 \\
\hline $\mathrm{H}$ & -10.38800000 & -2.95000000 & 1.96700000 & C & -4.50300000 & -5.90300000 & 2.39800000 \\
\hline $\mathrm{H}$ & -9.04200000 & -2.99200000 & 3.09700000 & $\mathrm{H}$ & -5.02000000 & -6.70900000 & 2.89400000 \\
\hline$N$ & -9.86800000 & -5.88000000 & 1.47200000 & C & -9.80200000 & -3.39200000 & 2.72500000 \\
\hline $\mathrm{H}$ & -10.88800000 & -5.97900000 & 1.42100000 & $\mathrm{H}$ & -10.31800000 & -2.65000000 & 2.09900000 \\
\hline C & -9.19900000 & -4.76400000 & 1.95700000 & $\mathrm{H}$ & -8.97800000 & -2.86600000 & 3.21700000 \\
\hline C & -8.96000000 & -6.70500000 & 0.91800000 & N & -9.96600000 & -5.54100000 & 1.34100000 \\
\hline $\mathrm{H}$ & -9.21600000 & -7.63600000 & 0.44100000 & $\mathrm{H}$ & -10.99000000 & -5.62200000 & 1.34200000 \\
\hline$N$ & -7.73800000 & -6.19900000 & 1.01500000 & C & -9.22900000 & -4.51500000 & 1.91000000 \\
\hline C & -7.88000000 & -4.99400000 & 1.67400000 & C & -9.11500000 & -6.36800000 & 0.69700000 \\
\hline $\mathrm{H}$ & -7.02100000 & -4.38100000 & 1.89200000 & $\mathrm{H}$ & -9.43000000 & -7.24700000 & 0.15900000 \\
\hline C & -7.07100000 & -2.36100000 & -0.55600000 & N & -7.87100000 & -5.92200000 & 0.78400000 \\
\hline $\mathrm{H}$ & -6.78200000 & -2.55900000 & 0.47700000 & C & -7.93000000 & -4.78200000 & 1.55800000 \\
\hline H & -8.16100000 & -2.43600000 & -0.61500000 & $\mathrm{H}$ & -7.03500000 & -4.23500000 & 1.80400000 \\
\hline C & -6.51100000 & -3.45100000 & -1.49300000 & C & -7.11200000 & -2.31200000 & -0.62000000 \\
\hline $\mathrm{O}$ & -6.46300000 & -3.25900000 & -2.71400000 & $\mathrm{H}$ & -6.80800000 & -2.52600000 & 0.40400000 \\
\hline $\mathrm{O}$ & -6.18500000 & -4.53400000 & -0.87800000 & $\mathrm{H}$ & -8.20500000 & -2.35800000 & -0.66300000 \\
\hline C & -8.74700000 & -5.22000000 & -3.80000000 & C & -6.61100000 & -3.40700000 & -1.58900000 \\
\hline $\mathrm{H}$ & -8.73000000 & -4.16200000 & -3.51800000 & $\mathrm{O}$ & -6.69900000 & -3.21300000 & -2.81200000 \\
\hline
\end{tabular}




\begin{tabular}{|c|c|c|c|c|c|c|c|}
\hline $\mathrm{O}$ & -6.22400000 & -4.48100000 & -1.01100000 & $\mathrm{H}$ & -8.94200000 & -2.98000000 & 3.13000000 \\
\hline C & -9.08300000 & -5.44400000 & -2.97300000 & $\mathrm{~N}$ & -9.92500000 & -5.67000000 & 1.27000000 \\
\hline $\mathrm{H}$ & -9.45900000 & -4.94600000 & -2.06300000 & $\mathrm{H}$ & -10.95100000 & -5.73200000 & 1.25400000 \\
\hline $\mathrm{H}$ & -8.27200000 & -4.80500000 & -3.31800000 & $\mathrm{C}$ & -9.18700000 & -4.65900000 & 1.86500000 \\
\hline C & -8.62200000 & -6.79300000 & -2.39200000 & $\mathrm{C}$ & -9.07500000 & -6.50400000 & 0.63800000 \\
\hline $\mathrm{O}$ & -9.53400000 & -7.52700000 & -1.93700000 & $\mathrm{H}$ & -9.39000000 & -7.36400000 & 0.07200000 \\
\hline $\mathrm{N}$ & -7.30100000 & -7.16900000 & -2.22600000 & $\mathrm{~N}$ & -7.82300000 & -6.08200000 & 0.76500000 \\
\hline C & -6.38500000 & -7.38100000 & -3.36800000 & C & -7.88400000 & -4.94400000 & 1.54800000 \\
\hline $\mathrm{H}$ & -5.50900000 & -7.85300000 & -2.91300000 & $\mathrm{H}$ & -6.98700000 & -4.41400000 & 1.82100000 \\
\hline C & -5.87000000 & -6.09900000 & -4.07400000 & C & -7.10900000 & -2.38500000 & -0.60000000 \\
\hline $\mathrm{H}$ & -6.45500000 & -5.23100000 & -3.77400000 & $\mathrm{H}$ & -6.76300000 & -2.61600000 & 0.40800000 \\
\hline $\mathrm{H}$ & -5.83200000 & -6.10700000 & -5.17300000 & $\mathrm{H}$ & -8.20300000 & -2.43200000 & -0.60000000 \\
\hline$S$ & -4.12200000 & -5.63700000 & -3.58300000 & C & -6.64200000 & -3.45100000 & -1.61100000 \\
\hline$S$ & -3.88200000 & -6.61600000 & -1.76000000 & $\mathrm{O}$ & -6.79500000 & -3.24500000 & -2.82300000 \\
\hline $\mathrm{Fe}$ & -6.12500000 & -6.47800000 & -0.54300000 & $\mathrm{O}$ & -6.19100000 & -4.53500000 & -1.08400000 \\
\hline 0 & -5.58000000 & -8.53800000 & -0.28600000 & $\mathrm{~N}$ & -9.22200000 & -6.19000000 & -6.46400000 \\
\hline \multirow[t]{2}{*}{$\mathrm{O}$} & -6.12100000 & -8.13400000 & 0.83100000 & $\mathrm{H}$ & -8.35400000 & -5.64000000 & -6.60100000 \\
\hline & & & & $\mathrm{H}$ & -9.53100000 & -6.44500000 & -7.40900000 \\
\hline \multicolumn{2}{|c|}{${ }^{5} R$ for Model II } & & & $\mathrm{H}$ & -8.95800000 & -7.04700000 & -5.93700000 \\
\hline \multicolumn{2}{|c|}{-4312.115016 (a.u.) } & & & $C$ & -10.13500000 & -5.24000000 & -5.76200000 \\
\hline C & -3.07100000 & -4.92400000 & 4.28800000 & $\mathrm{H}$ & -11.11400000 & -5.29200000 & -6.23200000 \\
\hline $\mathrm{H}$ & -3.65800000 & -5.58400000 & 4.92900000 & $\mathrm{C}$ & -10.22300000 & -5.59700000 & -4.27500000 \\
\hline $\mathrm{H}$ & -3.25600000 & -3.90300000 & 4.64300000 & $\mathrm{H}$ & -10.70700000 & -6.56900000 & -4.14600000 \\
\hline$N$ & -3.18500000 & -4.28600000 & 1.80100000 & $\mathrm{H}$ & -10.89400000 & -4.85100000 & -3.84000000 \\
\hline H & -2.44900000 & -3.58100000 & 1.75800000 & C & -8.83900000 & -5.58500000 & -3.59700000 \\
\hline C & -3.54100000 & -5.09400000 & 2.87700000 & $\mathrm{H}$ & -8.73300000 & -4.70000000 & -2.96100000 \\
\hline C & -3.92900000 & -4.66200000 & 0.73600000 & $\mathrm{H}$ & -8.05300000 & -5.44000000 & -4.33300000 \\
\hline $\mathrm{H}$ & -3.85300000 & -4.20000000 & -0.23400000 & C & -8.52900000 & -6.77600000 & -2.69300000 \\
\hline$N$ & -4.74600000 & -5.65800000 & 1.04800000 & $\mathrm{O}$ & -9.49400000 & -7.31300000 & -2.10600000 \\
\hline C & -4.50300000 & -5.93900000 & 2.37600000 & C & -9.55300000 & -3.80300000 & -5.95900000 \\
\hline $\mathrm{H}$ & -5.01200000 & -6.74200000 & 2.88500000 & $\mathrm{O}$ & -8.34500000 & -3.75100000 & -6.33900000 \\
\hline C & -1.71000000 & -10.04100000 & -0.48400000 & 0 & -10.34900000 & -2.88700000 & -5.68100000 \\
\hline $\mathrm{H}$ & -1.14200000 & -10.97400000 & -0.50700000 & $\mathrm{~N}$ & & & -2.42700000 \\
\hline $\mathrm{H}$ & -2.30200000 & -9.99600000 & -1.40500000 & C & -6.32000000 & -7.32100000 & -3.56700000 \\
\hline$N$ & -0.99100000 & -7.57700000 & -0.52200000 & $\mathrm{H}$ & -5.52700000 & -7.96600000 & -3.17600000 \\
\hline $\mathrm{H}$ & -1.90300000 & -7.18000000 & -0.75100000 & C & -5.62100000 & -6.05600000 & -4.12500000 \\
\hline C & -0.70900000 & -8.92700000 & -0.39100000 & $\mathrm{H}$ & -6.19100000 & -5.15900000 & -3.89100000 \\
\hline C & 0.16000000 & -6.88900000 & -0.31400000 & $\mathrm{H}$ & -5.47100000 & -6.12800000 & -5.20500000 \\
\hline $\mathrm{H}$ & 0.21600000 & -5.80700000 & -0.35400000 & $S$ & -3.93900000 & -5.74200000 & -3.38600000 \\
\hline$N$ & 1.17300000 & -7.70200000 & -0.06700000 & $S$ & -3.97800000 & -6.93500000 & -1.67900000 \\
\hline C & 0.64000000 & -8.97400000 & -0.12000000 & $\mathrm{C}$ & -6.94500000 & -8.12700000 & -4.71200000 \\
\hline $\mathrm{H}$ & 1.24200000 & -9.85700000 & 0.04200000 & 0 & -8.18400000 & -8.18600000 & -4.89100000 \\
\hline C & -9.76600000 & -3.52000000 & 2.65200000 & $\mathrm{~N}$ & -6.11100000 & -8.77200000 & -5.53200000 \\
\hline $\mathrm{H}-$ & -10.28200000 & -2.79800000 & 2.00600000 & $\mathrm{H}$ & -5.10000000 & -8.72400000 & -5.38400000 \\
\hline
\end{tabular}




\begin{tabular}{|c|c|c|c|c|c|c|c|}
\hline C & -6.64300000 & -9.50300000 & -6.67300000 & $\mathrm{H}$ & -7.00900000 & -4.64300000 & 1.92500000 \\
\hline $\mathrm{H}$ & -7.29000000 & -10.31500000 & -6.33000000 & C & -7.13500000 & -2.39200000 & -0.57200000 \\
\hline H & -5.80300000 & -9.95100000 & -7.20700000 & $\mathrm{H}$ & -6.79900000 & -2.61900000 & 0.44100000 \\
\hline C & -7.44600000 & -8.62300000 & -7.66300000 & $\mathrm{H}$ & -8.23000000 & -2.43300000 & -0.58000000 \\
\hline O & -8.31300000 & -9.25100000 & -8.35400000 & C & -6.66800000 & -3.47700000 & -1.56700000 \\
\hline 0 & -7.18600000 & -7.40200000 & -7.73200000 & 0 & -6.76800000 & -3.26600000 & -2.78700000 \\
\hline $\mathrm{Fe}$ & -6.18600000 & -6.50400000 & -0.68000000 & $\mathrm{O}$ & -6.27400000 & -4.56800000 & -1.02300000 \\
\hline 0 & -5.89700000 & -9.35000000 & -0.65100000 & $\mathrm{~N}$ & -9.23400000 & -6.15800000 & -6.52600000 \\
\hline \multirow[t]{2}{*}{$\mathrm{O}$} & -6.00000000 & -8.31900000 & 0.16200000 & $\mathrm{H}$ & -8.36000000 & -5.61600000 & -6.65400000 \\
\hline & & & & $\mathrm{H}$ & -9.53700000 & -6.41100000 & -7.47300000 \\
\hline \multicolumn{2}{|c|}{${ }^{5}$ TS1a } & & & $\mathrm{H}$ & -8.98400000 & -7.01900000 & -5.99700000 \\
\hline \multicolumn{2}{|c|}{-4312.094827 (a.u.) } & & & C - & -10.14400000 & -5.19700000 & -5.83200000 \\
\hline C & -3.01700000 & -4.94100000 & 4.37200000 & $\mathrm{H}$ & -11.11600000 & -5.23200000 & -6.32000000 \\
\hline $\mathrm{H}$ & -3.59000000 & -5.59500000 & 5.02900000 & C - & -10.25800000 & -5.55900000 & -4.34800000 \\
\hline $\mathrm{H}$ & -3.19400000 & -3.91800000 & 4.72400000 & $\mathrm{H}$ & -10.76100000 & -6.52200000 & -4.23000000 \\
\hline $\mathrm{N}$ & -3.21700000 & -4.28700000 & 1.89900000 & $\mathrm{H}$ & -10.91900000 & -4.80300000 & -3.91600000 \\
\hline $\mathrm{H}$ & -2.50400000 & -3.56100000 & 1.84300000 & C & -8.88100000 & -5.58200000 & -3.65300000 \\
\hline C & -3.51400000 & -5.11800000 & 2.97200000 & $\mathrm{H}$ & -8.76800000 & -4.70300000 & -3.00900000 \\
\hline C & -3.97800000 & -4.68300000 & 0.85000000 & $\mathrm{H}$ & -8.08000000 & -5.44500000 & -4.37800000 \\
\hline $\mathrm{H}$ & -3.96500000 & -4.18900000 & -0.10900000 & $\mathrm{C}$ & -8.61100000 & -6.78700000 & -2.75000000 \\
\hline $\mathrm{N}$ & -4.74500000 & -5.71400000 & 1.16900000 & 0 & -9.60000000 & -7.28700000 & -2.15300000 \\
\hline C & -4.45400000 & -5.99600000 & 2.48500000 & C & -9.53900000 & -3.76900000 & -6.01000000 \\
\hline $\mathrm{H}$ & -4.91600000 & -6.82200000 & 3.00200000 & 0 & -8.33000000 & -3.72800000 & -6.38800000 \\
\hline C & -1.64200000 & -10.10500000 & -0.39000000 & 0 & -10.32300000 & -2.84500000 & -5.72000000 \\
\hline $\mathrm{H}$ & -1.05400000 & -11.02500000 & -0.39200000 & $\mathrm{~N}$ & -7.34300000 & -7.19600000 & -2.48900000 \\
\hline $\mathrm{H}$ & -2.23900000 & -10.09500000 & -1.30800000 & C & -6.39900000 & -7.34300000 & -3.58400000 \\
\hline $\mathrm{N}$ & -0.99800000 & -7.62400000 & -0.45700000 & $\mathrm{H}$ & -5.63200000 & -8.01900000 & -3.19600000 \\
\hline $\mathrm{H}$ & -1.92400000 & -7.25300000 & -0.65000000 & C & -5.65600000 & -6.06100000 & -4.08500000 \\
\hline C & -0.67200000 & -8.96400000 & -0.32800000 & $\mathrm{H}$ & -6.13200000 & -5.15500000 & -3.70500000 \\
\hline C & 0.13800000 & -6.89800000 & -0.28900000 & $\mathrm{H}$ & -5.59100000 & -6.01300000 & -5.17600000 \\
\hline H & 0.16000000 & -5.81600000 & -0.33500000 & $S$ & -3.89900000 & -5.99200000 & -3.50600000 \\
\hline $\mathrm{N}$ & 1.18000000 & -7.68000000 & -0.07200000 & $S$ & -4.03300000 & -6.83500000 & -1.64500000 \\
\hline C & 0.68500000 & -8.96700000 & -0.10100000 & $\mathrm{C}$ & -6.98100000 & -8.11600000 & -4.78300000 \\
\hline $\mathrm{H}$ & 1.31700000 & -9.83200000 & 0.04600000 & $\mathrm{O}$ & -8.21000000 & -8.19100000 & -5.00300000 \\
\hline C & -9.76800000 & -3.59400000 & 2.64400000 & $\mathrm{~N}$ & -6.11700000 & -8.73100000 & -5.59600000 \\
\hline $\mathrm{H}$ & -10.28000000 & -2.87500000 & 1.99100000 & $\mathrm{H}$ & -5.11000000 & -8.68600000 & -5.42600000 \\
\hline H & -8.93100000 & -3.06100000 & 3.10500000 & $\mathrm{C}$ & -6.62200000 & -9.47100000 & -6.74400000 \\
\hline $\mathrm{N}$ & -9.99000000 & -5.72200000 & 1.24000000 & $\mathrm{H}$ & -7.24500000 & -10.30500000 & -6.40900000 \\
\hline $\mathrm{H}$ & -11.01700000 & -5.73100000 & 1.19800000 & $\mathrm{H}$ & -5.76700000 & -9.89000000 & -7.28000000 \\
\hline C & -9.21900000 & -4.75900000 & 1.87400000 & $\mathrm{C}$ & -7.45000000 & -8.60900000 & -7.72900000 \\
\hline C & -9.16600000 & -6.60400000 & 0.64300000 & $\mathrm{O}$ & -8.31300000 & -9.25300000 & -8.41000000 \\
\hline $\mathrm{H}$ & -9.50300000 & -7.43000000 & 0.03800000 & 0 & -7.20700000 & -7.38400000 & -7.80900000 \\
\hline $\mathrm{N}$ & -7.89700000 & -6.26500000 & 0.83600000 & $\mathrm{Fe}$ & -6.30600000 & -6.56000000 & -0.59300000 \\
\hline C & -7.92400000 & -5.12400000 & 1.61900000 & 0 & -4.68500000 & -8.79200000 & -1.20300000 \\
\hline
\end{tabular}




\begin{tabular}{|c|c|c|c|c|c|c|c|}
\hline 0 & -5.70400000 & -8.48800000 & -0.33300000 & $\mathrm{H}$ & -8.36100000 & -5.70100000 & -6.61800000 \\
\hline & & & & $\mathrm{H}$ & -9.54100000 & -6.51800000 & -7.40900000 \\
\hline & n1a & & & $\mathrm{H}$ & -8.97100000 & -7.08800000 & -5.92500000 \\
\hline & 312.153782 (a.u.) & & & C - & -10.15100000 & -5.28000000 & -5.79000000 \\
\hline C & -3.06500000 & -4.91700000 & 4.32600000 & $\mathrm{H}-$ & -11.12300000 & -5.33100000 & -6.27500000 \\
\hline $\mathrm{H}$ & -3.64600000 & -5.59200000 & 4.95600000 & C - & -10.26000000 & -5.62700000 & -4.30300000 \\
\hline $\mathrm{H}$ & -3.24700000 & -3.90500000 & 4.70900000 & $\mathrm{H}$ - & -10.74900000 & -6.59700000 & -4.17400000 \\
\hline $\mathrm{N}$ & -3.24500000 & -4.17700000 & 1.87600000 & $\mathrm{H}-$ & -10.93400000 & -4.87700000 & -3.87900000 \\
\hline $\mathrm{H}$ & -2.49700000 & -3.48600000 & 1.83400000 & C & -8.88100000 & -5.61300000 & -3.61600000 \\
\hline C & -3.55200000 & -5.04700000 & 2.91700000 & $\mathrm{H}$ & -8.77700000 & -4.71700000 & -2.99500000 \\
\hline C & -4.01900000 & -4.51200000 & 0.81700000 & $\mathrm{H}$ & -8.09000000 & -5.48400000 & -4.34900000 \\
\hline $\mathrm{H}$ & -3.98500000 & -3.99500000 & -0.12800000 & $\mathrm{C}$ & -8.58000000 & -6.78500000 & -2.68400000 \\
\hline $\mathrm{N}$ & -4.80600000 & -5.54000000 & 1.10300000 & $\mathrm{O}$ & -9.55600000 & -7.29500000 & -2.08200000 \\
\hline C & -4.50900000 & -5.88800000 & 2.40200000 & $\mathrm{C}$ & -9.56200000 & -3.84600000 & -5.98400000 \\
\hline $\mathrm{H}$ & -4.98600000 & -6.72800000 & 2.88300000 & 0 & -8.35000000 & -3.79500000 & -6.35300000 \\
\hline C & -1.45300000 & -10.42000000 & -0.43500000 & 0 & -10.35800000 & -2.92600000 & -5.71400000 \\
\hline $\mathrm{H}$ & -0.85700000 & -11.29300000 & -0.16400000 & $\mathrm{~N}$ & -7.29400000 & -7.14700000 & -2.40100000 \\
\hline $\mathrm{H}$ & -1.94900000 & -10.66000000 & -1.38100000 & $\mathrm{C}$ & -6.34400000 & -7.35000000 & -3.49100000 \\
\hline $\mathrm{N}$ & -0.89500000 & -8.14200000 & -1.33500000 & $\mathrm{H}$ & -5.55500000 & -7.99000000 & -3.08600000 \\
\hline $\mathrm{H}$ & -1.86000000 & -8.02300000 & -1.66600000 & C & -5.66400000 & -6.07500000 & -4.07700000 \\
\hline C & -0.52200000 & -9.26600000 & -0.62800000 & $\mathrm{H}$ & -6.24900000 & -5.18700000 & -3.84600000 \\
\hline C & 0.16600000 & -7.29100000 & -1.34800000 & $\mathrm{H}$ & -5.52700000 & -6.15600000 & -5.15900000 \\
\hline $\mathrm{H}$ & 0.13300000 & -6.31600000 & -1.81600000 & S & -3.98800000 & -5.72900000 & -3.37900000 \\
\hline $\mathrm{N}$ & 1.20400000 & -7.80400000 & -0.71400000 & $S$ & -3.97300000 & -6.74800000 & -1.50900000 \\
\hline C & 0.78400000 & -9.03800000 & -0.26300000 & C & -6.95100000 & -8.16700000 & -4.65300000 \\
\hline $\mathrm{H}$ & 1.43500000 & -9.69600000 & 0.29600000 & $\mathrm{O}$ & -8.18600000 & -8.21800000 & -4.85000000 \\
\hline C & -9.78000000 & -3.45000000 & 2.70100000 & $\mathrm{~N}$ & -6.11500000 & -8.80600000 & -5.47500000 \\
\hline $\mathrm{H}$ & -10.29700000 & -2.71300000 & 2.07200000 & $\mathrm{H}$ & -5.10000000 & -8.75400000 & -5.35200000 \\
\hline $\mathrm{H}$ & -8.95000000 & -2.92500000 & 3.18400000 & C & -6.64500000 & -9.52000000 & -6.62800000 \\
\hline $\mathrm{N}$ & -9.97100000 & -5.56900000 & 1.27700000 & $\mathrm{H}$ & -7.30500000 & -10.32700000 & -6.30000000 \\
\hline $\mathrm{H}$ & -10.99800000 & -5.62700000 & 1.26800000 & $\mathrm{H}$ & -5.80200000 & -9.97100000 & -7.15700000 \\
\hline C & -9.21700000 & -4.57800000 & 1.88600000 & C & -7.42500000 & -8.62100000 & -7.61900000 \\
\hline C & -9.14000000 & -6.40300000 & 0.62400000 & $\mathrm{O}$ & -8.30600000 & -9.22600000 & -8.31400000 \\
\hline $\mathrm{H}$ & -9.46800000 & -7.24400000 & 0.03800000 & $\mathrm{O}$ & -7.13400000 & -7.40700000 & -7.68500000 \\
\hline $\mathrm{N}$ & -7.88400000 & -5.99400000 & 0.75100000 & $\mathrm{Fe}$ & -6.28300000 & -6.53800000 & -0.56200000 \\
\hline C & -7.92000000 & -4.87100000 & 1.55500000 & $\mathrm{O}$ & -3.61500000 & -8.24000000 & -1.79400000 \\
\hline $\mathrm{H}$ & -7.01300000 & -4.36200000 & 1.83400000 & $\mathrm{O}$ & -6.07900000 & -8.05600000 & 0.01200000 \\
\hline C & -7.08500000 & -2.42600000 & -0.59500000 & & & & \\
\hline $\mathrm{H}$ & -6.71200000 & -2.64500000 & 0.40500000 & ${ }^{5} \mathrm{TS}$ & $2 a$ & & \\
\hline $\mathrm{H}$ & -8.17900000 & -2.47000000 & -0.56100000 & & 312.140869 (a.u.) & & \\
\hline C & -6.66300000 & -3.51500000 & -1.60400000 & $\mathrm{C}$ & -3.01500000 & -4.84500000 & 4.31700000 \\
\hline 0 & -6.83300000 & -3.31400000 & -2.81400000 & $\mathrm{H}$ & -3.61600000 & -5.51600000 & 4.93400000 \\
\hline 0 & -6.22700000 & -4.60200000 & -1.07200000 & $\mathrm{H}$ & -3.18200000 & -3.83400000 & 4.70900000 \\
\hline $\mathrm{N}$ & -9.23300000 & -6.24200000 & -6.47100000 & $\mathrm{~N}$ & -3.13100000 & -4.13200000 & 1.84100000 \\
\hline
\end{tabular}




\begin{tabular}{|c|c|c|c|c|c|c|c|}
\hline 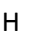 & -2.36300000 & -3.46000000 & 1.79100000 & C & -8.85300000 & -5.60900000 & -3.63200000 \\
\hline r & -3.48300000 & -4.96200000 & 2.90000000 & $\mathrm{H}$ & -8.72700000 & -4.73500000 & -2.98500000 \\
\hline & -3.90400000 & -4.47900000 & 0.77800000 & $\mathrm{H}$ & -8.04600000 & -5.48700000 & -4.35300000 \\
\hline & -3.83100000 & -3.99600000 & -0.18500000 & C & -8.61100000 & -6.81800000 & -2.73600000 \\
\hline & -4.72700000 & -5.47000000 & 1.07900000 & 0 & -9.60000000 & -7.31200000 & -2.14700000 \\
\hline & -4.46100000 & -5.78300000 & 2.39200000 & C & -9.53100000 & -3.77400000 & -5.98400000 \\
\hline & -4.96900000 & -6.59300000 & 2.89200000 & $\mathrm{O}$ & -8.32100000 & -3.72700000 & -6.35800000 \\
\hline & -1.47300000 & -10.36300000 & -0.44200000 & 0 & -10.32200000 & -2.85500000 & -5.69900000 \\
\hline & -0.83100000 & -11.20700000 & -0.18700000 & $\mathrm{~N}$ & -7.34600000 & -7.24700000 & -2.44200000 \\
\hline & -1.98400000 & -10.62400000 & -1.37400000 & $\mathrm{C}$ & -6.35400000 & -7.44000000 & -3.49600000 \\
\hline & -0.99700000 & -8.07700000 & -1.40800000 & $\mathrm{H}$ & -5.65500000 & -8.18900000 & -3.11300000 \\
\hline & -1.91900000 & -7.98900000 & -1.81500000 & $\mathrm{C}$ & -5.50600000 & -6.19200000 & -3.90500000 \\
\hline & -0.60100000 & -9.16900000 & -0.65700000 & $\mathrm{H}$ & -6.07300000 & -5.27400000 & -3.75400000 \\
\hline & 0.02900000 & -7.18200000 & -1.42500000 & $\mathrm{H}$ & -5.20900000 & -6.27100000 & -4.95200000 \\
\hline & -0.03400000 & -6.22500000 & -1.92600000 & $\mathrm{~S}$ & -3.92900000 & -5.94900000 & -2.95000000 \\
\hline & 1.06500000 & -7.63500000 & -0.74900000 & $S$ & -4.23200000 & -7.31100000 & -1.16200000 \\
\hline & 0.68500000 & -8.87300000 & -0.27300000 & C & -6.96100000 & -8.13100000 & -4.73900000 \\
\hline & 1.35000000 & -9.48700000 & 0.31700000 & 0 & -8.19600000 & -8.17800000 & -4.93200000 \\
\hline & -9.82000000 & -3.47700000 & 2.71100000 & $\mathrm{~N}$ & -6.12800000 & -8.72100000 & -5.60000000 \\
\hline & -10.33900000 & -2.73200000 & 2.09400000 & $\mathrm{H}$ & -5.11400000 & -8.70200000 & -5.46500000 \\
\hline & -8.97200000 & -2.96600000 & 3.17600000 & C & -6.66800000 & -9.46700000 & -6.72900000 \\
\hline & -10.09000000 & -5.58900000 & 1.29600000 & $\mathrm{H}$ & -7.30300000 & -10.28000000 & -6.36700000 \\
\hline & -11.11800000 & -5.61600000 & 1.28700000 & $\mathrm{H}$ & -5.82700000 & -9.91500000 & -7.26300000 \\
\hline & -9.29900000 & -4.62000000 & 1.89300000 & C & -7.48600000 & -8.61500000 & -7.72600000 \\
\hline & -9.29500000 & -6.45800000 & 0.64800000 & $\mathrm{O}$ & -8.34100000 & -9.26900000 & -8.40800000 \\
\hline & -9.65000000 & -7.29400000 & 0.06800000 & $\mathrm{O}$ & -7.24800000 & -7.39100000 & -7.81600000 \\
\hline & -8.02400000 & -6.09600000 & 0.77100000 & $\mathrm{Fe}$ & -6.49700000 & -6.65000000 & -0.60700000 \\
\hline & -8.01500000 & -4.96200000 & 1.56000000 & $\mathrm{O}$ & -3.95600000 & -8.67400000 & -1.80700000 \\
\hline & -7.08700000 & -4.48200000 & 1.82000000 & 0 & -5.87500000 & -8.15900000 & -0.01800000 \\
\hline & -7.08300000 & -2.45800000 & -0.58600000 & & & & \\
\hline & -6.70500000 & -2.68500000 & 0.41200000 & \multicolumn{2}{|c|}{${ }^{5} \mathrm{IM} 2 \mathrm{a}$} & & \\
\hline & -8.17700000 & -2.50500000 & -0.53900000 & \multicolumn{2}{|c|}{-4312.219502 (a.u.) } & & \\
\hline & -6.67200000 & -3.55400000 & -1.59500000 & C & -3.11800000 & -4.89500000 & 4.17000000 \\
\hline & -6.71500000 & -3.31600000 & -2.81000000 & $\mathrm{H}$ & -3.72900000 & -5.56600000 & 4.77700000 \\
\hline & -6.40600000 & -4.69100000 & -1.06000000 & $\mathrm{H}$ & -3.32800000 & -3.87800000 & 4.52500000 \\
\hline & -9.21700000 & -6.16000000 & -6.50300000 & $\mathrm{~N}$ & -3.13700000 & -4.25400000 & 1.67000000 \\
\hline & -8.33800000 & -5.62300000 & -6.61900000 & $\mathrm{H}$ & -2.38400000 & -3.56300000 & 1.64200000 \\
\hline & -9.51000000 & -6.39600000 & -7.45800000 & C & -3.53100000 & -5.05400000 & 2.73800000 \\
\hline & -8.97500000 & -7.02800000 & -5.98700000 & C & -3.87300000 & -4.61500000 & 0.59100000 \\
\hline & -10.12700000 & -5.20500000 & -5.80500000 & $\mathrm{H}$ & -3.77700000 & -4.14900000 & -0.37800000 \\
\hline & -11.10300000 & -5.24600000 & -6.28400000 & $\mathrm{~N}$ & -4.71800000 & -5.59200000 & 0.88500000 \\
\hline & -10.23200000 & -5.57200000 & -4.32300000 & C & -4.50100000 & -5.87600000 & 2.21700000 \\
\hline & -10.74500000 & -6.53000000 & -4.20800000 & $\mathrm{H}$ & -5.03800000 & -6.66400000 & 2.72200000 \\
\hline & -10.88300000 & -4.81100000 & -3.88200000 & C & -1.14400000 & -10.52500000 & -0.23600000 \\
\hline
\end{tabular}




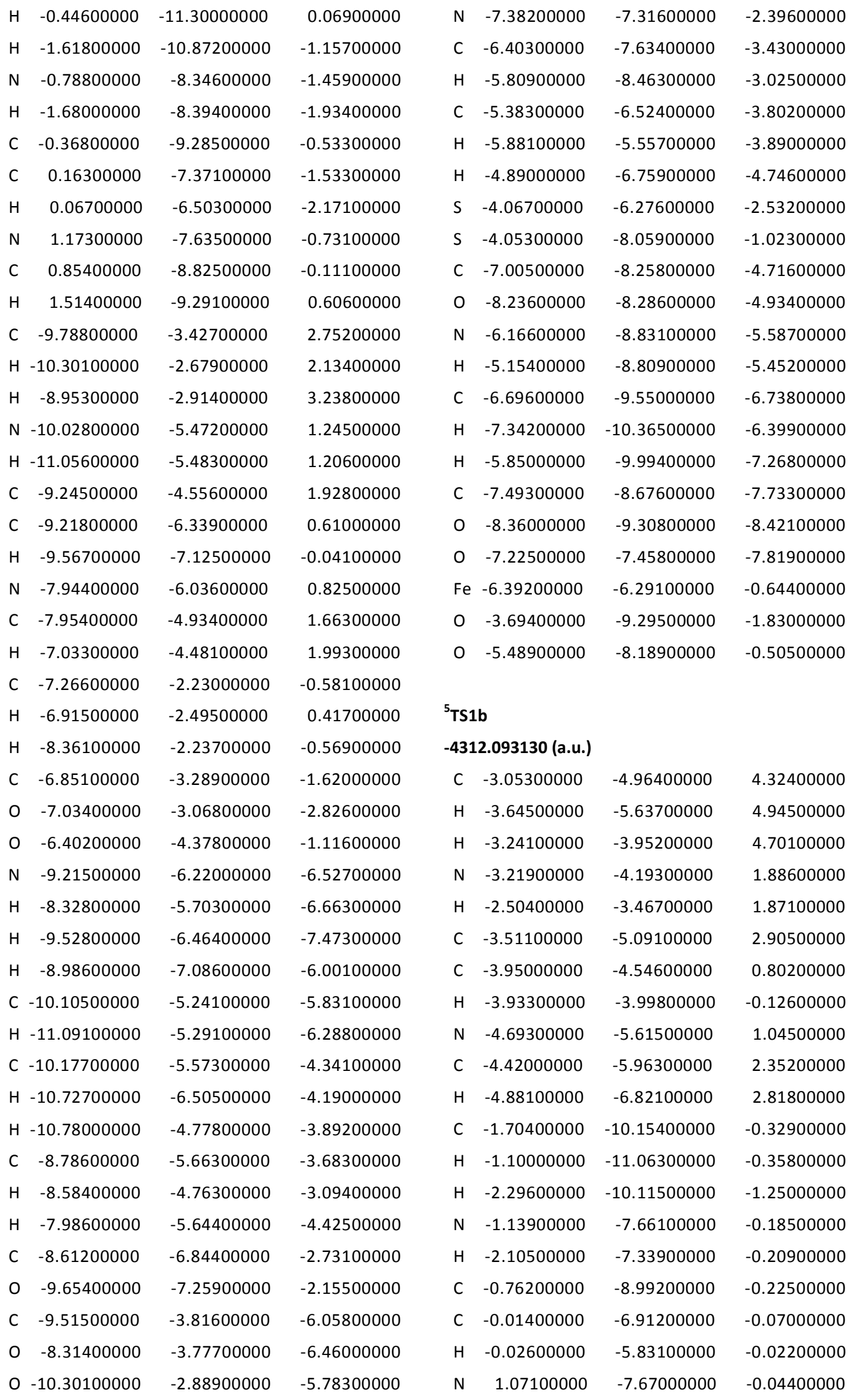




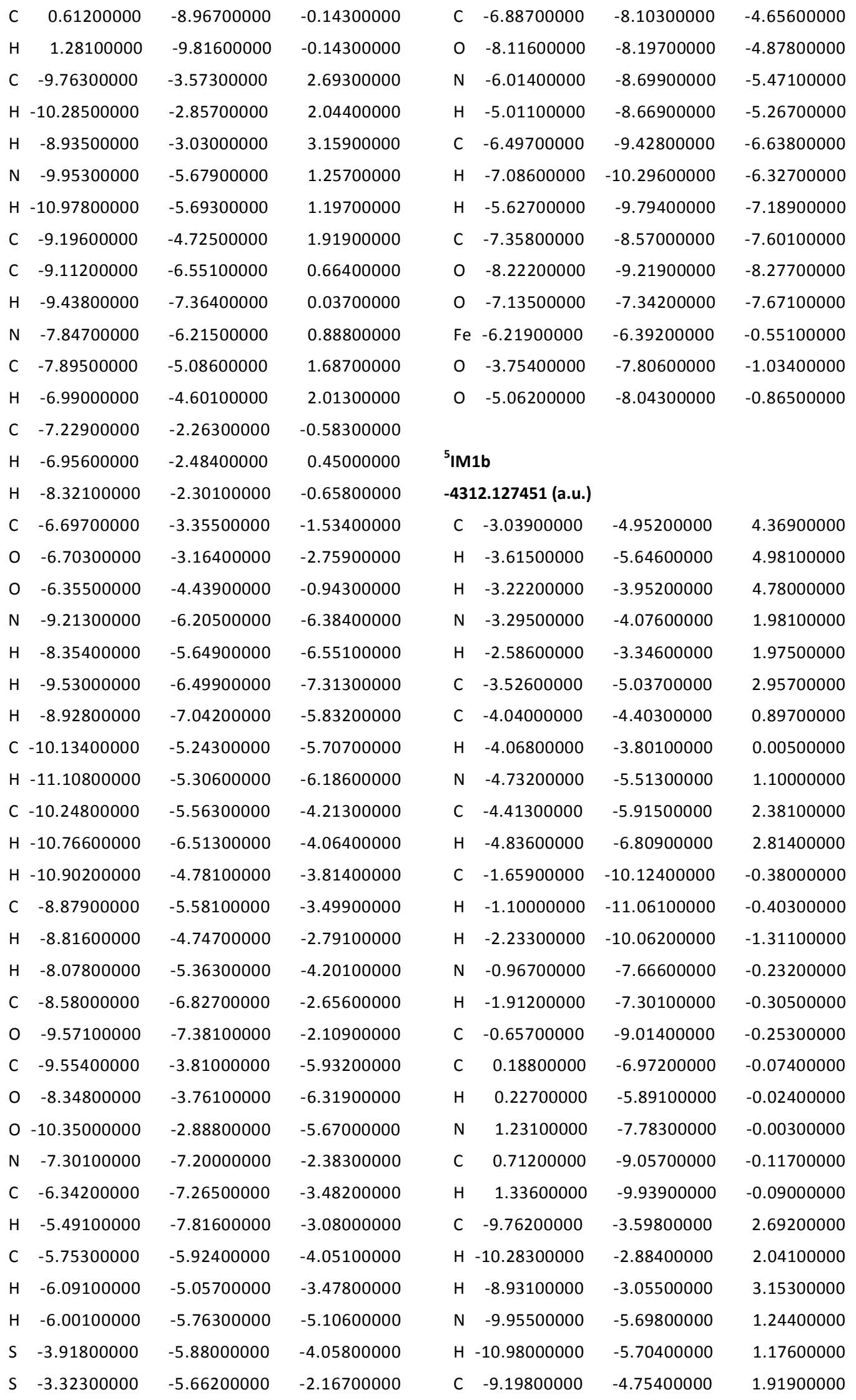




\begin{tabular}{|c|c|c|c|c|c|c|c|}
\hline & -9.11400000 & -6.57200000 & 0.65300000 & 0 & -8.24100000 & -9.24400000 & -8.32800000 \\
\hline $\mathrm{H}$ & -9.44000000 & -7.37500000 & 0.01400000 & 0 & -7.16100000 & -7.36700000 & -7.70900000 \\
\hline & -7.84900000 & -6.24600000 & 0.89100000 & $\mathrm{~F}$ & -6.24800000 & -6.38800000 & -0.58600000 \\
\hline & -7.89800000 & -5.12200000 & 1.69600000 & $c$ & -3.54300000 & -7.30500000 & -1.25400000 \\
\hline & -6.99300000 & -4.64100000 & 2.03000000 & c & -4.89500000 & -7.82300000 & -0.96600000 \\
\hline & -7.26100000 & -2.22800000 & -0.60400000 & & & & \\
\hline & -7.00800000 & -2.44600000 & 0.43500000 & \multicolumn{4}{|c|}{${ }^{5}$ TS2b } \\
\hline & -8.35300000 & -2.25800000 & -0.69400000 & \multicolumn{4}{|c|}{-4312.044769 (a.u.) } \\
\hline & -6.72900000 & -3.33900000 & -1.53200000 & C & -3.06800000 & -4.94800000 & 4.29500000 \\
\hline & -6.65800000 & -3.15500000 & -2.75600000 & $\mathrm{H}$ & -3.66300000 & -5.64300000 & 4.88900000 \\
\hline & -6.47200000 & -4.43100000 & -0.91600000 & $\mathrm{H}$ & -3.26000000 & -3.95000000 & 4.70800000 \\
\hline & -9.22400000 & -6.20400000 & -6.41900000 & $\mathrm{~N}$ & -3.23400000 & -4.10300000 & 1.87200000 \\
\hline - & -8.35900000 & -5.65300000 & -6.57300000 & $\mathrm{H}$ & -2.47400000 & -3.42300000 & 1.84600000 \\
\hline & -9.53400000 & -6.48600000 & -7.35400000 & C & -3.53800000 & -5.02000000 & 2.87400000 \\
\hline & -8.95100000 & -7.04900000 & -5.87300000 & C & -4.03600000 & -4.36800000 & 0.81200000 \\
\hline & -10.14300000 & -5.24000000 & -5.74200000 & $\mathrm{H}$ & -4.00800000 & -3.80800000 & -0.10800000 \\
\hline-1 & -11.11400000 & -5.29300000 & -6.22900000 & $\mathrm{~N}$ & -4.83500000 & -5.39400000 & 1.05600000 \\
\hline & -10.26900000 & -5.57000000 & -4.25200000 & C & -4.52500000 & -5.80900000 & 2.33400000 \\
\hline$H$ & -10.79300000 & -6.51900000 & -4.11400000 & $\mathrm{H}$ & -5.01500000 & -6.65400000 & 2.79300000 \\
\hline$H$ & -10.91900000 & -4.78900000 & -3.84800000 & C & -1.55500000 & -10.08600000 & -0.29900000 \\
\hline C & -8.90300000 & -5.60500000 & -3.53400000 & $\mathrm{H}$ & -1.01800000 & -11.03200000 & -0.38500000 \\
\hline - & -8.82600000 & -4.76700000 & -2.83100000 & $\mathrm{H}$ & -2.12500000 & -9.94700000 & -1.22300000 \\
\hline H & -8.09500000 & -5.40900000 & -4.23600000 & $\mathrm{~N}$ & -0.76000000 & -7.67400000 & 0.12000000 \\
\hline & -8.62600000 & -6.85200000 & -2.68300000 & $\mathrm{H}$ & -1.68000000 & -7.27400000 & 0.23900000 \\
\hline 0 & -9.62900000 & -7.38300000 & -2.13100000 & C & -0.51600000 & -9.01600000 & -0.11400000 \\
\hline$r$ & -9.55300000 & -3.80800000 & -5.95200000 & C & 0.43200000 & -7.03500000 & 0.20600000 \\
\hline 0 & -8.34600000 & -3.76400000 & -6.33500000 & $\mathrm{H}$ & 0.52200000 & -5.96900000 & 0.37800000 \\
\hline $\mathrm{O}$ & -10.34500000 & -2.88400000 & -5.68500000 & $\mathrm{~N}$ & 1.44100000 & -7.87500000 & 0.03600000 \\
\hline $\mathrm{N}$ & -7.35700000 & -7.24700000 & -2.41100000 & C & 0.85600000 & -9.10900000 & -0.16400000 \\
\hline$r$ & -6.39200000 & -7.31800000 & -3.50600000 & $\pi$ & 1.43700000 & -10.00300000 & -0.33300000 \\
\hline $\mathrm{H}$ & -5.56500000 & -7.89100000 & -3.09100000 & C & -9.77000000 & -3.51100000 & 2.70400000 \\
\hline$c$ & -5.75500000 & -5.99000000 & -4.03900000 & $\mathrm{H}$ & -10.29300000 & -2.77900000 & 2.07400000 \\
\hline $\mathrm{H}$ & -6.12500000 & -5.11100000 & -3.50700000 & $\mathrm{H}$ & -8.94100000 & -2.98000000 & 3.18100000 \\
\hline $\mathrm{H}$ & -5.92100000 & -5.83500000 & -5.11200000 & $\mathrm{~N}$ & -9.95600000 & -5.58800000 & 1.22300000 \\
\hline & -3.91400000 & -5.94900000 & -3.90500000 & $\mathrm{H}$ & -10.98300000 & -5.62000000 & 1.18000000 \\
\hline & -3.54000000 & -5.75000000 & -1.90000000 & C & -9.20400000 & -4.64000000 & 1.89600000 \\
\hline$c$ & -6.93500000 & -8.13700000 & -4.69100000 & $c$ & -9.11400000 & -6.42900000 & 0.59000000 \\
\hline $\mathrm{O}$ & -8.16200000 & -8.22800000 & -4.92800000 & $\mathrm{H}$ & -9.43700000 & -7.22800000 & -0.05600000 \\
\hline $\mathrm{N}$ & -6.05700000 & -8.73400000 & -5.50200000 & $\mathrm{~N}$ & -7.85000000 & -6.07600000 & 0.79800000 \\
\hline $\mathrm{H}$ & -5.05400000 & -8.70400000 & -5.30000000 & C & -7.90200000 & -4.97000000 & 1.63100000 \\
\hline C & -6.53400000 & -9.45700000 & -6.67400000 & $\mathrm{H}$ & -6.99900000 & -4.48200000 & 1.95700000 \\
\hline $\mathrm{H}$ & -7.12900000 & -10.32300000 & -6.37000000 & C & -7.27600000 & -2.20900000 & -0.61400000 \\
\hline $\mathrm{H}$ & -5.66000000 & -9.82500000 & -7.21700000 & $\mathrm{H}$ & -6.95000000 & -2.47600000 & 0.39200000 \\
\hline & -7.38400000 & -8.59600000 & -7.64300000 & $\mathrm{H}$ & -8.37100000 & -2.22800000 & -0.63900000 \\
\hline
\end{tabular}




\begin{tabular}{|c|c|c|c|}
\hline C & -6.80200000 & -3.23700000 & -1.65700000 \\
\hline 0 & -7.01900000 & -3.03200000 & -2.85600000 \\
\hline 0 & -6.24300000 & -4.29000000 & -1.17100000 \\
\hline $\mathrm{N}$ & -9.24600000 & -6.17300000 & -6.45200000 \\
\hline $\mathrm{H}$ & -8.38600000 & -5.61200000 & -6.59900000 \\
\hline $\mathrm{H}$ & -9.55100000 & -6.44500000 & -7.39200000 \\
\hline $\mathrm{H}$ & -8.96900000 & -7.02200000 & -5.91500000 \\
\hline$C$ & -10.16800000 & -5.22400000 & -5.76200000 \\
\hline $\mathrm{H}$ & -11.14200000 & -5.28000000 & -6.24300000 \\
\hline$C$ & -10.28300000 & -5.57500000 & -4.27500000 \\
\hline $\mathrm{H}$ & -10.78800000 & -6.53600000 & -4.15100000 \\
\hline $\mathrm{H}$ & -10.95000000 & -4.81500000 & -3.85700000 \\
\hline$C$ & -8.91700000 & -5.58700000 & -3.55700000 \\
\hline $\mathrm{H}$ & -8.84300000 & -4.72600000 & -2.88400000 \\
\hline $\mathrm{H}$ & -8.10800000 & -5.40700000 & -4.26100000 \\
\hline$C$ & -8.63200000 & -6.81000000 & -2.67600000 \\
\hline 0 & -9.63100000 & -7.34800000 & -2.12500000 \\
\hline$C$ & -9.59200000 & -3.78600000 & -5.96600000 \\
\hline 0 & -8.39100000 & -3.73100000 & -6.36900000 \\
\hline 0 & -10.38700000 & -2.87000000 & -5.68400000 \\
\hline $\mathrm{N}$ & -7.35400000 & -7.18400000 & -2.38900000 \\
\hline$C$ & -6.41100000 & -7.26600000 & -3.50800000 \\
\hline $\mathrm{H}$ & -5.58900000 & -7.87200000 & -3.13600000 \\
\hline C & -5.74700000 & -5.93800000 & -4.01400000 \\
\hline $\mathrm{H}$ & -6.08800000 & -5.07800000 & -3.44000000 \\
\hline $\mathrm{H}$ & -5.94600000 & -5.74300000 & -5.07400000 \\
\hline$S$ & -3.91800000 & -6.05000000 & -3.92200000 \\
\hline$S$ & -3.25900000 & -6.13000000 & -1.86000000 \\
\hline$C$ & -6.96500000 & -8.07200000 & -4.69800000 \\
\hline 0 & -8.19500000 & -8.16800000 & -4.92200000 \\
\hline $\mathrm{N}$ & -6.09300000 & -8.67100000 & -5.51300000 \\
\hline $\mathrm{H}$ & -5.08800000 & -8.64400000 & -5.31600000 \\
\hline C & -6.57800000 & -9.42200000 & -6.66600000 \\
\hline $\mathrm{H}$ & -7.17400000 & -10.27800000 & -6.33700000 \\
\hline $\mathrm{H}$ & -5.70800000 & -9.80700000 & -7.20200000 \\
\hline$C$ & -7.43200000 & -8.58900000 & -7.65500000 \\
\hline 0 & -8.28500000 & -9.25900000 & -8.32700000 \\
\hline 0 & -7.21900000 & -7.36100000 & -7.75000000 \\
\hline$F$ & -6.23400000 & -6.17900000 & -0.64000000 \\
\hline $\mathrm{O}$ & -3.42600000 & -7.28200000 & -0.43400000 \\
\hline 0 & -4.63700000 & -7.39300000 & -1.32800000 \\
\hline
\end{tabular}

\begin{tabular}{|c|c|c|c|}
\hline & -3.08800000 & -4.91000000 & 4.34100000 \\
\hline 1 & -3.66800000 & -5.60100000 & 4.95500000 \\
\hline & -3.25500000 & -3.91100000 & 4.76000000 \\
\hline & -3.31600000 & -4.05000000 & 1.94200000 \\
\hline & -2.57200000 & -3.35300000 & 1.92400000 \\
\hline & -3.58800000 & -4.98300000 & 2.93300000 \\
\hline & -4.07700000 & -4.35500000 & 0.86600000 \\
\hline & -4.07600000 & -3.77800000 & -0.04300000 \\
\hline $\mathrm{V}$ & -4.82400000 & -5.42600000 & 1.09100000 \\
\hline & -4.51900000 & -5.82600000 & 7600000 \\
\hline $\mathrm{H}$ & -4.97300000 & -6.69700000 & 2.82300000 \\
\hline & -1.53100000 & -10.44100000 & -0.46500000 \\
\hline $\mathrm{H}$ & -0.90400000 & -11.29300000 & -0.20500000 \\
\hline & -2.02900000 & -10.69100000 & -1.40800000 \\
\hline & -1.12700000 & -8.07200000 & -1.19400000 \\
\hline & -2.13900000 & -7.96900000 & -1.44000000 \\
\hline & -0.65300000 & -9.244000 & $-0.6450 c$ \\
\hline C & -0.09400000 & -7.19300000 & \\
\hline $\mathrm{H}$ & -0.19800000 & -6.18300000 & 000 \\
\hline $\mathrm{N}$ & 1.01900000 & -7.72300000 & \\
\hline C & 0.67800000 & -9.00900000 & 000 \\
\hline $\mathrm{H}$ & 1.39500000 & -9.69400000 & \\
\hline C & -9.78200000 & -3.4 & \\
\hline $\mathrm{H}$ & -10.30400000 & -2.72000000 & 2.0880 \\
\hline $\mathrm{H}$ & -8.95900000 & -2.9130 & \\
\hline $\mathrm{N}$ & -9.94500000 & -5.542 & \\
\hline $\mathrm{H}$ & -10.97200000 & -5.58600000 & \\
\hline C & -9.20300000 & -4.57600000 & \\
\hline & -9.09400000 & -6.38500000 & 0.64 \\
\hline $\mathrm{H}$ & -9.40700000 & & \\
\hline $\mathrm{N}$ & -7.83400000 & -6.01600000 & \\
\hline$c$ & -7.89800000 & & \\
\hline $\mathrm{H}$ & -7.00000000 & & \\
\hline & -7.29300000 & -2.16400 & $-0.64 C$ \\
\hline $\mathrm{H}$ & -7.0110 & & \\
\hline $\mathrm{H}$ & -8.38500000 & -2.18300000 & \\
\hline & -6.7790 & -3.2 & \\
\hline $\mathrm{O}$ & -6.90800000 & -3.01000000 & \\
\hline & -6.25 & & \\
\hline $\mathrm{N}$ & -9.19000000 & -6.26900000 & \\
\hline $\mathrm{r}$ & -8.34200000 & -5.70 & \\
\hline & -9.53700000 & -6.57900000 & -7.24800000 \\
\hline & -8.87500000 & & \\
\hline & 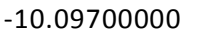 & -5.31100000 & -5.63500 \\
\hline
\end{tabular}




\begin{tabular}{|c|c|c|c|c|c|c|c|}
\hline $\mathrm{H}$ & -11.09100000 & -5.39900000 & -6.07000000 & $\mathrm{~N}$ & -4.85500000 & -5.42300000 & 1.07500000 \\
\hline C & -10.14200000 & -5.61500000 & -4.13400000 & C & -4.55800000 & -5.80700000 & 2.36700000 \\
\hline $\mathrm{H}$ & -10.65800000 & -6.56200000 & -3.95300000 & $\mathrm{H}$ & -5.03000000 & -6.65900000 & 2.83000000 \\
\hline $\mathrm{H}$ & -10.77000000 & -4.82600000 & -3.71000000 & C & -1.49200000 & -10.43600000 & -0.49600000 \\
\hline C & -8.74700000 & -5.63200000 & -3.47700000 & $\mathrm{H}$ & -0.89900000 & -11.30900000 & -0.22200000 \\
\hline $\mathrm{H}$ & -8.64500000 & -4.77500000 & -2.80400000 & $\mathrm{H}$ & -1.98300000 & -10.67000000 & -1.44700000 \\
\hline $\mathrm{H}$ & -7.96800000 & -5.44900000 & -4.21400000 & $\mathrm{~N}$ & -0.96800000 & -8.08900000 & -1.23100000 \\
\hline C & -8.42800000 & -6.85600000 & -2.60900000 & $\mathrm{H}$ & -1.95600000 & -7.91000000 & -1.50600000 \\
\hline $\mathrm{O}$ & -9.40300000 & -7.42500000 & -2.05600000 & $\mathrm{C}$ & -0.56400000 & -9.27500000 & -0.65900000 \\
\hline C & -9.55700000 & -3.87000000 & -5.90800000 & C & 0.10300000 & -7.25600000 & -1.24100000 \\
\hline $\mathrm{O}$ & -8.36400000 & -3.80600000 & -6.33000000 & $\mathrm{H}$ & 0.04900000 & -6.24400000 & -1.61700000 \\
\hline $\mathrm{O}$ & -10.36500000 & -2.95800000 & -5.65000000 & $\mathrm{~N}$ & 1.17900000 & -7.83100000 & -0.72900000 \\
\hline$N$ & -7.12500000 & -7.17700000 & -2.32000000 & C & 0.77000000 & -9.09600000 & -0.36300000 \\
\hline $\mathrm{C}$ & -6.19100000 & -7.29100000 & -3.45400000 & $\mathrm{H}$ & 1.44200000 & -9.80800000 & 0.09400000 \\
\hline $\mathrm{H}$ & -5.34300000 & -7.86600000 & -3.06700000 & C & -9.80200000 & -3.43500000 & 2.73600000 \\
\hline C & -5.59400000 & -5.96500000 & -4.05100000 & $\mathrm{H}$ & -10.32700000 & -2.70400000 & 2.10700000 \\
\hline $\mathrm{H}$ & -5.95600000 & -5.08000000 & -3.52500000 & $\mathrm{H}$ & -8.97600000 & -2.90200000 & 3.21700000 \\
\hline $\mathrm{H}$ & -5.81500000 & -5.84200000 & -5.11700000 & $\mathrm{~N}$ & -9.98100000 & -5.52300000 & 1.27000000 \\
\hline$S$ & -3.75600000 & -5.94900000 & -3.98700000 & $\mathrm{H}$ & -11.00800000 & -5.56200000 & 1.22300000 \\
\hline$S$ & -3.33600000 & -5.59200000 & -2.00900000 & C & -9.23100000 & -4.56300000 & 1.93000000 \\
\hline C & -6.79200000 & -8.12000000 & -4.60600000 & $\mathrm{C}$ & -9.13800000 & -6.37000000 & 0.64900000 \\
\hline $\mathrm{O}$ & -8.03200000 & -8.21300000 & -4.77500000 & $\mathrm{H}$ & -9.45800000 & -7.18500000 & 0.02000000 \\
\hline $\mathrm{N}$ & -5.96000000 & -8.71200000 & -5.46500000 & $\mathrm{~N}$ & -7.87500000 & -6.01100000 & 0.85100000 \\
\hline $\mathrm{H}$ & -4.94500000 & -8.67000000 & -5.33600000 & $\mathrm{C}$ & -7.92900000 & -4.89400000 & 1.66800000 \\
\hline C & -6.49300000 & -9.43700000 & -6.61200000 & $\mathrm{H}$ & -7.02700000 & -4.40100000 & 1.98900000 \\
\hline $\mathrm{H}$ & -7.10700000 & -10.27800000 & -6.27700000 & C & -7.30300000 & -2.15700000 & -0.63400000 \\
\hline $\mathrm{H}$ & -5.64700000 & -9.84200000 & -7.17200000 & $\mathrm{H}$ & -7.00900000 & -2.42200000 & 0.38200000 \\
\hline C & -7.33900000 & -8.56300000 & -7.57100000 & $\mathrm{H}$ & -8.39600000 & -2.17300000 & -0.69100000 \\
\hline $\mathrm{O}$ & -8.21900000 & -9.19300000 & -8.24300000 & C & -6.80600000 & -3.19600000 & -1.65100000 \\
\hline $\mathrm{O}$ & -7.09400000 & -7.33900000 & -7.63800000 & 0 & -6.94500000 & -2.99300000 & -2.86200000 \\
\hline $\mathrm{Fe}$ & -6.21400000 & -6.17100000 & -0.61200000 & $\mathrm{O}$ & -6.32300000 & -4.26800000 & -1.12600000 \\
\hline $\mathrm{O}$ & -3.79000000 & -8.19100000 & -1.63900000 & $\mathrm{~N}$ & -9.18900000 & -6.26400000 & -6.33700000 \\
\hline \multirow[t]{2}{*}{$\mathrm{O}$} & -4.18600000 & -6.87700000 & -1.21700000 & $\mathrm{H}$ & -8.33900000 & -5.70400000 & -6.53500000 \\
\hline & & & & $\mathrm{H}$ & -9.52600000 & -6.57200000 & -7.25400000 \\
\hline \multicolumn{2}{|c|}{${ }^{5} \mathrm{TS3} b$} & & & $\mathrm{H}$ & -8.88000000 & -7.09100000 & -5.78300000 \\
\hline \multicolumn{2}{|c|}{-4312.054909 (a.u.) } & & & $\mathrm{C}$ & -10.10400000 & -5.30900000 & -5.64400000 \\
\hline C & -3.10500000 & -4.89800000 & 4.31700000 & $\mathrm{H}$ & -11.09300000 & -5.39600000 & -6.08800000 \\
\hline $\mathrm{H}$ & -3.68800000 & -5.58400000 & 4.93300000 & C & -10.16400000 & -5.61600000 & -4.14400000 \\
\hline $\mathrm{H}$ & -3.27000000 & -3.89600000 & 4.73100000 & $\mathrm{H}$ & -10.68200000 & -6.56300000 & -3.97100000 \\
\hline $\mathrm{N}$ & -3.31100000 & -4.06900000 & 1.89900000 & $\mathrm{H}$ & -10.79700000 & -4.82800000 & -3.72500000 \\
\hline $\mathrm{H}$ & -2.55600000 & -3.38400000 & 1.87200000 & C & -8.77600000 & -5.63500000 & -3.47100000 \\
\hline C & -3.60500000 & -4.97800000 & 2.90900000 & $\mathrm{H}$ & -8.68200000 & -4.77700000 & -2.79700000 \\
\hline C & -4.08000000 & -4.37500000 & 0.82900000 & $\mathrm{H}$ & -7.98700000 & -5.45300000 & -4.19700000 \\
\hline $\mathrm{H}$ & -4.05000000 & -3.83300000 & -0.10100000 & $\mathrm{C}$ & -8.47000000 & -6.85700000 & -2.59700000 \\
\hline
\end{tabular}




\begin{tabular}{|c|c|c|c|c|c|c|c|}
\hline 0 & -9.44900000 & -7.42200000 & -2.05000000 & C & -0.37100000 & -9.32000000 & -0.48300000 \\
\hline C & -9.56300000 & -3.86600000 & -5.90900000 & C & 0.14200000 & -7.43100000 & -1.53500000 \\
\hline 0 & -8.36900000 & -3.80000000 & -6.32800000 & $\mathrm{H}$ & 0.04000000 & -6.58300000 & -2.19900000 \\
\hline $\mathrm{O}$ & -10.37300000 & -2.95500000 & -5.65000000 & $\mathrm{~N}$ & 1.14700000 & -7.65400000 & -0.71400000 \\
\hline $\mathrm{N}$ & -7.16900000 & -7.18400000 & -2.29700000 & C & 0.83900000 & -8.82900000 & -0.06200000 \\
\hline C & -6.22500000 & -7.29500000 & -3.42400000 & $\mathrm{H}$ & 1.49800000 & -9.26500000 & 0.67400000 \\
\hline $\mathrm{H}$ & -5.38100000 & -7.87500000 & -3.04100000 & C & -9.78800000 & -3.44600000 & 2.72000000 \\
\hline C & -5.61300000 & -5.96600000 & -3.99100000 & $\mathrm{H}$ & -10.30100000 & -2.70400000 & 2.09400000 \\
\hline $\mathrm{H}$ & -5.95400000 & -5.09100000 & -3.43500000 & $\mathrm{H}$ & -8.95300000 & -2.92800000 & 3.20200000 \\
\hline $\mathrm{H}$ & -5.84400000 & -5.80500000 & -5.05100000 & $\mathrm{~N}$ & -10.02300000 & -5.50700000 & 1.23300000 \\
\hline$S$ & -3.78200000 & -5.99900000 & -3.95700000 & $\mathrm{H}$ & -11.05000000 & -5.52200000 & 1.19700000 \\
\hline$S$ & -3.33200000 & -5.89500000 & -1.92200000 & $\mathrm{C}$ & -9.24200000 & -4.58200000 & 1.90800000 \\
\hline C & -6.80800000 & -8.11400000 & -4.59000000 & C & -9.20900000 & -6.37600000 & 0.60400000 \\
\hline $\mathrm{O}$ & -8.04600000 & -8.21600000 & -4.76900000 & $\mathrm{H}$ & -9.55600000 & -7.16800000 & -0.03900000 \\
\hline $\mathrm{N}$ & -5.96400000 & -8.69300000 & -5.44800000 & $\mathrm{~N}$ & -7.93700000 & -6.06400000 & 0.81400000 \\
\hline $\mathrm{H}$ & -4.95000000 & -8.63500000 & -5.32100000 & C & -7.95100000 & -4.95600000 & 1.64200000 \\
\hline C & -6.48600000 & -9.42000000 & -6.60000000 & $\mathrm{H}$ & -7.03100000 & -4.49700000 & 1.96800000 \\
\hline $\mathrm{H}$ & -7.09200000 & -10.26800000 & -6.26900000 & C & -7.24700000 & -2.24200000 & -0.57900000 \\
\hline $\mathrm{H}$ & -5.63100000 & -9.81500000 & -7.15500000 & $\mathrm{H}$ & -6.88600000 & -2.50700000 & 0.41500000 \\
\hline C & -7.33700000 & -8.55700000 & -7.56400000 & $\mathrm{H}$ & -8.34200000 & -2.25100000 & -0.55500000 \\
\hline 0 & -8.21400000 & -9.19500000 & -8.23500000 & $\mathrm{C}$ & -6.84000000 & -3.30200000 & -1.62100000 \\
\hline $\mathrm{O}$ & -7.10000000 & -7.33100000 & -7.63600000 & $\mathrm{O}$ & -7.02300000 & -3.07700000 & -2.82600000 \\
\hline $\mathrm{Fe}$ & -6.28300000 & -6.14800000 & -0.60300000 & 0 & -6.40100000 & -4.39500000 & -1.11800000 \\
\hline 0 & -3.58100000 & -7.98900000 & -1.90700000 & $\mathrm{~N}$ & -9.21000000 & -6.22100000 & -6.53300000 \\
\hline \multirow[t]{2}{*}{0} & -4.21600000 & -6.98400000 & -1.09800000 & $\mathrm{H}$ & -8.31900000 & -5.70800000 & -6.66600000 \\
\hline & & & & $\mathrm{H}$ & -9.52200000 & -6.45900000 & -7.48100000 \\
\hline \multicolumn{2}{|c|}{${ }^{5} \mathrm{IM} 3 \mathrm{~b}$} & & & $\mathrm{H}$ & -8.98600000 & -7.09100000 & -6.01100000 \\
\hline \multicolumn{2}{|c|}{-4312.219797 (a.u.) } & & & $\mathrm{C}$ & -10.09400000 & -5.24000000 & -5.83400000 \\
\hline C & -3.13700000 & -4.86700000 & 4.16500000 & $\mathrm{H}$ & -11.08200000 & -5.28700000 & -6.28600000 \\
\hline $\mathrm{H}$ & -3.75700000 & -5.52400000 & 4.77900000 & $\mathrm{C}$ & -10.16100000 & -5.57300000 & -4.34400000 \\
\hline $\mathrm{H}$ & -3.34400000 & -3.84300000 & 4.50200000 & $\mathrm{H}$ & -10.71500000 & -6.50200000 & -4.19200000 \\
\hline $\mathrm{N}$ & -3.13200000 & -4.26200000 & 1.65700000 & $\mathrm{H}$ & -10.75700000 & -4.77500000 & -3.89100000 \\
\hline $\mathrm{H}$ & -2.38100000 & -3.56900000 & 1.62800000 & C & -8.76800000 & -5.67300000 & -3.69100000 \\
\hline C & -3.53800000 & -5.04600000 & 2.73200000 & $\mathrm{H}$ & -8.55900000 & -4.77500000 & -3.10000000 \\
\hline C & -3.85700000 & -4.63800000 & 0.57600000 & $\mathrm{H}$ & -7.97000000 & -5.65700000 & -4.43600000 \\
\hline $\mathrm{H}$ & -3.75200000 & -4.18400000 & -0.39700000 & C & -8.59900000 & -6.85600000 & -2.74200000 \\
\hline $\mathrm{N}$ & -4.70600000 & -5.61000000 & 0.87600000 & $\mathrm{O}$ & -9.64200000 & -7.27000000 & -2.16700000 \\
\hline C & -4.50300000 & -5.87600000 & 2.21400000 & C & -9.50100000 & -3.81700000 & -6.06300000 \\
\hline $\mathrm{H}$ & -5.04500000 & -6.65700000 & 2.72300000 & $\mathrm{O}$ & -8.30000000 & -3.78200000 & -6.46500000 \\
\hline C & -1.13300000 & -10.56100000 & -0.15700000 & 0 & -10.28400000 & -2.88700000 & -5.79000000 \\
\hline $\mathrm{H}$ & -0.42900000 & -11.31100000 & 0.19200000 & $\mathrm{~N}$ & -7.37000000 & -7.33300000 & -2.40900000 \\
\hline $\mathrm{H}$ & -1.58100000 & -10.94600000 & -1.07400000 & C & -6.39500000 & -7.65500000 & -3.44500000 \\
\hline $\mathrm{N}$ & -0.79600000 & -8.41700000 & -1.44100000 & $\mathrm{H}$ & -5.80700000 & -8.49100000 & -3.04400000 \\
\hline $\mathrm{H}$ & -1.68100000 & -8.49700000 & -1.92400000 & C & -5.36400000 & -6.55300000 & -3.81100000 \\
\hline
\end{tabular}




\begin{tabular}{|c|c|c|c|c|c|c|c|}
\hline $\mathrm{H}$ & -5.85700000 & -5.58400000 & -3.91500000 & $\mathrm{H}$ & -9.05100000 & -3.12500000 & 3.13300000 \\
\hline $\mathrm{H}$ & -4.85700000 & -6.79700000 & -4.74400000 & $\mathrm{~N}$ & -9.91900000 & -5.89900000 & 1.32900000 \\
\hline$S$ & -4.07000000 & -6.30200000 & -2.52200000 & $\mathrm{H}$ & -10.94000000 & -5.96400000 & 1.25900000 \\
\hline S & -4.05200000 & -8.09100000 & -1.01600000 & C & -9.23500000 & -4.85700000 & 1.94200000 \\
\hline C & -7.00200000 & -8.26900000 & -4.73300000 & C & -9.02300000 & -6.66600000 & 0.67900000 \\
\hline $\mathrm{O}$ & -8.23400000 & -8.29500000 & -4.94700000 & $\mathrm{H}$ & -9.29300000 & -7.51900000 & 0.07700000 \\
\hline $\mathrm{N}$ & -6.16700000 & -8.83600000 & -5.61200000 & $\mathrm{~N}$ & -7.79100000 & -6.20200000 & 0.85300000 \\
\hline $\mathrm{H}$ & -5.15500000 & -8.81400000 & -5.47900000 & C & -7.91900000 & -5.07800000 & 1.65200000 \\
\hline C & -6.70300000 & -9.55700000 & -6.75800000 & $\mathrm{H}$ & -7.05700000 & -4.49200000 & 1.92500000 \\
\hline $\mathrm{H}$ & -7.35000000 & -10.36900000 & -6.41400000 & C & -7.34700000 & -1.98200000 & -0.61100000 \\
\hline $\mathrm{H}$ & -5.86000000 & -10.00600000 & -7.28900000 & $\mathrm{H}$ & -7.16300000 & -2.17600000 & 0.44700000 \\
\hline C & -7.50100000 & -8.68800000 & -7.75500000 & $\mathrm{H}$ & -8.42800000 & -2.00400000 & -0.76900000 \\
\hline 0 & -8.36400000 & -9.32600000 & -8.44300000 & C & -6.76200000 & -3.12500000 & -1.45500000 \\
\hline $\mathrm{O}$ & -7.23900000 & -7.46900000 & -7.84400000 & $\mathrm{O}$ & -6.60100000 & -3.02200000 & -2.67800000 \\
\hline $\mathrm{Fe}$ & -6.37900000 & -6.31100000 & -0.65800000 & $\mathrm{O}$ & -6.56300000 & -4.16900000 & -0.73700000 \\
\hline $\mathrm{O}$ & -3.68700000 & -9.32900000 & -1.81900000 & $\mathrm{~N}$ & -9.33800000 & -5.85700000 & -6.72000000 \\
\hline \multirow[t]{2}{*}{0} & -5.48900000 & -8.22100000 & -0.50500000 & $\mathrm{H}$ & -8.44400000 & -5.35800000 & -6.85800000 \\
\hline & & & & $\mathrm{H}$ & -9.66200000 & -6.10000000 & -7.66400000 \\
\hline \multicolumn{2}{|c|}{${ }^{5} \mathrm{TS} 1 \mathrm{c}$} & & & $\mathrm{H}$ & -9.12800000 & -6.72300000 & -6.18100000 \\
\hline \multicolumn{2}{|c|}{-4312.076944 (a.u.) } & & & C & -10.19400000 & -4.86900000 & -6.00500000 \\
\hline$C$ & -3.10000000 & -4.85500000 & 4.16300000 & $\mathrm{H}$ & -11.19100000 & -4.91100000 & -6.43500000 \\
\hline $\mathrm{H}$ & -3.71500000 & -5.53200000 & 4.76000000 & C & -10.19100000 & -5.19500000 & -4.51300000 \\
\hline $\mathrm{H}$ & -3.29300000 & -3.84500000 & 4.54600000 & $\mathrm{H}$ & -10.54100000 & -6.22000000 & -4.35600000 \\
\hline $\mathrm{N}$ & -3.13000000 & -4.13600000 & 1.69000000 & $\mathrm{H}$ & -10.90900000 & -4.53200000 & -4.02800000 \\
\hline $\mathrm{H}$ & -2.36400000 & -3.45800000 & 1.67600000 & C & -8.77900000 & -5.00400000 & -3.93100000 \\
\hline C & -3.52500000 & -4.97000000 & 2.73000000 & $\mathrm{H}$ & -8.61800000 & -3.95300000 & -3.67200000 \\
\hline C & -3.87300000 & -4.44800000 & 0.60200000 & $\mathrm{H}$ & -8.01400000 & -5.23200000 & -4.66900000 \\
\hline $\mathrm{H}$ & -3.78200000 & -3.94100000 & -0.34600000 & C & -8.59000000 & -5.79100000 & -2.64900000 \\
\hline $\mathrm{N}$ & -4.71900000 & -5.43300000 & 0.86700000 & $\mathrm{O}$ & -9.46700000 & -5.63300000 & -1.78100000 \\
\hline C & -4.50300000 & -5.77000000 & & C & & & -6.21500000 \\
\hline $\mathrm{H}$ & -5.05200000 & -6.56700000 & 2.66600000 & 0 & -8.38800000 & -3.41600000 & -6.61800000 \\
\hline C & -1.78000000 & -10.10700000 & -0.43600000 & 0 & & & -5.88500000 \\
\hline $\mathrm{H}$ & -1.07700000 & -10.94200000 & -0.40100000 & $\mathrm{~N}$ & -7.49300000 & -6.59000000 & -2.44500000 \\
\hline $\mathrm{H}$ & -2.39100000 & -10.23800000 & -1.33800000 & C & -6.63600000 & -7.01900000 & -3.55900000 \\
\hline $\mathrm{N}$ & -1.47700000 & -7.56800000 & -0.57100000 & $\mathrm{H}$ & -6.06500000 & -7.84600000 & -3.12000000 \\
\hline $\mathrm{H}$ & -2.46900000 & -7.34300000 & -0.53700000 & C & -5.54000000 & -6.01300000 & -3.97800000 \\
\hline C & -0.96400000 & -8.85200000 & -0.51400000 & $\mathrm{H}$ & -5.89700000 & -4.98700000 & -4.12100000 \\
\hline C & -0.42800000 & -6.70700000 & -0.66600000 & $\mathrm{H}$ & -5.03500000 & -6.35000000 & -4.88700000 \\
\hline $\mathrm{H}$ & -0.55000000 & -5.63300000 & -0.71200000 & $\mathrm{~S}$ & -4.28800000 & -5.92900000 & -2.59100000 \\
\hline $\mathrm{N}$ & 0.72500000 & -7.35000000 & -0.68800000 & $S$ & -4.71100000 & -7.72900000 & -1.33300000 \\
\hline C & 0.40000000 & -8.68500000 & & C & -7.29000000 & -7.74400000 & -4.74000000 \\
\hline $\mathrm{H}$ & 1.15300000 & -9.46200000 & -0.58400000 & 0 & -8.52300000 & -7.73100000 & -4.95700000 \\
\hline C & -9.85900000 & -3.72000000 & 2.69400000 & $\mathrm{~N}$ & -6.47100000 & -8.49500000 & -5.49200000 \\
\hline $\mathrm{H}$ & -10.41200000 & -3.04600000 & 2.02600000 & $\mathrm{H}$ & -5.47400000 & -8.54500000 & -5.27400000 \\
\hline
\end{tabular}




\begin{tabular}{|c|c|c|c|c|c|c|c|}
\hline C & -7.00600000 & -9.32100000 & -6.56600000 & $\mathrm{H}$ & -7.07800000 & -4.19000000 & 1.90000000 \\
\hline $\mathrm{H}$ & -7.74100000 & -10.02700000 & -6.16600000 & C & -7.12200000 & -2.21500000 & -0.66100000 \\
\hline $\mathrm{H}$ & -6.18200000 & -9.90600000 & -6.98500000 & $\mathrm{H}$ & -6.79500000 & -2.50600000 & 0.33700000 \\
\hline C & -7.68100000 & -8.53400000 & -7.71100000 & $\mathrm{H}$ & -8.21000000 & -2.29500000 & -0.70700000 \\
\hline 0 & -8.47300000 & -9.23000000 & -8.42700000 & C & -6.55500000 & -3.16100000 & -1.73900000 \\
\hline $\mathrm{O}$ & -7.40400000 & -7.32600000 & -7.87000000 & $\mathrm{O}$ & -6.77100000 & -2.90000000 & -2.93400000 \\
\hline $\mathrm{Fe}$ & -6.31200000 & -6.13000000 & -0.69600000 & $\mathrm{O}$ & -5.91000000 & -4.18200000 & -1.30900000 \\
\hline $\mathrm{O}$ & -6.72500000 & -10.41900000 & -0.36300000 & $\mathrm{~N}$ & -9.39800000 & -6.22500000 & -6.45900000 \\
\hline \multirow[t]{2}{*}{$\mathrm{O}$} & -5.99700000 & -9.61700000 & 0.33500000 & $\mathrm{H}$ & -8.49900000 & -5.74600000 & -6.64200000 \\
\hline & & & & $\mathrm{H}$ & -9.75000000 & -6.50700000 & -7.37900000 \\
\hline \multicolumn{2}{|c|}{${ }^{5}$ IM1C } & & & $\mathrm{H}$ & -9.20300000 & -7.06100000 & -5.88000000 \\
\hline \multicolumn{2}{|c|}{4312.099299 (a.u.) } & & & C & -10.25200000 & -5.20200000 & -5.77400000 \\
\hline C & -3.16000000 & -4.93400000 & 4.20400000 & $\mathrm{H}$ & -11.26500000 & -5.29200000 & -6.15600000 \\
\hline $\mathrm{H}$ & -3.77800000 & -5.61100000 & 4.79600000 & $\mathrm{C}$ & -10.19200000 & -5.44800000 & -4.26700000 \\
\hline $\mathrm{H}$ & -3.32600000 & -3.92900000 & 4.61100000 & $\mathrm{H}$ & -10.55100000 & -6.45900000 & -4.04500000 \\
\hline $\mathrm{N}$ & -3.22200000 & -4.14200000 & 1.75700000 & $\mathrm{H}$ & -10.88100000 & -4.74600000 & -3.79700000 \\
\hline $\mathrm{H}$ & -2.43700000 & -3.48700000 & 1.74300000 & C & -8.76100000 & -5.25800000 & -3.74300000 \\
\hline C & -3.61700000 & -4.99800000 & 2.77800000 & $\mathrm{H}$ & -8.55200000 & -4.19200000 & -3.60400000 \\
\hline C & -4.00900000 & -4.37100000 & 0.68400000 & $\mathrm{H}$ & -8.03400000 & -5.58700000 & -4.48000000 \\
\hline $\mathrm{H}$ & -3.93500000 & -3.83800000 & -0.24900000 & C & -8.54600000 & -5.89800000 & -2.37900000 \\
\hline $\mathrm{N}$ & -4.89500000 & -5.32900000 & 0.94200000 & $\mathrm{O}$ & -9.42200000 & -5.63100000 & -1.53400000 \\
\hline C & -4.64500000 & -5.73500000 & 2.23800000 & C & -9.67800000 & -3.79200000 & -6.10400000 \\
\hline $\mathrm{H}$ & -5.21000000 & -6.52900000 & 2.69700000 & 0 & -8.48200000 & -3.76800000 & -6.51800000 \\
\hline C & -1.60900000 & -10.22400000 & -0.36000000 & 0 & -10.46200000 & -2.85600000 & -5.85900000 \\
\hline $\mathrm{H}$ & -1.00200000 & -11.12800000 & -0.29400000 & $\mathrm{~N}$ & -7.42600000 & -6.63800000 & -2.09400000 \\
\hline $\mathrm{H}$ & -2.14600000 & -10.25500000 & -1.31200000 & C & -6.59300000 & -7.14800000 & -3.19600000 \\
\hline $\mathrm{N}$ & -1.01200000 & -7.72400000 & -0.38300000 & $\mathrm{H}$ & -5.97100000 & -7.93200000 & -2.73100000 \\
\hline $\mathrm{H}$ & -1.94700000 & -7.35600000 & -0.52400000 & C & -5.67300000 & -6.07700000 & -3.84700000 \\
\hline C & -0.66800000 & -9.06200000 & -0.27000000 & $\mathrm{H}$ & -6.05100000 & -5.07600000 & -3.64600000 \\
\hline C & 0.11700000 & -6.98500000 & -0.22500000 & $\mathrm{H}$ & -5.57900000 & -6.22500000 & -4.92600000 \\
\hline $\mathrm{H}$ & 0.12500000 & -5.90300000 & -0.26100000 & $S$ & -3.93900000 & -6.08300000 & -3.20400000 \\
\hline $\mathrm{N}$ & 1.17300000 & -7.75500000 & -0.03300000 & $S$ & -4.15700000 & -6.96800000 & -1.31800000 \\
\hline C & 0.69200000 & -9.04800000 & -0.06500000 & C & -7.27800000 & -7.99000000 & -4.29400000 \\
\hline $\mathrm{H}$ & 1.33700000 & -9.90600000 & 0.06000000 & 0 & -8.51600000 & -8.08600000 & -4.44600000 \\
\hline C & -9.89700000 & -3.55600000 & 2.68800000 & $\mathrm{~N}$ & -6.41900000 & -8.65000000 & -5.09400000 \\
\hline $\mathrm{H}$ & -10.48100000 & -2.91900000 & 2.01100000 & $\mathrm{H}$ & -5.41700000 & -8.59500000 & -4.93000000 \\
\hline $\mathrm{H}$ & -9.11300000 & -2.92100000 & 3.11400000 & C & -6.87500000 & -9.44100000 & -6.22400000 \\
\hline $\mathrm{N}$ & -9.84600000 & -5.81800000 & 1.45100000 & $\mathrm{H}$ & -7.56000000 & -10.22700000 & -5.89300000 \\
\hline $\mathrm{H}$ & -10.85900000 & -5.96300000 & 1.39300000 & $\mathrm{H}$ & -6.00100000 & -9.93300000 & -6.66000000 \\
\hline C & -9.22700000 & -4.68500000 & 1.96400000 & C & -7.55900000 & -8.60000000 & -7.33000000 \\
\hline C & -8.91300000 & -6.56600000 & 0.83600000 & $\mathrm{O}$ & -8.50100000 & -9.20000000 & -7.96900000 \\
\hline $\mathrm{H}$ & -9.11900000 & -7.49200000 & 0.32500000 & $\mathrm{O}$ & -7.17300000 & -7.43200000 & -7.52300000 \\
\hline$N$ & -7.71100000 & -6.00800000 & 0.94400000 & $\mathrm{Fe}$ & -6.25600000 & -6.01500000 & -0.50600000 \\
\hline C & -7.90400000 & -4.83800000 & 1.66000000 & 0 & -5.20600000 & -9.39300000 & -1.73200000 \\
\hline
\end{tabular}




\begin{tabular}{|c|c|c|c|c|c|c|c|}
\hline \multirow[t]{2}{*}{$\mathrm{O}$} & \multirow[t]{2}{*}{-4.01300000} & \multirow[t]{2}{*}{-8.63000000} & \multirow[t]{2}{*}{-1.45600000} & $\mathrm{H}$ & -8.26700000 & -5.75800000 & -6.76500000 \\
\hline & & & & $\mathrm{H}$ & -9.50700000 & -6.54100000 & -7.52000000 \\
\hline${ }^{5} \mathrm{TS}_{2}$ & & & & $\mathrm{H}$ & -8.98400000 & -7.08600000 & -6.00800000 \\
\hline-431 & 12.084283 (a.u.) & & & C. & -10.02600000 & -5.22600000 & -5.93000000 \\
\hline C & -2.99500000 & -4.93200000 & 4.36500000 & $\mathrm{H}$ & -11.03800000 & -5.33000000 & -6.31300000 \\
\hline $\mathrm{H}$ & -3.61300000 & -5.60900000 & 4.95800000 & $\mathrm{C}$ & -9.96000000 & -5.45000000 & -4.42400000 \\
\hline $\mathrm{H}$ & -3.15700000 & -3.92800000 & 4.77400000 & $\mathrm{H}$ & -10.33700000 & -6.44900000 & -4.18300000 \\
\hline $\mathrm{N}$ & -3.03500000 & -4.18000000 & 1.89800000 & $\mathrm{H}$ & -10.63200000 & -4.72700000 & -3.95600000 \\
\hline $\mathrm{H}$ & -2.26400000 & -3.50800000 & 1.87400000 & C & -8.52900000 & -5.27000000 & -3.88800000 \\
\hline C & -3.44700000 & -5.00600000 & 2.94000000 & $\mathrm{H}$ & -8.31600000 & -4.20500000 & -3.75600000 \\
\hline C & -3.78300000 & -4.47400000 & 0.81200000 & $\mathrm{H}$ & -7.77400000 & -5.63500000 & -4.58300000 \\
\hline $\mathrm{H}$ & -3.69300000 & -3.97000000 & -0.13700000 & $\mathrm{C}$ & -8.39100000 & -5.88100000 & -2.50700000 \\
\hline $\mathrm{N}$ & -4.65800000 & -5.43500000 & 1.08100000 & 0 & -9.27400000 & -5.53400000 & -1.69400000 \\
\hline $\mathrm{C}$ & -4.44700000 & -5.77600000 & 2.40000000 & $\mathrm{C}$ & -9.45300000 & -3.82100000 & -6.27300000 \\
\hline $\mathrm{H}$ & -5.01400000 & -6.55900000 & 2.88000000 & 0 & -8.25100000 & -3.80700000 & -6.68000000 \\
\hline C & -1.57100000 & -10.22200000 & -0.26100000 & $\mathrm{O}$ & -10.23100000 & -2.87900000 & -6.04000000 \\
\hline $\mathrm{H}$ & -0.91200000 & -11.09100000 & -0.32300000 & $\mathrm{~N}$ & -7.34600000 & -6.67700000 & -2.14200000 \\
\hline $\mathrm{H}$ & -2.17300000 & -10.20000000 & -1.17700000 & $\mathrm{C}$ & -6.48800000 & -7.42300000 & -3.06600000 \\
\hline $\mathrm{N}$ & -1.14300000 & -7.70600000 & -0.01200000 & $\mathrm{H}$ & -6.23500000 & -8.34200000 & -2.52400000 \\
\hline $\mathrm{H}$ & -2.11700000 & -7.43500000 & 0.06300000 & $\mathrm{C}$ & -5.11000000 & -6.80600000 & -3.41800000 \\
\hline $\mathrm{C}$ & -0.69700000 & -9.01200000 & -0.13500000 & $\mathrm{H}$ & -5.20800000 & -5.87300000 & -3.98300000 \\
\hline C & -0.05400000 & -6.89700000 & 0.07700000 & $\mathrm{H}$ & -4.57300000 & -7.54100000 & -4.00300000 \\
\hline $\mathrm{H}$ & -0.12300000 & -5.82200000 & 0.18400000 & S & -3.87400000 & -6.45400000 & -2.08200000 \\
\hline $\mathrm{N}$ & 1.06500000 & -7.59300000 & 0.00200000 & $S$ & -4.58800000 & -7.86400000 & -0.61100000 \\
\hline C & 0.67400000 & -8.90800000 & -0.13300000 & $\mathrm{C}$ & -7.13300000 & -8.05900000 & -4.33000000 \\
\hline $\mathrm{H}$ & 1.38600000 & -9.71700000 & -0.21400000 & $\mathrm{O}$ & -8.37000000 & -8.08400000 & -4.49600000 \\
\hline C & -9.86300000 & -3.47400000 & 2.75500000 & $\mathrm{~N}$ & -6.32300000 & -8.71100000 & -5.19400000 \\
\hline $\mathrm{H}$ & -10.42500000 & -2.79500000 & 2.10000000 & $\mathrm{H}$ & -5.31700000 & -8.71800000 & -5.07700000 \\
\hline $\mathrm{H}$ & -9.06600000 & -2.87900000 & 3.21300000 & C & -6.86800000 & -9.53400000 & -6.26400000 \\
\hline $\mathrm{N}$ & -9.89400000 & -5.66100000 & 1.40700000 & $\mathrm{H}$ & -7.60600000 & -10.23500000 & -5.86500000 \\
\hline $\mathrm{H}$ & -10.91200000 & -5.76600000 & 1.32800000 & $\mathrm{H}$ & -6.04500000 & -10.12600000 & -6.67300000 \\
\hline C & -9.22500000 & -4.59900000 & 1.99900000 & C & -7.50100000 & -8.72800000 & -7.42500000 \\
\hline C & -8.98500000 & -6.46300000 & 0.82500000 & $\mathrm{O}$ & -8.54700000 & -9.24900000 & -7.95300000 \\
\hline $\mathrm{H}$ & -9.23800000 & -7.34900000 & 0.26600000 & 0 & -6.95900000 & -7.66000000 & -7.77900000 \\
\hline $\mathrm{N}$ & -7.75500000 & -6.00000000 & 1.01600000 & $\mathrm{Fe}$ & -6.24500000 & -5.94000000 & -0.49200000 \\
\hline C & -7.90100000 & -4.84200000 & 1.75900000 & 0 & -5.52400000 & -9.76000000 & -0.52700000 \\
\hline $\mathrm{H}$ & -7.04100000 & -4.26300000 & 2.05600000 & $\mathrm{O}$ & -4.61500000 & -9.21600000 & -1.59500000 \\
\hline $\mathrm{C}$ & -7.18000000 & -2.12100000 & -0.57400000 & & & & \\
\hline $\mathrm{H}$ & -6.89200000 & -2.38300000 & 0.44500000 & ${ }^{5} \mathrm{IM} 2$ & & & \\
\hline $\mathrm{H}$ & -8.26600000 & -2.19100000 & -0.66200000 & -431 & 12.136138 (a.u.) & & \\
\hline C & -6.57700000 & -3.09900000 & -1.59300000 & C & -2.98000000 & -5.01200000 & 4.47400000 \\
\hline 0 & -6.74500000 & -2.90200000 & -2.80100000 & $\mathrm{H}$ & -3.57400000 & -5.70800000 & 5.06800000 \\
\hline $\mathrm{O}$ & -5.93300000 & -4.08700000 & -1.07500000 & $\mathrm{H}$ & -3.13800000 & -4.02100000 & 4.91500000 \\
\hline $\mathrm{N}$ & -9.16100000 & -6.25600000 & -6.59800000 & $\mathrm{~N}$ & -3.19700000 & -4.08700000 & 2.09300000 \\
\hline
\end{tabular}




\begin{tabular}{|c|c|c|c|c|c|c|c|}
\hline $\mathrm{H}$ & -2.44300000 & -3.39900000 & 2.07600000 & C & -8.85000000 & -5.28000000 & -3.73300000 \\
\hline C & -3.47100000 & -5.04700000 & 3.06100000 & $\mathrm{H}$ & -8.68400000 & -4.23200000 & -3.46300000 \\
\hline & -3.95700000 & -4.37000000 & 1.00700000 & $\mathrm{H}$ & -8.09200000 & -5.49000000 & -4.48200000 \\
\hline & -3.96000000 & -3.76100000 & 0.11800000 & C & -8.65000000 & -6.09500000 & -2.45900000 \\
\hline & -4.70400000 & -5.44200000 & 1.20500000 & 0 & -9.57900000 & -5.99200000 & -1.62700000 \\
\hline & -4.40300000 & -5.87200000 & 2.47900000 & C & -9.63100000 & -3.74300000 & -6.09400000 \\
\hline & -4.87300000 & -6.74500000 & 2.90500000 & 0 & -8.42500000 & -3.72000000 & -6.48800000 \\
\hline & -1.78600000 & -10.07600000 & -0.38000000 & 0 & -10.40500000 & -2.80600000 & -5.82800000 \\
\hline & -1.23800000 & -11.01400000 & -0.48300000 & $\mathrm{~N}$ & -7.51300000 & -6.80300000 & -2.22600000 \\
\hline & -2.38800000 & -9.94500000 & -1.28600000 & C & -6.60800000 & -7.13000000 & -3.32600000 \\
\hline & -1.04400000 & -7.64000000 & -0.09900000 & $\mathrm{H}$ & -5.84800000 & -7.75900000 & -2.86300000 \\
\hline & -1.98200000 & -7.25100000 & -0.14500000 & C & -5.82400000 & -5.93100000 & -3.95600000 \\
\hline & -0.76400000 & -8.99100000 & -0.20700000 & $\mathrm{H}$ & -6.10900000 & -4.99000000 & -3.48000000 \\
\hline & 0.12800000 & -6.98000000 & 0.07200000 & $\mathrm{H}$ & -5.98100000 & -5.83200000 & -5.03600000 \\
\hline & 0.19200000 & -5.90400000 & 0.17300000 & $S$ & -3.98600000 & -6.05600000 & -3.84200000 \\
\hline & 1.15400000 & -7.81500000 & 0.07600000 & $S$ & -3.53700000 & -5.75800000 & -1.86400000 \\
\hline & 0.60700000 & -9.06900000 & -0.10000000 & C & -7.22000000 & -8.00100000 & -4.44300000 \\
\hline & 1.21400000 & -9.96300000 & -0.13800000 & 0 & -8.45200000 & -8.08000000 & -4.64700000 \\
\hline & -9.87400000 & -3.63100000 & 2.71600000 & $\mathrm{~N}$ & -6.34900000 & -8.66400000 & -5.22500000 \\
\hline - & -10.43900000 & -2.98300000 & 2.03200000 & $\mathrm{H}$ & -5.34800000 & -8.64800000 & -5.02100000 \\
\hline $\mathrm{H}$ & -9.07200000 & -3.01800000 & 3.13900000 & C & -6.80300000 & -9.44100000 & -6.36700000 \\
\hline v & -9.91100000 & -5.87600000 & 1.45800000 & $\mathrm{H}$ & -7.45600000 & -10.25900000 & -6.04500000 \\
\hline $\mathrm{H}$ & -10.92900000 & -5.96400000 & 1.36100000 & $\mathrm{H}$ & -5.92300000 & -9.88900000 & -6.83800000 \\
\hline & -9.24100000 & -4.79200000 & 2.01000000 & C & -7.54300000 & -8.60300000 & -7.44000000 \\
\hline$c$ & -8.99800000 & -6.69200000 & 0.89700000 & 0 & -8.48500000 & -9.22200000 & -8.05900000 \\
\hline $\mathrm{H}$ & -9.24800000 & -7.58400000 & 0.34700000 & 0 & -7.18700000 & -7.42800000 & -7.63800000 \\
\hline $\mathrm{N}$ & -7.77000000 & -6.22200000 & 1.07100000 & $\mathrm{Fe}$ & -6.25300000 & -6.20400000 & -0.44300000 \\
\hline & -7.91700000 & -5.04100000 & 1.77700000 & 0 & -4.97700000 & -7.71400000 & -0.81700000 \\
\hline $\mathrm{H}$ & -7.05900000 & -4.44500000 & 2.04100000 & $\mathrm{O}$ & -3.60700000 & -7.27800000 & -1.14200000 \\
\hline & -7.25200000 & -2.11000000 & -0.51600000 & & & & \\
\hline & -6.98000000 & -2.30800000 & 0.52300000 & \multicolumn{2}{|c|}{${ }^{5} \mathrm{TS} 3 \mathrm{C}$} & & \\
\hline $\mathrm{H}$ & -8.34100000 & -2.15100000 & -0.59300000 & \multicolumn{2}{|c|}{-4312.056517 (a.u.) } & & \\
\hline & -6.70000000 & -3.21400000 & -1.43400000 & C & -3.00100000 & -5.00200000 & 4.42400000 \\
\hline $\mathrm{O}$ & -6.73300000 & -3.07600000 & -2.66800000 & $\mathrm{H}$ & -3.61000000 & -5.69800000 & 5.00200000 \\
\hline & -6.28300000 & -4.24200000 & -0.79800000 & $\mathrm{H}$ & -3.16200000 & -4.01300000 & 4.87100000 \\
\hline N & -9.34800000 & -6.15800000 & -6.51300000 & $\mathrm{~N}$ & -3.14000000 & -4.10800000 & 2.01100000 \\
\hline & -8.46700000 & -5.63700000 & -6.67900000 & $\mathrm{H}$ & -2.34600000 & -3.46600000 & 1.97400000 \\
\hline נr & -9.68100000 & -6.44700000 & -7.43800000 & C & -3.48300000 & -5.02000000 & 3.00600000 \\
\hline$\pi$ & -9.12600000 & -6.99000000 & -5.93500000 & C & -3.95800000 & -4.32600000 & 0.95100000 \\
\hline 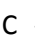 & -10.23200000 & -5.15700000 & -5.83600000 & $\mathrm{H}$ & -3.91200000 & -3.75800000 & 0.03700000 \\
\hline & -11.22400000 & -5.22200000 & -6.27700000 & $\mathrm{~N}$ & -4.80800000 & -5.31000000 & 1.19300000 \\
\hline 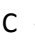 & -10.25400000 & -5.45600000 & -4.33700000 & C & -4.51100000 & -5.75200000 & 2.46300000 \\
\hline & -10.61800000 & -6.47500000 & -4.17100000 & $\mathrm{H}$ & -5.04400000 & -6.57400000 & 2.91500000 \\
\hline & -10.97800000 & -4.78000000 & -3.88000000 & C & -1.70300000 & -10.12000000 & -0.23300000 \\
\hline
\end{tabular}




\begin{tabular}{|c|c|c|c|c|c|c|c|}
\hline $\mathrm{H}$ & -1.15200000 & -11.05200000 & -0.38100000 & $\mathrm{~N}$ & -7.53800000 & -6.72000000 & -2.18000000 \\
\hline $\mathrm{H}$ & -2.29900000 & -9.94600000 & -1.13500000 & C & -6.65500000 & -7.09000000 & -3.28800000 \\
\hline & -0.92000000 & -7.72300000 & 0.30300000 & $\mathrm{H}$ & -5.93500000 & -7.77700000 & -2.84900000 \\
\hline & -1.83400000 & -7.33100000 & 0.48900000 & C & -5.78800000 & -5.92800000 & -3.88400000 \\
\hline & -0.67400000 & -9.04800000 & -0.01700000 & $\mathrm{H}$ & -6.01600000 & -4.98700000 & -3.38500000 \\
\hline & 0.26900000 & -7.07800000 & 0.37500000 & $\mathrm{H}$ & -5.94800000 & -5.79200000 & -4.95900000 \\
\hline & 0.35400000 & -6.02200000 & 0.59700000 & $S$ & -3.98900000 & -6.27800000 & -3.76400000 \\
\hline & 1.27800000 & -7.89500000 & 0.11800000 & $S$ & -3.30700000 & -6.34100000 & -1.70900000 \\
\hline & 0.69700000 & -9.12200000 & -0.12700000 & C & -7.28300000 & -7.93700000 & -4.41400000 \\
\hline - & 1.28200000 & -9.99900000 & -0.36500000 & 0 & -8.51700000 & -8.01300000 & -4.61600000 \\
\hline & -9.88100000 & -3.59700000 & 2.70000000 & $\mathrm{~N}$ & -6.41100000 & -8.60200000 & -5.19300000 \\
\hline$H$ & -10.44900000 & -2.94800000 & 2.02000000 & $\mathrm{H}$ & -5.41300000 & -8.59600000 & -4.97200000 \\
\hline-1 & -9.08000000 & -2.98300000 & 3.12500000 & C & -6.85700000 & -9.40200000 & -6.32300000 \\
\hline $\mathrm{N}$ & -9.90600000 & -5.84100000 & 1.43700000 & $\mathrm{H}$ & -7.51700000 & -10.21000000 & -5.98900000 \\
\hline$H$ & -10.92400000 & -5.93400000 & 1.34200000 & $\mathrm{H}$ & -5.97400000 & -9.86400000 & -6.77100000 \\
\hline & -9.24400000 & -4.75200000 & 1.98800000 & C & -7.58300000 & -8.59000000 & -7.42500000 \\
\hline E & -8.99100000 & -6.64800000 & 0.87000000 & $\mathrm{O}$ & -8.51400000 & -9.22700000 & -8.04300000 \\
\hline - & -9.23300000 & -7.54300000 & 0.32200000 & $\mathrm{O}$ & -7.23000000 & -7.41700000 & -7.64300000 \\
\hline $\mathrm{N}$ & -7.76500000 & -6.16900000 & 1.04300000 & $\mathrm{Fe}$ & -6.26600000 & -6.02300000 & -0.46300000 \\
\hline c & -7.91900000 & -4.98900000 & 1.75200000 & 0 & -4.81600000 & -7.40700000 & -1.11000000 \\
\hline $\mathrm{H}$ & -7.06300000 & -4.39500000 & 2.02800000 & $\mathrm{O}$ & -3.57600000 & -7.43400000 & -0.25100000 \\
\hline & -7.20200000 & -2.13000000 & -0.56300000 & & & & \\
\hline-1 & -6.90000000 & -2.38200000 & 0.45400000 & \multicolumn{2}{|c|}{${ }^{5} \mathrm{IM} 3 \mathrm{C}$} & & \\
\hline $\mathrm{H}$ & -8.29100000 & -2.18900000 & -0.62600000 & \multicolumn{2}{|c|}{-4312.226052 (a.u.) } & & \\
\hline C & -6.63800000 & -3.13400000 & -1.58200000 & C & -3.02500000 & -4.98200000 & 4.45700000 \\
\hline 0 & -6.79400000 & -2.92800000 & -2.79300000 & $\mathrm{H}$ & -3.63500000 & -5.67000000 & 5.04500000 \\
\hline 0 & -6.05300000 & -4.16000000 & -1.07300000 & $\mathrm{H}$ & -3.19200000 & -3.98800000 & 4.88800000 \\
\hline $\mathrm{N}$ & -9.35100000 & -6.11000000 & -6.51000000 & $\mathrm{~N}$ & -3.16900000 & -4.10200000 & 2.04600000 \\
\hline $\mathrm{H}$ & -8.46600000 & -5.59300000 & -6.66600000 & $\mathrm{H}$ & -2.37900000 & -3.45400000 & 2.01100000 \\
\hline $\mathrm{H}$ & -9.67700000 & -6.38800000 & -7.44100000 & C & -3.50200000 & -5.01900000 & 3.03800000 \\
\hline $\mathrm{H}$ & -9.14200000 & -6.94600000 & -5.93500000 & C & -3.96100000 & -4.34600000 & 0.97500000 \\
\hline C & -10.23300000 & -5.10800000 & -5.83300000 & $\mathrm{H}$ & -3.92200000 & -3.76700000 & 0.06700000 \\
\hline $\mathrm{H}$ & -11.22700000 & -5.17900000 & -6.26700000 & $\mathrm{~N}$ & -4.79000000 & -5.35400000 & 1.20400000 \\
\hline C & -10.24700000 & -5.39700000 & -4.33300000 & C & -4.50300000 & -5.78100000 & 2.48400000 \\
\hline $\mathrm{H}$ & -10.60600000 & -6.41700000 & -4.16000000 & $\mathrm{H}$ & -5.02900000 & -6.60800000 & 2.93500000 \\
\hline $\mathrm{H}$ & -10.97300000 & -4.72100000 & -3.87800000 & C & -1.44300000 & -9.98100000 & -0.32600000 \\
\hline$c$ & -8.84500000 & -5.20900000 & -3.72900000 & $\mathrm{H}$ & -0.96700000 & -10.95800000 & -0.43700000 \\
\hline $\mathrm{H}$ & -8.68200000 & -4.15400000 & -3.48700000 & $\mathrm{H}$ & -1.93200000 & -9.74200000 & -1.27500000 \\
\hline $\mathrm{H}$ & -8.08000000 & -5.44000000 & -4.46400000 & $\mathrm{~N}$ & -0.47100000 & -7.62400000 & -0.11300000 \\
\hline C & -8.65900000 & -5.98700000 & -2.43000000 & $\mathrm{H}$ & -1.21400000 & -7.14300000 & -0.63400000 \\
\hline 0 & -9.57800000 & -5.83500000 & -1.59500000 & C & -0.36400000 & -8.99400000 & 0.02900000 \\
\hline C & -9.63700000 & -3.69500000 & -6.10400000 & C & 0.66700000 & -7.06500000 & 0.35700000 \\
\hline 0 & -8.43400000 & -3.67300000 & -6.50600000 & $\mathrm{H}$ & 0.84600000 & -5.99400000 & 0.33600000 \\
\hline & -10.41200000 & -2.75900000 & -5.83800000 & $\mathrm{~N}$ & 1.51100000 & -7.98200000 & 0.80700000 \\
\hline
\end{tabular}




\begin{tabular}{|c|c|c|c|c|c|c|c|}
\hline & 0.87300000 & -9.18900000 & 0.60200000 & C & -7.30300000 & -7.96600000 & -4.43300000 \\
\hline$H$ & 1.33300000 & -10.12800000 & 0.87200000 & 0 & -8.53800000 & -8.05300000 & -4.62100000 \\
\hline & -9.89300000 & -3.51200000 & 2.73800000 & $\mathrm{~N}$ & -6.43400000 & -8.64500000 & -5.20200000 \\
\hline & -10.45500000 & -2.85000000 & 2.06600000 & $\mathrm{H}$ & -5.42900000 & -8.59700000 & -5.02600000 \\
\hline & -9.09300000 & -2.90800000 & 3.17900000 & $\mathrm{C}$ & -6.88300000 & -9.43600000 & -6.33700000 \\
\hline & -9.91700000 & -5.74500000 & 1.45600000 & $\mathrm{H}$ & -7.54300000 & -10.24500000 & -6.00800000 \\
\hline & -10.93500000 & -5.84900000 & 1.37200000 & $\mathrm{H}$ & -6.00100000 & -9.89600000 & -6.79100000 \\
\hline & -9.25500000 & -4.65700000 & 2.01000000 & C & -7.60900000 & -8.61100000 & -7.42800000 \\
\hline & -9.00500000 & -6.54800000 & 0.88100000 & 0 & -8.53800000 & -9.24100000 & -8.05700000 \\
\hline & -9.25100000 & -7.43900000 & 0.33100000 & $\mathrm{O}$ & -7.25900000 & -7.43300000 & -7.62800000 \\
\hline & -7.77900000 & -6.06400000 & 1.04200000 & $\mathrm{Fe}$ & -6.32200000 & -6.02700000 & -0.48800000 \\
\hline & -7.93000000 & -4.88800000 & 1.76000000 & $\mathrm{O}$ & -4.62600000 & -7.07500000 & -1.21200000 \\
\hline & -7.07300000 & -4.29400000 & 2.03300000 & $\mathrm{O}$ & -2.23400000 & -6.72000000 & -1.89200000 \\
\hline & -7.18300000 & -2.16400000 & -0.59200000 & & & & \\
\hline & -6.86400000 & -2.42900000 & 0.41700000 & \multicolumn{4}{|c|}{${ }^{5} \mathrm{TS} 2 \mathrm{~b}^{\prime}$} \\
\hline & -8.27300000 & -2.22800000 & -0.63900000 & \multicolumn{4}{|c|}{-4312.118428 (a.u.) } \\
\hline & -6.63300000 & -3.15100000 & -1.63400000 & C & -3.02400000 & -4.94600000 & 4.39900000 \\
\hline & -6.81800000 & -2.93400000 & -2.83800000 & $\mathrm{H}$ & -3.59400000 & -5.63700000 & 5.02100000 \\
\hline & -6.02600000 & -4.18200000 & -1.15300000 & $\mathrm{H}$ & -3.19700000 & -3.94600000 & 4.81500000 \\
\hline & -9.41400000 & -6.15100000 & -6.52100000 & $\mathrm{~N}$ & -3.32800000 & -4.06700000 & 2.01600000 \\
\hline & -8.52900000 & -5.63600000 & -6.68800000 & $\mathrm{H}$ & -2.60300000 & -3.35300000 & 1.98600000 \\
\hline & -9.74400000 & -6.43900000 & -7.44700000 & C & -3.53900000 & -5.02700000 & 2.99700000 \\
\hline & -9.19900000 & -6.98200000 & -5.93900000 & C & -4.11500000 & -4.38300000 & 0.95600000 \\
\hline & -10.29300000 & -5.14200000 & -5.84900000 & $\mathrm{H}$ & -4.16100000 & -3.78500000 & 0.06200000 \\
\hline & -11.28500000 & -5.20600000 & -6.28900000 & $\mathrm{~N}$ & -4.81700000 & -5.48100000 & 1.18200000 \\
\hline & -10.31500000 & -5.42700000 & -4.34700000 & C & -4.45700000 & -5.89100000 & 2.44800000 \\
\hline & -10.67700000 & -6.44500000 & -4.16900000 & $\mathrm{H}$ & -4.86900000 & -6.78400000 & 2.89200000 \\
\hline & -11.03900000 & -4.74500000 & -3.89700000 & C & -1.63700000 & -10.12700000 & -0.37600000 \\
\hline & -8.91000000 & -5.23700000 & -3.75200000 & $\mathrm{H}$ & -1.08500000 & -11.06800000 & -0.39100000 \\
\hline & -8.74700000 & -4.18100000 & -3.51100000 & $\mathrm{H}$ & -2.20500000 & -10.06500000 & -1.31100000 \\
\hline & -8.15600000 & -5.46100000 & -4.49800000 & $\mathrm{~N}$ & -0.92800000 & -7.67500000 & -0.27300000 \\
\hline & -8.69200000 & -6.01400000 & -2.45900000 & $\mathrm{H}$ & -1.87000000 & -7.30700000 & -0.40900000 \\
\hline & -9.60300000 & -5.88300000 & -1.61100000 & C & -0.62800000 & -9.02400000 & -0.24600000 \\
\hline & -9.68600000 & -3.73200000 & -6.11700000 & C & 0.22500000 & -6.98700000 & -0.09000000 \\
\hline & -8.48400000 & -3.71700000 & -6.51900000 & $\mathrm{H}$ & 0.27100000 & -5.90400000 & -0.06800000 \\
\hline & -10.45500000 & -2.79200000 & -5.84600000 & $\mathrm{~N}$ & 1.25900000 & -7.80200000 & 0.04500000 \\
\hline & -7.54500000 & -6.71100000 & -2.22500000 & C & 0.73500000 & -9.07500000 & -0.05600000 \\
\hline & -6.67400000 & -7.07000000 & -3.34900000 & $\mathrm{H}$ & 1.35100000 & -9.95900000 & 0.01700000 \\
\hline & -5.90500000 & -7.69400000 & -2.90700000 & C & -9.76900000 & -3.60100000 & 2.67800000 \\
\hline & -5.88900000 & -5.89200000 & -4.02000000 & $\mathrm{H}$ & -10.29100000 & -2.88900000 & \\
\hline & -6.16600000 & -4.94200000 & -3.56400000 & $\mathrm{H}$ & -8.93400000 & -3.05800000 & 3.13300000 \\
\hline & -6.07700000 & -5.81300000 & -5.09500000 & $\mathrm{~N}$ & -9.97100000 & -5.73300000 & 1.27700000 \\
\hline & -4.06400000 & -6.05700000 & -3.93600000 & $\mathrm{H}$ & -10.99700000 & -5.74400000 & 1.21700000 \\
\hline & -3.58100000 & -6.05200000 & -1.76900000 & C & -9.21100000 & -4.76300000 & 1.91100000 \\
\hline
\end{tabular}




\begin{tabular}{|c|c|c|c|c|c|c|c|}
\hline 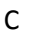 & -9.13400000 & -6.61000000 & 0.68700000 & 0 & -8.24900000 & -9.23600000 & -8.29800000 \\
\hline $\mathrm{H}$ & -9.46500000 & -7.43800000 & 0.08500000 & $\mathrm{O}$ & -7.15400000 & -7.36300000 & -7.69000000 \\
\hline $\mathrm{v}$ & -7.86700000 & -6.26100000 & 0.88900000 & $\mathrm{Fe}$ & -6.33700000 & -6.46000000 & -0.63800000 \\
\hline & -7.91200000 & -5.11700000 & 1.66800000 & $\mathrm{O}$ & -3.45600000 & -7.19600000 & -1.27700000 \\
\hline+ & -7.00400000 & -4.62200000 & 1.96900000 & $\mathrm{O}$ & -5.05300000 & -7.74100000 & -0.87600000 \\
\hline & -7.23300000 & -2.27600000 & -0.59700000 & & & & \\
\hline 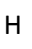 & -6.93400000 & -2.50400000 & 0.42800000 & \multicolumn{4}{|l|}{${ }^{3} \mathrm{Ra}$} \\
\hline-1 & -8.32800000 & -2.30700000 & -0.63900000 & \multicolumn{4}{|c|}{-4312.098457 (a.u.) } \\
\hline E & -6.74400000 & -3.37200000 & -1.56500000 & C & -3.18100000 & -5.00300000 & 4.16600000 \\
\hline 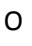 & -6.75200000 & -3.16800000 & -2.78900000 & $\mathrm{H}$ & -3.76000000 & -5.68700000 & 4.78800000 \\
\hline 0 & -6.44400000 & -4.47400000 & -0.98600000 & H & -3.40300000 & -3.99200000 & 4.53000000 \\
\hline 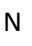 & -9.22300000 & -6.18200000 & -6.43700000 & $\mathrm{~N}$ & -3.26400000 & -4.31800000 & 1.70000000 \\
\hline H & -8.36000000 & -5.62600000 & -6.58800000 & $\mathrm{H}$ & -2.53500000 & -3.60500000 & 1.69200000 \\
\hline-1 & -9.52500000 & -6.47100000 & -7.37300000 & C & -3.62900000 & -5.16000000 & 2.74500000 \\
\hline H & -8.94800000 & -7.02200000 & -5.88500000 & C & -3.99600000 & -4.64300000 & 0.61700000 \\
\hline$c$ & -10.15100000 & -5.22000000 & -5.77200000 & $\mathrm{H}$ & -3.91300000 & -4.15200000 & -0.33700000 \\
\hline $\mathrm{H}$ & -11.11700000 & -5.27700000 & -6.26700000 & $\mathrm{~N}$ & -4.82200000 & -5.64600000 & 0.89400000 \\
\hline$c$ & -10.28700000 & -5.54500000 & -4.28200000 & C & -4.59200000 & -5.98600000 & 2.21100000 \\
\hline $\mathrm{H}$ & -10.79900000 & -6.50100000 & -4.14400000 & H & -5.10800000 & -6.80900000 & 2.67800000 \\
\hline $\mathrm{H}$ & -10.95300000 & -4.77100000 & -3.88700000 & C & -1.74200000 & -10.07000000 & -0.49100000 \\
\hline C & -8.92600000 & -5.55300000 & -3.55500000 & $\mathrm{H}$ & -1.16300000 & -10.99500000 & -0.49800000 \\
\hline $\mathrm{H}$ & -8.86100000 & -4.70600000 & -2.86200000 & $\mathrm{H}$ & -2.33100000 & -10.04700000 & -1.41300000 \\
\hline $\mathrm{H}$ & -8.11300000 & -5.35600000 & -4.25000000 & $\mathrm{~N}$ & -1.06100000 & -7.59400000 & -0.52700000 \\
\hline C & -8.64200000 & -6.78500000 & -2.69500000 & $\mathrm{H}$ & -1.98900000 & -7.20600000 & -0.71100000 \\
\hline $\mathrm{O}$ & -9.62600000 & -7.33700000 & -2.14300000 & C & -0.75600000 & -8.94100000 & -0.41500000 \\
\hline C & -9.56300000 & -3.78700000 & -5.97800000 & C & 0.08800000 & -6.89200000 & -0.36000000 \\
\hline $\mathrm{O}$ & -8.35400000 & -3.74100000 & -6.35800000 & $\mathrm{H}$ & 0.12900000 & -5.81000000 & -0.39500000 \\
\hline $\mathrm{O}$ & -10.35600000 & -2.86600000 & -5.70800000 & $\mathrm{~N}$ & 1.12100000 & -7.69300000 & -0.15800000 \\
\hline $\mathrm{N}$ & -7.36100000 & -7.15600000 & -2.40800000 & C & 0.60300000 & -8.97100000 & -0.19700000 \\
\hline C & -6.38400000 & -7.26600000 & -3.49700000 & $\mathrm{H}$ & 1.22200000 & -9.84800000 & -0.06300000 \\
\hline $\mathrm{H}$ & -5.56600000 & -7.83400000 & -3.05900000 & C & -9.75200000 & -3.52300000 & 2.60500000 \\
\hline $\mathrm{C}$ & -5.74000000 & -5.95000000 & -4.04900000 & $\mathrm{H}$ & -10.27700000 & -2.80500000 & 1.96000000 \\
\hline $\mathrm{H}$ & -6.12800000 & -5.06500000 & -3.54200000 & H & -8.93600000 & & 3.08400000 \\
\hline $\mathrm{H}$ & -5.89600000 & -5.82200000 & -5.12600000 & $\mathrm{~N}$ & -9.85200000 & -5.71900000 & 1.27700000 \\
\hline 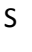 & -3.89800000 & -5.89700000 & -3.89900000 & $\mathrm{H}$ & -10.87100000 & -5.83500000 & 1.30400000 \\
\hline 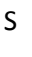 & -3.51100000 & -5.69800000 & -1.87500000 & C & -9.15300000 & -4.64700000 & 1.80800000 \\
\hline C & -6.93600000 & -8.09900000 & -4.66500000 & C & -8.98900000 & -6.49200000 & 0.58600000 \\
\hline 0 & -8.16600000 & -8.17400000 & -4.89300000 & $\mathrm{H}$ & -9.27700000 & -7.38200000 & 0.05800000 \\
\hline $\mathrm{N}$ & -6.06800000 & -8.71700000 & -5.46800000 & $\mathrm{~N}$ & -7.77200000 & -5.96700000 & 0.60400000 \\
\hline $\mathrm{H}$ & -5.06400000 & -8.69700000 & -5.26500000 & C & -7.86000000 & -4.83700000 & 1.39600000 \\
\hline C & -6.55300000 & -9.44900000 & -6.63300000 & $\mathrm{H}$ & -6.98900000 & -4.24300000 & 1.61400000 \\
\hline $\mathrm{H}$ & -7.16100000 & -10.30300000 & -6.31800000 & C & -7.07400000 & -2.35100000 & -0.62300000 \\
\hline $\mathrm{H}$ & -5.68400000 & -9.83700000 & -7.16900000 & $\mathrm{H}$ & -6.71400000 & -2.57000000 & 0.38100000 \\
\hline 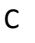 & -7.38900000 & -8.58900000 & -7.61400000 & $\mathrm{H}$ & -8.16700000 & -2.40300000 & -0.61000000 \\
\hline
\end{tabular}




\begin{tabular}{|c|c|c|c|c|c|c|c|}
\hline C & -6.61600000 & -3.42200000 & -1.63700000 & C & -3.03500000 & -4.96200000 & 4.29400000 \\
\hline 0 & -6.81400000 & -3.22000000 & -2.84400000 & $\mathrm{H}$ & -3.62500000 & -5.61100000 & 4.94300000 \\
\hline 0 & -6.10900000 & -4.47900000 & -1.11700000 & $\mathrm{H}$ & -3.22800000 & -3.93400000 & 4.62200000 \\
\hline $\mathrm{N}$ & -9.20300000 & -6.18200000 & -6.38500000 & $\mathrm{~N}$ & -3.13700000 & -4.37600000 & 1.79400000 \\
\hline $\mathrm{H}$ & -8.34200000 & -5.62300000 & -6.53200000 & $\mathrm{H}$ & -2.43600000 & -3.63600000 & 1.75300000 \\
\hline $\mathrm{H}$ & -9.51900000 & -6.44500000 & -7.32400000 & C & -3.48500000 & -5.17000000 & 2.88100000 \\
\hline $\mathrm{H}$ & -8.92700000 & -7.03300000 & -5.85200000 & C & -3.85400000 & -4.80100000 & 0.72800000 \\
\hline C & -10.11800000 & -5.24100000 & -5.67400000 & $\mathrm{H}$ & -3.78500000 & -4.34400000 & -0.24700000 \\
\hline $\mathrm{H}$ & -11.10400000 & -5.31100000 & -6.12600000 & $\mathrm{~N}$ & -4.64100000 & -5.81700000 & 1.04800000 \\
\hline C & -10.18000000 & -5.59700000 & -4.18500000 & C & -4.41000000 & -6.06000000 & 2.38400000 \\
\hline $\mathrm{H}$ & -10.63800000 & -6.58100000 & -4.05000000 & $\mathrm{H}$ & -4.90200000 & -6.86400000 & 2.90700000 \\
\hline $\mathrm{H}$ & -10.86800000 & -4.86700000 & -3.74800000 & C & -1.65500000 & -10.08600000 & -0.40700000 \\
\hline C & -8.79500000 & -5.55100000 & -3.50900000 & $\mathrm{H}$ & -1.07700000 & -11.01200000 & -0.43100000 \\
\hline $\mathrm{H}$ & -8.70300000 & -4.65100000 & -2.89300000 & $\mathrm{H}$ & -2.26100000 & -10.05200000 & -1.31900000 \\
\hline $\mathrm{H}$ & -8.01300000 & -5.40500000 & -4.24900000 & $\mathrm{~N}$ & -0.99800000 & -7.61100000 & -0.43000000 \\
\hline C & -8.46500000 & -6.72000000 & -2.58100000 & $\mathrm{H}$ & -1.92300000 & -7.24300000 & -0.64000000 \\
\hline 0 & -9.42800000 & -7.27800000 & -2.01700000 & C & -0.67700000 & -8.95400000 & -0.32600000 \\
\hline C & -9.55900000 & -3.79900000 & -5.89600000 & C & 0.14000000 & -6.89300000 & -0.24100000 \\
\hline 0 & -8.35700000 & -3.73400000 & -6.29300000 & $\mathrm{H}$ & 0.17000000 & -5.81100000 & -0.26800000 \\
\hline 0 & -10.36500000 & -2.88800000 & -5.62700000 & $\mathrm{~N}$ & 1.17800000 & -7.68400000 & -0.03000000 \\
\hline $\mathrm{N}$ & -7.15100000 & -7.04400000 & -2.30700000 & C & 0.67900000 & -8.96900000 & -0.08900000 \\
\hline C & -6.29100000 & -7.24400000 & -3.48100000 & $\mathrm{H}$ & 1.30700000 & -9.83900000 & 0.04500000 \\
\hline $\mathrm{H}$ & -5.49000000 & -7.89400000 & -3.11700000 & C & -9.73800000 & -3.74300000 & 2.59900000 \\
\hline C & -5.58300000 & -6.00000000 & -4.07100000 & $\mathrm{H}$ & -10.25300000 & -3.05400000 & 1.91700000 \\
\hline $\mathrm{H}$ & -6.14200000 & -5.09200000 & -3.85400000 & $\mathrm{H}$ & -8.91000000 & -3.18200000 & 3.04300000 \\
\hline $\mathrm{H}$ & -5.43100000 & -6.09800000 & -5.14900000 & $\mathrm{~N}$ & -9.91300000 & -5.92300000 & 1.26300000 \\
\hline S & -3.91000000 & -5.70900000 & -3.30200000 & $\mathrm{H}$ & -10.93800000 & -5.94400000 & 1.21500000 \\
\hline $\mathrm{s}$ & -4.06600000 & -6.92900000 & -1.61500000 & C & -9.16500000 & -4.92500000 & 1.86900000 \\
\hline C & -6.93600000 & -8.06300000 & -4.60700000 & C & -9.06700000 & -6.79100000 & 0.67100000 \\
\hline 0 & -8.17600000 & -8.13400000 & -4.77300000 & $\Pi$ & -9.39100000 & -7.63300000 & 0.08500000 \\
\hline $\mathrm{N}$ & -6.11000000 & -8.71300000 & -5.43400000 & $\mathrm{~N}$ & -7.80600000 & -6.40500000 & 0.83800000 \\
\hline $\mathrm{H}$ & -5.09700000 & -8.66600000 & -5.29400000 & C & -7.86200000 & -5.25300000 & 1.60500000 \\
\hline C & -6.64600000 & -9.45800000 & -6.56300000 & $\mathrm{H}$ & -6.96300000 & -4.72800000 & 1.88300000 \\
\hline $\mathrm{H}$ & -7.30600000 & -10.25700000 & -6.21100000 & C & -7.12300000 & -2.45300000 & -0.50000000 \\
\hline $\mathrm{H}$ & -5.80900000 & -9.92700000 & -7.08600000 & 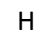 & -6.82200000 & -2.62000000 & 0.53400000 \\
\hline C & -7.43200000 & -8.59100000 & -7.57900000 & $\mathrm{H}$ & -8.21900000 & -2.49500000 & -0.54100000 \\
\hline 0 & -8.29300000 & -9.22500000 & -8.27400000 & C & -6.63600000 & -3.61000000 & -1.40300000 \\
\hline $\mathrm{O}$ & -7.16500000 & -7.37200000 & -7.66300000 & $\mathrm{O}$ & -6.59300000 & -3.46600000 & -2.63100000 \\
\hline $\mathrm{Fe}$ & -6.08800000 & -6.38600000 & -0.60100000 & $\mathrm{O}$ & -6.41600000 & -4.68500000 & -0.73400000 \\
\hline $\mathrm{O}$ & -5.87100000 & -9.14600000 & -0.40500000 & $\mathrm{~N}$ & -9.22400000 & -6.17200000 & -6.50000000 \\
\hline \multirow[t]{2}{*}{0} & -6.04200000 & -8.05400000 & 0.30000000 & $\mathrm{H}$ & -8.34500000 & -5.63800000 & -6.62700000 \\
\hline & & & & $\mathrm{H}$ & -9.52900000 & -6.41300000 & -7.45000000 \\
\hline \multicolumn{4}{|c|}{ TS1a } & $\mathrm{H}$ & -8.97600000 & -7.04100000 & -5.98200000 \\
\hline \multicolumn{4}{|c|}{-4312.073626 (a.u.) } & C & -10.12600000 & -5.21700000 & -5.79300000 \\
\hline
\end{tabular}




\begin{tabular}{|c|c|c|c|c|c|c|c|}
\hline $\mathrm{H}$ & -11.10200000 & -5.25200000 & -6.27200000 & $\mathrm{~N}$ & -4.82300000 & -5.46500000 & 1.16200000 \\
\hline C & -10.23300000 & -5.58800000 & -4.30900000 & C & -4.52400000 & -5.93000000 & 2.42500000 \\
\hline $\mathrm{H}$ & -10.73700000 & -6.55000000 & -4.19300000 & $\mathrm{H}$ & -5.00400000 & -6.80900000 & 2.82500000 \\
\hline $\mathrm{H}$ & -10.89600000 & -4.83200000 & -3.87700000 & C & -1.45300000 & -10.41900000 & -0.46000000 \\
\hline C & -8.85800000 & -5.60200000 & -3.61200000 & $\mathrm{H}$ & -0.85500000 & -11.30400000 & -0.24300000 \\
\hline $\mathrm{H}$ & -8.74800000 & -4.72300000 & -2.96700000 & $\mathrm{H}$ & -1.96700000 & -10.61000000 & -1.40800000 \\
\hline $\mathrm{H}$ & -8.05700000 & -5.47000000 & -4.33700000 & $\mathrm{~N}$ & -0.89800000 & -8.07500000 & -1.19200000 \\
\hline C & -8.57400000 & -6.80100000 & -2.70500000 & $\mathrm{H}$ & -1.88100000 & -7.90900000 & -1.45800000 \\
\hline $\mathrm{O}$ & -9.54100000 & -7.32600000 & -2.10900000 & C & -0.51800000 & -9.25900000 & -0.59600000 \\
\hline C & -9.52400000 & -3.78700000 & -5.96700000 & C & 0.17500000 & -7.24200000 & -1.17500000 \\
\hline $\mathrm{O}$ & -8.31200000 & -3.74400000 & -6.33600000 & $\mathrm{H}$ & 0.14100000 & -6.23100000 & -1.55800000 \\
\hline 0 & -10.31000000 & -2.86400000 & -5.68100000 & $\mathrm{~N}$ & 1.22900000 & -7.81900000 & -0.62600000 \\
\hline$N$ & -7.28400000 & -7.18000000 & -2.46200000 & C & 0.80600000 & -9.08000000 & -0.26200000 \\
\hline $\mathrm{C}$ & -6.37900000 & -7.33800000 & -3.59400000 & $\mathrm{H}$ & 1.46400000 & -9.79200000 & 0.21600000 \\
\hline $\mathrm{H}$ & -5.60700000 & -8.01600000 & -3.22200000 & C & -9.83700000 & -3.42100000 & 2.68300000 \\
\hline C & -5.64000000 & -6.05000000 & -4.09600000 & $\mathrm{H}$ & -10.38300000 & -2.72500000 & 2.03200000 \\
\hline $\mathrm{H}$ & -6.05500000 & -5.16300000 & -3.62100000 & $\mathrm{H}$ & -9.04200000 & -2.84100000 & 3.16500000 \\
\hline $\mathrm{H}$ & -5.67600000 & -5.94400000 & -5.18500000 & $\mathrm{~N}$ & -9.83100000 & -5.61600000 & 1.33300000 \\
\hline $\mathrm{s}$ & -3.85500000 & -6.12600000 & -3.65000000 & $\mathrm{H}$ & -10.84500000 & -5.77300000 & 1.31200000 \\
\hline $\mathrm{s}$ & -4.04600000 & -7.05300000 & -1.74700000 & C & -9.18800000 & -4.52400000 & 1.90100000 \\
\hline C & -6.98000000 & -8.11200000 & -4.77200000 & $\mathrm{C}$ & -8.91600000 & -6.35200000 & 0.67400000 \\
\hline $\mathrm{O}$ & -8.21400000 & -8.19600000 & -4.97200000 & $\mathrm{H}$ & -9.14400000 & -7.25100000 & 0.12500000 \\
\hline$N$ & -6.11800000 & -8.70600000 & -5.60200000 & $\mathrm{~N}$ & -7.71600000 & -5.80700000 & 0.79300000 \\
\hline $\mathrm{H}$ & -5.11000000 & -8.65600000 & -5.43600000 & C & -7.87000000 & -4.67800000 & 1.56600000 \\
\hline C & -6.61300000 & -9.45000000 & -6.75200000 & $\mathrm{H}$ & -7.02800000 & -4.06000000 & 1.82200000 \\
\hline $\mathrm{H}$ & -7.21600000 & -10.30000000 & -6.41900000 & C & -7.19400000 & -2.25300000 & -0.72200000 \\
\hline $\mathrm{H}$ & -5.74700000 & -9.84300000 & -7.28900000 & $\mathrm{H}$ & -7.00900000 & -2.43800000 & 0.33500000 \\
\hline C & -7.45400000 & -8.60300000 & -7.73800000 & $\mathrm{H}$ & -8.27000000 & -2.29700000 & -0.89800000 \\
\hline $\mathrm{O}$ & -8.30500000 & -9.26000000 & -8.42100000 & C & -6.56900000 & -3.36200000 & -1.59000000 \\
\hline $\mathrm{O}$ & -7.23100000 & -7.37400000 & -7.82000000 & 0 & -6.68400000 & -3.30500000 & -2.82200000 \\
\hline $\mathrm{Fe}$ & -6.21500000 & -6.66200000 & -0.62100000 & $\mathrm{O}$ & -5.98500000 & -4.28700000 & -0.91300000 \\
\hline 0 & -4.75700000 & -8.84200000 & -1.17100000 & $\mathrm{~N}$ & -9.44800000 & -6.49300000 & -6.71600000 \\
\hline \multirow[t]{2}{*}{$\mathrm{O}$} & -5.76000000 & -8.44400000 & -0.25500000 & $\mathrm{H}$ & -8.45400000 & -6.23600000 & -6.68400000 \\
\hline & & & & $\mathrm{H}$ & -9.70000000 & -6.39700000 & -7.71300000 \\
\hline \multicolumn{2}{|c|}{${ }^{3}$ IM1a } & & & $\mathrm{H}$ & -9.55100000 & -7.49700000 & -6.49100000 \\
\hline \multicolumn{2}{|c|}{-4312.143720 (a.u.) } & & & $\mathrm{C}$ & -10.20100000 & -5.51100000 & -5.85200000 \\
\hline C & -3.05800000 & -5.09400000 & 4.40900000 & $\mathrm{H}$ & -11.24900000 & -5.59300000 & -6.12300000 \\
\hline $\mathrm{H}$ & -3.62300000 & -5.81600000 & 5.00000000 & C & -9.99300000 & -5.84000000 & -4.36900000 \\
\hline $\mathrm{H}$ & -3.25800000 & -4.11200000 & 4.85400000 & $\mathrm{H}$ & -10.37300000 & -6.84400000 & -4.14800000 \\
\hline $\mathrm{N}$ & -3.26100000 & -4.18000000 & 2.03300000 & $\mathrm{H}$ & -10.59200000 & -5.12800000 & -3.79900000 \\
\hline $\mathrm{H}$ & -2.49000000 & -3.51300000 & 2.02200000 & C & -8.52600000 & -5.71700000 & -3.96700000 \\
\hline C & -3.55800000 & -5.13700000 & 2.99800000 & $\mathrm{H}$ & -8.19200000 & -4.68900000 & -4.13600000 \\
\hline C & -4.03300000 & -4.41900000 & 0.95400000 & $\mathrm{H}$ & -7.92300000 & -6.34800000 & -4.61500000 \\
\hline $\mathrm{H}$ & -4.00800000 & -3.82900000 & 0.05500000 & C & -8.26300000 & -5.94100000 & -2.47000000 \\
\hline
\end{tabular}




\begin{tabular}{|c|c|c|c|c|c|c|c|}
\hline 0 & -9.10100000 & -5.40800000 & -1.72000000 & C & -0.55800000 & -9.23800000 & -0.65400000 \\
\hline C & -9.67900000 & -4.07700000 & -6.17700000 & C & 0.14800000 & -7.26400000 & -1.38700000 \\
\hline 0 & -8.49500000 & -4.01300000 & -6.61800000 & $\mathrm{H}$ & 0.12900000 & -6.30800000 & -1.89200000 \\
\hline 0 & -10.48100000 & -3.16200000 & -5.91000000 & $\mathrm{~N}$ & 1.14700000 & -7.74400000 & -0.67500000 \\
\hline $\mathrm{N}$ & -7.14200000 & -6.59900000 & -2.06100000 & C & 0.71800000 & -8.97400000 & -0.21800000 \\
\hline C & -6.31900000 & -7.27800000 & -3.05800000 & $\mathrm{H}$ & 1.34600000 & -9.60500000 & 0.39500000 \\
\hline $\mathrm{H}$ & -5.59200000 & -7.88100000 & -2.50400000 & C & -9.92800000 & -3.39000000 & 2.73200000 \\
\hline C & -5.54600000 & -6.28800000 & -3.97300000 & $\mathrm{H}$ & -10.50000000 & -2.71900000 & 2.07700000 \\
\hline $\mathrm{H}$ & -6.13200000 & -5.38500000 & -4.10100000 & $\mathrm{H}$ & -9.13900000 & -2.78500000 & 3.19100000 \\
\hline $\mathrm{H}$ & -5.32600000 & -6.71200000 & -4.95400000 & $\mathrm{~N}$ & -9.91900000 & -5.59400000 & 1.40500000 \\
\hline$S$ & -3.93900000 & -5.68100000 & -3.29400000 & $\mathrm{H}$ & -10.93300000 & -5.73500000 & 1.34600000 \\
\hline $\mathrm{s}$ & -3.91700000 & -6.52000000 & -1.35600000 & C & -9.27400000 & -4.50400000 & 1.97400000 \\
\hline C & -7.08200000 & -8.36300000 & -3.86200000 & C & -8.98700000 & -6.37200000 & 0.81800000 \\
\hline 0 & -8.23200000 & -8.71800000 & -3.55900000 & $\mathrm{H}$ & -9.21300000 & -7.27700000 & 0.27800000 \\
\hline $\mathrm{N}$ & -6.40300000 & -8.91800000 & -4.89500000 & $\mathrm{~N}$ & -7.77800000 & -5.86200000 & 0.98100000 \\
\hline $\mathrm{H}$ & -5.41500000 & -8.71500000 & -5.03200000 & C & -7.94600000 & -4.70600000 & 1.70700000 \\
\hline C & -7.02500000 & -9.86200000 & -5.81200000 & $\mathrm{H}$ & -7.10200000 & -4.09400000 & 1.97900000 \\
\hline $\mathrm{H}$ & -7.71800000 & -10.50100000 & -5.26600000 & C & -7.19100000 & -2.27400000 & -0.69000000 \\
\hline $\mathrm{H}$ & -6.24300000 & -10.48300000 & -6.25500000 & $\mathrm{H}$ & -7.00300000 & -2.47400000 & 0.36400000 \\
\hline C & -7.77200000 & -9.11700000 & -6.94500000 & $\mathrm{H}$ & -8.26700000 & -2.33100000 & -0.86300000 \\
\hline 0 & -9.02600000 & -9.35800000 & -7.06400000 & $\mathrm{C}$ & -6.55500000 & -3.36300000 & -1.57200000 \\
\hline 0 & -7.13500000 & -8.29400000 & -7.64400000 & $\mathrm{O}$ & -6.58700000 & -3.25700000 & -2.80300000 \\
\hline $\mathrm{Fe}$ & -6.10800000 & -6.21700000 & -0.36600000 & 0 & -6.04400000 & -4.33600000 & -0.90300000 \\
\hline 0 & -3.59600000 & -8.03800000 & -1.55100000 & $\mathrm{~N}$ & -9.37000000 & -6.47600000 & -6.69300000 \\
\hline \multirow[t]{2}{*}{$\mathrm{O}$} & -6.04900000 & -7.77100000 & 0.13300000 & $\mathrm{H}$ & -8.37500000 & -6.22800000 & -6.65600000 \\
\hline & & & & $\mathrm{H}$ & -9.61600000 & -6.38300000 & -7.69200000 \\
\hline \multicolumn{2}{|c|}{${ }^{3}$ TS2a } & & & $\mathrm{H}$ & -9.48400000 & -7.47600000 & -6.46100000 \\
\hline \multicolumn{2}{|c|}{-4312.135115 (a.u.) } & & & $\mathrm{C}$ & -10.12800000 & -5.49800000 & -5.83100000 \\
\hline C & -3.04700000 & -5.01800000 & 4.34300000 & $\mathrm{H}$ & -11.17900000 & -5.61000000 & -6.08200000 \\
\hline $\mathrm{H}$ & -3.63700000 & -5.72400000 & 4.92900000 & $\mathrm{C}$ & -9.88900000 & -5.81400000 & -4.35300000 \\
\hline $\mathrm{H}$ & -3.23500000 & -4.02800000 & 4.77600000 & $\mathrm{H}$ & -10.18900000 & -6.84600000 & -4.13800000 \\
\hline $\mathrm{N}$ & -3.16000000 & -4.17600000 & 1.91800000 & $\mathrm{H}$ & -10.54300000 & -5.15600000 & -3.77800000 \\
\hline $\mathrm{H}$ & -2.37800000 & -3.51800000 & 1.90000000 & C & -8.43900000 & -5.58300000 & -3.92100000 \\
\hline C & -3.51900000 & -5.07000000 & 2.92300000 & $\mathrm{H}$ & -8.20100000 & -4.51700000 & -3.98400000 \\
\hline C & -3.93200000 & -4.42500000 & 0.83900000 & $\mathrm{H}$ & -7.75000000 & -6.08500000 & -4.59700000 \\
\hline $\mathrm{H}$ & -3.86700000 & -3.88700000 & -0.09100000 & C & -8.21700000 & -5.92700000 & -2.44200000 \\
\hline $\mathrm{N}$ & -4.77400000 & -5.42000000 & 1.08700000 & $\mathrm{O}$ & -9.06200000 & -5.44400000 & -1.67000000 \\
\hline C & -4.51300000 & -5.84200000 & 2.37200000 & C & -9.64300000 & -4.05800000 & -6.17400000 \\
\hline $\mathrm{H}$ & -5.04100000 & -6.67800000 & 2.80100000 & $\mathrm{O}$ & -8.46800000 & -3.97500000 & -6.63300000 \\
\hline C & -1.46200000 & -10.41600000 & -0.47800000 & 0 & -10.45700000 & -3.15600000 & -5.89700000 \\
\hline $\mathrm{H}$ & -0.83900000 & -11.28200000 & -0.25300000 & $\mathrm{~N}$ & -7.12900000 & -6.65200000 & -2.04300000 \\
\hline $\mathrm{H}$ & -1.98200000 & -10.63400000 & -1.41500000 & C & -6.27600000 & -7.28300000 & -3.04700000 \\
\hline $\mathrm{N}$ & -0.90000000 & -8.13600000 & -1.41600000 & $\mathrm{H}$ & -5.67300000 & -8.02800000 & -2.51900000 \\
\hline $\mathrm{H}$ & -1.81500000 & -7.99900000 & -1.82800000 & C & -5.31100000 & -6.28500000 & -3.75500000 \\
\hline
\end{tabular}




\begin{tabular}{|c|c|c|c|c|c|c|c|}
\hline $\mathrm{H}$ & -5.80300000 & -5.31700000 & -3.82700000 & $\mathrm{H}$ & -8.99200000 & -2.89800000 & 3.18500000 \\
\hline $\mathrm{H}$ & -5.05600000 & -6.63200000 & -4.75600000 & $\mathrm{~N}$ & -9.99000000 & -5.55800000 & 1.28900000 \\
\hline$S$ & -3.69400000 & -5.91600000 & -2.89600000 & $\mathrm{H}$ & -11.01700000 & -5.59600000 & 1.24300000 \\
\hline$C$ & -4.06600000 & -7.21200000 & -1.00900000 & $\mathrm{C}$ & -9.24000000 & -4.58000000 & 1.92100000 \\
\hline 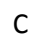 & -7.04800000 & -8.22800000 & -3.99700000 & C & -9.14600000 & -6.42000000 & 0.68800000 \\
\hline 0 & -8.22600000 & -8.54200000 & -3.77600000 & $\mathrm{H}$ & -9.47100000 & -7.24800000 & 0.08400000 \\
\hline $\mathrm{N}$ & -6.34500000 & -8.76200000 & -5.02600000 & $\mathrm{~N}$ & -7.88500000 & -6.05400000 & 0.87100000 \\
\hline $\mathrm{H}$ & -5.34100000 & -8.61500000 & -5.11000000 & C & -7.93800000 & -4.91600000 & 1.65600000 \\
\hline$C$ & -6.94900000 & -9.74400000 & -5.91900000 & $\mathrm{H}$ & -7.03600000 & -4.40800000 & 1.96000000 \\
\hline $\mathrm{H}$ & -7.61300000 & -10.39800000 & -5.35200000 & C & -7.25800000 & -2.23200000 & -0.62200000 \\
\hline H & -6.14700000 & -10.34300000 & -6.35700000 & $\mathrm{H}$ & -6.94000000 & -2.50700000 & 0.38400000 \\
\hline C & -7.73400000 & -9.05700000 & -7.05900000 & $\mathrm{H}$ & -8.35300000 & -2.24800000 & -0.64200000 \\
\hline 0 & -8.98000000 & -9.33800000 & -7.15500000 & C & -6.79500000 & -3.27900000 & -1.65700000 \\
\hline 0 & -7.12900000 & -8.23600000 & -7.79000000 & 0 & -6.87500000 & -3.01700000 & -2.86700000 \\
\hline 它 & -6.11300000 & -6.20900000 & -0.35900000 & 0 & -6.41700000 & -4.38700000 & -1.14900000 \\
\hline 0 & -3.92200000 & -8.58900000 & -1.67600000 & $\mathrm{~N}$ & -9.18500000 & -6.15500000 & -6.47700000 \\
\hline \multirow[t]{2}{*}{0} & -5.70800000 & -7.81100000 & 0.13400000 & $\mathrm{H}$ & -8.30500000 & -5.62800000 & -6.62200000 \\
\hline & & & & $\mathrm{H}$ & -9.49800000 & -6.41200000 & -7.42000000 \\
\hline \multicolumn{2}{|c|}{${ }^{3} \mathrm{IM} 2 \mathrm{a}$} & & & $\mathrm{H}$ & -8.94600000 & -7.01300000 & -5.94200000 \\
\hline \multicolumn{2}{|c|}{-4312.226299 (a.u.) } & & & $\mathrm{C}$ & -10.08200000 & -5.18100000 & -5.78600000 \\
\hline C & -3.21800000 & -4.91800000 & 4.05400000 & $\mathrm{H}$ & -11.07100000 & -5.24800000 & -6.23400000 \\
\hline $\mathrm{H}$ & -3.82900000 & -5.59700000 & 4.65300000 & C & -10.14100000 & -5.49800000 & -4.29200000 \\
\hline $\mathrm{H}$ & -3.44600000 & -3.90500000 & 4.40700000 & $\mathrm{H}$ & -10.66600000 & -6.44200000 & -4.13100000 \\
\hline $\mathrm{N}$ & -3.22800000 & -4.25300000 & 1.56400000 & $\mathrm{H}$ & -10.76500000 & -4.71400000 & -3.85300000 \\
\hline $\mathrm{H}$ & -2.47800000 & -3.56000000 & 1.55400000 & $\mathrm{C}$ & -8.74900000 & -5.54600000 & -3.63000000 \\
\hline C & -3.62100000 & -5.07100000 & 2.61700000 & $\mathrm{H}$ & -8.56700000 & -4.63200000 & -3.05500000 \\
\hline C & -3.96800000 & -4.57800000 & 0.48000000 & $\mathrm{H}$ & -7.94500000 & -5.52400000 & -4.36700000 \\
\hline $\mathrm{H}$ & -3.87300000 & -4.09900000 & -0.48100000 & $\mathrm{C}$ & -8.56100000 & -6.70500000 & -2.65600000 \\
\hline $\mathrm{N}$ & -4.81700000 & -5.55900000 & 0.76000000 & 0 & -9.59400000 & -7.13800000 & -2.08800000 \\
\hline C & -4.59700000 & -5.88100000 & & $\mathrm{C}$ & & & \\
\hline $\mathrm{H}$ & -5.14400000 & -6.67200000 & 2.57300000 & 0 & -8.31500000 & -3.70400000 & -6.45200000 \\
\hline C & -1.14400000 & -10.55400000 & -0.22900000 & 0 & -10.30500000 & -2.83200000 & -5.76200000 \\
\hline $\mathrm{H}$ & -0.44800000 & -11.32700000 & 0.08300000 & $\mathrm{~N}$ & -7.31800000 & -7.15700000 & -2.30900000 \\
\hline $\mathrm{H}$ & -1.61200000 & -10.90200000 & -1.15200000 & C & -6.35900000 & -7.50000000 & -3.35700000 \\
\hline $\mathrm{N}$ & -0.77800000 & -8.37800000 & -1.45600000 & $\mathrm{H}$ & -5.77500000 & -8.33800000 & -2.95800000 \\
\hline $\mathrm{H}$ & -1.66300000 & -8.42800000 & -1.94400000 & C & -5.32700000 & -6.40300000 & -3.74500000 \\
\hline C & -0.36700000 & -9.31400000 & -0.52300000 & $\mathrm{H}$ & -5.80200000 & -5.42000000 & -3.74800000 \\
\hline C & 0.17500000 & -7.40500000 & -1.52600000 & $\mathrm{H}$ & -4.90200000 & -6.60500000 & -4.72800000 \\
\hline $\mathrm{H}$ & 0.08500000 & -6.53900000 & -2.16900000 & $S$ & -3.92400000 & -6.28000000 & -2.55300000 \\
\hline $\mathrm{N}$ & 1.17700000 & -7.66500000 & -0.71300000 & $S$ & -4.02800000 & -8.06000000 & -1.02300000 \\
\hline C & 0.85000000 & -8.85200000 & -0.09100000 & C & -6.97700000 & -8.13600000 & -4.62900000 \\
\hline $\mathrm{H}$ & 1.50400000 & -9.31700000 & 0.63200000 & $\mathrm{O}$ & -8.21000000 & -8.16500000 & -4.83300000 \\
\hline C & -9.81500000 & -3.44300000 & 2.71300000 & $\mathrm{~N}$ & -6.14900000 & -8.72300000 & -5.50300000 \\
\hline & -10.34600000 & -2.72300000 & 2.07700000 & $\mathrm{H}$ & -5.13600000 & -8.71200000 & -5.37500000 \\
\hline
\end{tabular}




\begin{tabular}{|c|c|c|c|c|c|c|c|}
\hline C & -6.68800000 & -9.46800000 & -6.63400000 & $\mathrm{H}$ & -7.02400000 & -4.23800000 & 1.85200000 \\
\hline $\mathrm{H}$ & -7.34200000 & -10.26700000 & -6.27400000 & C & -7.06400000 & -2.34300000 & -0.61600000 \\
\hline $\mathrm{H}$ & -5.84700000 & -9.93500000 & -7.15200000 & $\mathrm{H}$ & -6.70700000 & -2.57600000 & 0.38600000 \\
\hline C & -7.47600000 & -8.61700000 & -7.65800000 & $\mathrm{H}$ & -8.15700000 & -2.40300000 & -0.61100000 \\
\hline $\mathrm{O}$ & -8.33700000 & -9.26600000 & -8.33900000 & C & -6.59200000 & -3.39700000 & -1.64200000 \\
\hline 0 & -7.21000000 & -7.40100000 & -7.76800000 & 0 & -6.80100000 & -3.18700000 & -2.84700000 \\
\hline $\mathrm{Fe}$ & -6.28000000 & -6.27400000 & -0.60500000 & $\mathrm{O}$ & -6.08000000 & -4.46300000 & -1.14500000 \\
\hline 0 & -3.67600000 & -9.30700000 & -1.81400000 & $\mathrm{~N}$ & -9.16600000 & -6.21400000 & -6.20700000 \\
\hline \multirow[t]{2}{*}{$\mathrm{O}$} & -5.48800000 & -8.16800000 & -0.55800000 & $\mathrm{H}$ & -8.32900000 & -5.62200000 & -6.38500000 \\
\hline & & & & $\mathrm{H}$ & -9.46500000 & -6.54800000 & -7.12800000 \\
\hline $\mathbf{R a}$ & & & & $\mathrm{H}$ & -8.87800000 & -7.03800000 & -5.64100000 \\
\hline 431 & 12.091969 (a.u.) & & & $\mathrm{C}$ & -10.09800000 & -5.25400000 & -5.55000000 \\
\hline C & -3.06900000 & -4.92100000 & 4.32000000 & $\mathrm{H}$ & -11.07100000 & -5.31700000 & -6.03400000 \\
\hline $\mathrm{H}$ & -3.63900000 & -5.60600000 & 4.95000000 & $\mathrm{C}$ & -10.21900000 & -5.55800000 & -4.05100000 \\
\hline $\mathrm{H}$ & -3.24300000 & -3.91500000 & 4.71800000 & $\mathrm{H}$ & -10.76300000 & -6.49200000 & -3.89000000 \\
\hline $\mathrm{N}$ & -3.20700000 & -4.22300000 & 1.85100000 & $\mathrm{H}$ & -10.83400000 & -4.74000000 & -3.66600000 \\
\hline $\mathrm{H}$ & -2.46200000 & -3.52800000 & 1.81500000 & $\mathrm{C}$ & -8.85200000 & -5.59900000 & -3.32900000 \\
\hline C & -3.56800000 & -5.04500000 & 2.91500000 & $\mathrm{H}$ & -8.85000000 & -4.86200000 & -2.51800000 \\
\hline C & -3.94900000 & -4.57800000 & 0.78100000 & $\mathrm{H}$ & -8.06300000 & -5.25500000 & -3.99200000 \\
\hline $\mathrm{H}$ & -3.86900000 & -4.11200000 & -0.18700000 & C & -8.47700000 & -6.89700000 & -2.60200000 \\
\hline $\mathrm{N}$ & -4.77300000 & -5.57200000 & 1.08000000 & $\mathrm{O}$ & -9.41500000 & -7.54700000 & -2.09100000 \\
\hline C & -4.53600000 & -5.87500000 & 2.40400000 & C & -9.50700000 & -3.82200000 & -5.80400000 \\
\hline $\mathrm{H}$ & -5.05800000 & -6.67700000 & 2.90200000 & $\mathrm{O}$ & -8.31400000 & -3.79800000 & -6.23700000 \\
\hline C & -1.52700000 & -10.15400000 & -0.70100000 & $\mathrm{O}$ & -10.28000000 & -2.88700000 & -5.53300000 \\
\hline $\mathrm{H}$ & -0.94700000 & -11.06600000 & -0.54800000 & $\mathrm{~N}$ & -7.15500000 & -7.18500000 & -2.33800000 \\
\hline $\mathrm{H}$ & -2.09000000 & -10.28600000 & -1.63200000 & $\mathrm{C}$ & -6.24500000 & -7.29800000 & -3.49200000 \\
\hline $\mathrm{N}$ & -0.85300000 & -7.71900000 & -1.15200000 & $\mathrm{H}$ & -5.40900000 & -7.90200000 & -3.12000000 \\
\hline $\mathrm{H}$ & -1.79300000 & -7.33100000 & -1.27700000 & C & -5.62400000 & -5.99300000 & -4.06500000 \\
\hline C & -0.54400000 & -9.02600000 & -0.81600000 & $\mathrm{H}$ & -6.19800000 & -5.12100000 & -3.75500000 \\
\hline C & 0.31100000 & -7.02100000 & -1.19700000 & $\mathrm{H}$ & -5.56100000 & -6.01200000 & -5.15800000 \\
\hline H & 0.34900000 & -5.96200000 & -1.42000000 & $S$ & -3.89500000 & -5.66900000 & -3.46600000 \\
\hline$N$ & 1.35400000 & -7.78500000 & -0.92800000 & $S$ & -3.87300000 & -6.76700000 & -1.69100000 \\
\hline C & 0.82800000 & -9.03600000 & -0.69100000 & C & -6.86100000 & -8.12100000 & -4.62500000 \\
\hline H & 1.44900000 & -9.88200000 & -0.43300000 & $\mathrm{O}$ & -8.09700000 & -8.24500000 & -4.75600000 \\
\hline C & -9.77300000 & -3.30200000 & 2.76700000 & $\mathrm{~N}$ & -6.04500000 & -8.73600000 & -5.49200000 \\
\hline $\mathrm{H}$ & -10.26500000 & -2.50800000 & 2.18900000 & $\mathrm{H}$ & -5.02900000 & -8.63400000 & -5.41500000 \\
\hline $\mathrm{H}$ & -8.93800000 & -2.83200000 & 3.29500000 & C & -6.60700000 & -9.55300000 & -6.56900000 \\
\hline $\mathrm{N}$ & -10.01600000 & -5.28000000 & 1.17600000 & $\mathrm{H}$ & -7.03700000 & -10.47800000 & -6.17200000 \\
\hline $\mathrm{H}$ & -11.04300000 & -5.34400000 & 1.18700000 & $\mathrm{H}$ & -5.77900000 & -9.82500000 & -7.22600000 \\
\hline C & -9.23500000 & -4.37900000 & 1.87400000 & C & -7.69400000 & -8.84900000 & -7.41400000 \\
\hline C & -9.20500000 & -6.07700000 & 0.45000000 & $\mathrm{O}$ & -8.70500000 & -9.54400000 & -7.70500000 \\
\hline $\mathrm{H}$ & -9.56100000 & -6.86300000 & -0.19700000 & $\mathrm{O}$ & -7.47900000 & -7.67000000 & -7.81900000 \\
\hline $\mathrm{N}$ & -7.93700000 & -5.72800000 & 0.61300000 & $\mathrm{Fe}$ & -6.11900000 & -6.43100000 & -0.57400000 \\
\hline C & -7.94500000 & -4.68800000 & 1.51900000 & 0 & -5.51800000 & -8.43700000 & -0.00500000 \\
\hline
\end{tabular}




\begin{tabular}{|c|c|c|c|c|c|c|c|}
\hline 0 & -6.41700000 & -8.00900000 & 0.83700000 & $\mathrm{H}$ & -8.33700000 & -5.56800000 & -6.61800000 \\
\hline & & & & $\mathrm{H}$ & -9.50800000 & -6.35500000 & -7.44900000 \\
\hline 7'TS1 & & & & $\mathrm{H}$ & -8.95100000 & -6.98600000 & -5.98300000 \\
\hline-431 & 12.082142 (a.u.) & & & C & -10.11800000 & -5.17800000 & -5.78400000 \\
\hline $\mathrm{C}$ & -3.11400000 & -4.98300000 & 4.13800000 & $\mathrm{H}$ & -11.10000000 & -5.22800000 & -6.24900000 \\
\hline $\mathrm{H}$ & -3.71100000 & -5.65800000 & 4.75400000 & C & -10.20200000 & -5.55000000 & -4.30100000 \\
\hline $\mathrm{H}$ & -3.33400000 & -3.96700000 & 4.48900000 & $\mathrm{H}$ & -10.68900000 & -6.52100000 & -4.18200000 \\
\hline $\mathrm{N}$ & -3.14300000 & -4.36300000 & 1.63400000 & $\mathrm{H}$ & -10.86800000 & -4.80600000 & -3.85500000 \\
\hline $\mathrm{H}$ & -2.40200000 & -3.66100000 & 1.60600000 & C & -8.81900000 & -5.55300000 & -3.61800000 \\
\hline C & -3.53800000 & -5.15400000 & 2.71000000 & $\mathrm{H}$ & -8.71200000 & -4.67600000 & -2.97100000 \\
\hline C & -3.89000000 & -4.71400000 & 0.56100000 & $\mathrm{H}$ & -8.01500000 & -5.41200000 & -4.33900000 \\
\hline $\mathrm{H}$ & -3.79000000 & -4.26700000 & -0.41600000 & C & -8.54000000 & -6.75400000 & -2.71800000 \\
\hline $\mathrm{N}$ & -4.74400000 & -5.68000000 & 0.87300000 & 0 & -9.51200000 & -7.28200000 & -2.13800000 \\
\hline $\mathrm{C}$ & -4.52100000 & -5.97200000 & 2.20200000 & $\mathrm{C}$ & -9.54200000 & -3.73900000 & -5.96800000 \\
\hline $\mathrm{H}$ & -5.06200000 & -6.75800000 & 2.70400000 & 0 & -8.33900000 & -3.67900000 & -6.36100000 \\
\hline $\mathrm{C}$ & -1.46500000 & -10.23300000 & -0.37100000 & 0 & -10.33800000 & -2.82800000 & -5.67200000 \\
\hline $\mathrm{H}$ & -0.81000000 & -11.10200000 & -0.30400000 & $\mathrm{~N}$ & -7.24300000 & -7.11900000 & -2.43900000 \\
\hline $\mathrm{H}$ & -2.03100000 & -10.32600000 & -1.30500000 & C & -6.32900000 & -7.34400000 & -3.57300000 \\
\hline $\mathrm{N}$ & -1.04400000 & -7.71200000 & -0.53700000 & $\mathrm{H}$ & -5.62500000 & -8.09700000 & -3.21400000 \\
\hline $\mathrm{H}$ & -2.01700000 & -7.44500000 & -0.67600000 & C & -5.46100000 & -6.14000000 & -4.01000000 \\
\hline $\mathrm{C}$ & -0.59800000 & -9.01200000 & -0.36700000 & $\mathrm{H}$ & -5.99600000 & -5.20500000 & -3.84400000 \\
\hline C & 0.02900000 & -6.88500000 & -0.42000000 & $\mathrm{H}$ & -5.23500000 & -6.22700000 & -5.07400000 \\
\hline $\mathrm{H}$ & -0.04200000 & -5.80900000 & -0.50200000 & S & -3.85700000 & -6.02200000 & -3.08700000 \\
\hline $\mathrm{N}$ & 1.14000000 & -7.56700000 & -0.20300000 & $S$ & -4.31900000 & -7.72100000 & -1.34300000 \\
\hline C & 0.75800000 & -8.89100000 & -0.17500000 & C & -6.97600000 & -8.06700000 & -4.75900000 \\
\hline $\mathrm{H}$ & 1.46400000 & -9.69400000 & -0.01400000 & 0 & -8.21600000 & -8.12000000 & -4.93500000 \\
\hline C & -9.79000000 & -3.47500000 & 2.70000000 & $\mathrm{~N}$ & -6.14900000 & -8.70000000 & -5.59600000 \\
\hline $\mathrm{H}$ & -10.30500000 & -2.73300000 & 2.07600000 & $\mathrm{H}$ & -5.13700000 & -8.67900000 & -5.44300000 \\
\hline $\mathrm{H}$ & -8.95400000 & -2.95800000 & 3.17900000 & C & -6.68700000 & -9.45900000 & -6.71500000 \\
\hline $\mathrm{N}$ & -10.01500000 & -5.58500000 & 1.27300000 & $\mathrm{H}$ & -7.32700000 & -10.26700000 & -6.34800000 \\
\hline $\mathrm{H}$ & -11.04200000 & -5.62600000 & 1.26400000 & $\mathrm{H}$ & -5.84700000 & -9.91600000 & -7.24300000 \\
\hline C & -9.24300000 & -4.60800000 & 1.88200000 & C & -7.50200000 & -8.61600000 & -7.72300000 \\
\hline C & -9.19900000 & -6.42900000 & 0.61400000 & 0 & -8.35000000 & -9.27800000 & -8.40700000 \\
\hline $\mathrm{H}$ & -9.54500000 & -7.26600000 & 0.03200000 & 0 & -7.26900000 & -7.39100000 & -7.81900000 \\
\hline $\mathrm{N}$ & -7.93500000 & -6.04600000 & 0.74000000 & $\mathrm{Fe}$ & -6.31000000 & -6.44400000 & -0.67000000 \\
\hline$C$ & -7.95300000 & -4.92300000 & 1.54600000 & $\mathrm{O}$ & -5.14800000 & -8.92300000 & -0.28100000 \\
\hline $\mathrm{H}$ & -7.03900000 & -4.42100000 & 1.81800000 & 0 & -6.29200000 & -8.29600000 & 0.26000000 \\
\hline $\mathrm{C}$ & -7.15900000 & -2.32700000 & -0.60000000 & & & & \\
\hline $\mathrm{H}$ & -6.83100000 & -2.56200000 & 0.41300000 & 'IM1 & $1 a$ & & \\
\hline $\mathrm{H}$ & -8.25300000 & -2.36300000 & -0.61600000 & -431 & 12.171595 (a.u.) & & \\
\hline C & -6.68700000 & -3.40300000 & -1.59700000 & C & -3.04800000 & -5.05300000 & 4.34300000 \\
\hline 0 & -6.76500000 & -3.20000000 & -2.81200000 & $\mathrm{H}$ & -3.62700000 & -5.74000000 & 4.96200000 \\
\hline 0 & -6.31500000 & -4.50200000 & -1.03700000 & $\mathrm{H}$ & -3.24700000 & -4.04800000 & 4.73400000 \\
\hline $\mathrm{N}$ & -9.20700000 & -6.11800000 & -6.49700000 & $\mathrm{~N}$ & -3.18700000 & -4.31100000 & 1.89000000 \\
\hline
\end{tabular}




\begin{tabular}{|c|c|c|c|c|c|c|c|}
\hline $\mathrm{H}$ & -2.44200000 & -3.61300000 & 1.86200000 & C & -8.83800000 & -5.66600000 & -3.50200000 \\
\hline r & -3.52000000 & -5.17700000 & 2.92700000 & $\mathrm{H}$ & -8.73200000 & -4.81000000 & -2.82800000 \\
\hline & -3.92600000 & -4.64700000 & 0.81400000 & $\mathrm{H}$ & -8.06500000 & -5.47600000 & -4.24400000 \\
\hline & -3.87800000 & -4.12400000 & -0.12700000 & C & -8.49200000 & -6.89500000 & -2.66200000 \\
\hline & -4.72300000 & -5.67600000 & 1.08100000 & 0 & -9.40200000 & -7.54000000 & -2.12100000 \\
\hline & -4.46600000 & -6.01700000 & 2.39300000 & C & -9.58200000 & -3.99900000 & -5.95500000 \\
\hline & -4.96300000 & -6.84900000 & 2.86600000 & 0 & -8.38500000 & -3.97600000 & -6.36400000 \\
\hline & -1.37700000 & -10.43400000 & -0.51000000 & 0 & -10.36200000 & -3.06100000 & -5.70100000 \\
\hline & -0.79800000 & -11.31900000 & -0.24700000 & $\mathrm{~N}$ & -7.14500000 & -7.08100000 & -2.37100000 \\
\hline & -1.87100000 & -10.64900000 & -1.46300000 & C & -6.20300000 & -7.37700000 & -3.45100000 \\
\hline & -0.76600000 & -8.16100000 & -1.38400000 & $\mathrm{H}$ & -5.40000000 & -7.96400000 & -2.99700000 \\
\hline & -1.74000000 & -8.03200000 & -1.72500000 & C & -5.59200000 & -6.08600000 & -4.08600000 \\
\hline & -0.42800000 & -9.28800000 & -0.66800000 & $\mathrm{H}$ & -6.16600000 & -5.21200000 & -3.78200000 \\
\hline & 0.29700000 & -7.31800000 & -1.34400000 & $\mathrm{H}$ & -5.58300000 & -6.15200000 & -5.17800000 \\
\hline & 0.28900000 & -6.34000000 & -1.80700000 & $S$ & -3.85500000 & -5.73700000 & -3.56800000 \\
\hline & 1.30900000 & -7.83700000 & -0.66900000 & $S$ & -3.73500000 & -6.62800000 & -1.65200000 \\
\hline & 0.86300000 & -9.07200000 & -0.24400000 & C & -6.81900000 & -8.24000000 & -4.56100000 \\
\hline & 1.48600000 & -9.73700000 & 0.33900000 & 0 & -8.06100000 & -8.30200000 & -4.71200000 \\
\hline & -9.78600000 & -3.34200000 & 2.73200000 & $\mathrm{~N}$ & -5.99900000 & -8.88400000 & -5.38800000 \\
\hline - & -10.30000000 & -2.59400000 & 2.11300000 & $\mathrm{H}$ & -4.98100000 & -8.80300000 & -5.30500000 \\
\hline $\mathrm{H}$ & -8.96300000 & -2.82300000 & 3.23400000 & C & -6.55200000 & -9.58900000 & -6.53700000 \\
\hline $\mathrm{J}$ & -9.94500000 & -5.43200000 & 1.26000000 & $\mathrm{H}$ & -7.19600000 & -10.40800000 & -6.20400000 \\
\hline $\mathrm{H}$ & -10.97100000 & -5.50900000 & 1.24900000 & $\mathrm{H}$ & -5.72100000 & -10.02000000 & -7.09900000 \\
\hline & -9.20800000 & -4.45200000 & 1.90400000 & C & -7.36100000 & -8.66900000 & -7.47700000 \\
\hline$c$ & -9.09100000 & -6.25900000 & 0.62200000 & 0 & -8.27100000 & -9.25400000 & -8.15600000 \\
\hline $\mathrm{H}$ & -9.40000000 & -7.11800000 & 0.05000000 & 0 & -7.07900000 & -7.45400000 & -7.51300000 \\
\hline $\mathrm{N}$ & -7.83900000 & -5.85500000 & 0.78900000 & $\mathrm{Fe}$ & -6.15900000 & -6.44000000 & -0.51700000 \\
\hline & -7.90200000 & -4.74200000 & 1.60200000 & 0 & -3.40000000 & -8.15800000 & -1.87200000 \\
\hline $\mathrm{H}$ & -7.00500000 & -4.22900000 & 1.90800000 & $\mathrm{O}$ & -6.33000000 & -8.22200000 & -0.32100000 \\
\hline & -7.11300000 & -2.33700000 & -0.60400000 & & & & \\
\hline & -6.77200000 & -2.54900000 & 0.40800000 & \multicolumn{2}{|c|}{${ }^{7} \mathrm{TS} 2 \mathrm{a}$} & & \\
\hline & -8.20700000 & -2.37500000 & -0.61300000 & \multicolumn{2}{|c|}{-4312.147392 (a.u.) } & & \\
\hline 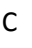 & -6.64400000 & -3.42300000 & -1.59700000 & C & -3.14900000 & -4.90000000 & 4.10500000 \\
\hline 0 & -6.91900000 & -3.28000000 & -2.80100000 & $\mathrm{H}$ & -3.75800000 & -5.56600000 & 4.72100000 \\
\hline & -6.06600000 & -4.43800000 & -1.07100000 & $\mathrm{H}$ & -3.36000000 & -3.88000000 & 4.45100000 \\
\hline$N$ & -9.30200000 & -6.43500000 & -6.34400000 & $\mathrm{~N}$ & -3.18800000 & -4.26900000 & 1.60400000 \\
\hline & -8.46100000 & -5.91600000 & -6.65700000 & $\mathrm{H}$ & -2.44100000 & -3.57300000 & 1.57500000 \\
\hline נr & -9.71800000 & -6.85200000 & -7.18100000 & C & -3.56800000 & -5.07100000 & 2.67500000 \\
\hline$\pi$ & -8.94700000 & -7.20100000 & -5.73600000 & C & -3.93900000 & -4.62300000 & 0.53300000 \\
\hline 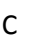 & -10.17200000 & -5.42000000 & -5.66800000 & $\mathrm{H}$ & -3.84300000 & -4.16800000 & -0.44100000 \\
\hline & -11.16600000 & -5.48200000 & -6.10300000 & $\mathrm{~N}$ & -4.77600000 & -5.60400000 & 0.84000000 \\
\hline 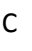 & -10.23400000 & -5.67900000 & -4.15900000 & C & -4.53800000 & -5.90100000 & 2.16500000 \\
\hline & -10.75200000 & -6.61800000 & -3.94700000 & $\mathrm{H}$ & -5.06100000 & -6.70200000 & 2.66400000 \\
\hline & -10.86000000 & -4.87100000 & -3.77000000 & C & -1.32600000 & -10.44600000 & -0.36500000 \\
\hline
\end{tabular}




\begin{tabular}{|c|c|c|c|c|c|c|c|}
\hline $\mathrm{H}$ & -0.68800000 & -11.27600000 & -0.06600000 & $\mathrm{~N}$ & -7.35000000 & -7.14300000 & -2.36000000 \\
\hline $\mathrm{H}$ & -1.81700000 & -10.74200000 & -1.29500000 & C & -6.36700000 & -7.42400000 & -3.40500000 \\
\hline & -0.80700000 & -8.26100000 & -1.51000000 & $\mathrm{H}$ & -5.72000000 & -8.20400000 & -2.99200000 \\
\hline & -1.70900000 & -8.21600000 & -1.96600000 & C & -5.45000000 & -6.24100000 & -3.84800000 \\
\hline & -0.45600000 & -9.26100000 & -0.62200000 & $\mathrm{H}$ & -5.95500000 & -5.29000000 & -3.67900000 \\
\hline & 0.20500000 & -7.34800000 & -1.53900000 & $\mathrm{H}$ & -5.20800000 & -6.33700000 & -4.90800000 \\
\hline & 0.16800000 & -6.44700000 & -2.13600000 & $S$ & -3.83300000 & -6.08700000 & -2.94900000 \\
\hline & 1.19300000 & -7.71000000 & -0.74700000 & $S$ & -4.06000000 & -7.65600000 & -1.27500000 \\
\hline & 0.79300000 & -8.90100000 & -0.17700000 & $\mathrm{C}$ & -6.98400000 & -8.11200000 & -4.64600000 \\
\hline & 1.42000000 & -9.44200000 & 0.51800000 & 0 & -8.22000000 & -8.14400000 & -4.84400000 \\
\hline & -9.87700000 & -3.49400000 & 2.74800000 & $\mathrm{~N}$ & -6.15400000 & -8.71000000 & -5.50600000 \\
\hline & -10.41800000 & -2.78300000 & 2.11200000 & $\mathrm{H}$ & -5.14000000 & -8.68400000 & -5.38500000 \\
\hline & -9.03600000 & -2.95100000 & 3.19000000 & $\mathrm{C}$ & -6.68800000 & -9.45300000 & -6.64100000 \\
\hline & -10.11700000 & -5.64000000 & 1.37300000 & $\mathrm{H}$ & -7.34200000 & -10.25400000 & -6.28400000 \\
\hline & -11.14400000 & -5.64400000 & 1.31500000 & $\mathrm{H}$ & -5.84400000 & -9.91700000 & -7.15600000 \\
\hline & -9.33800000 & -4.65800000 & 1.96900000 & $\mathrm{C}$ & -7.47300000 & -8.59600000 & -7.66000000 \\
\hline & -9.30800000 & -6.54500000 & 0.79900000 & 0 & -8.33600000 & -9.23700000 & -8.34600000 \\
\hline & -9.64500000 & -7.38700000 & 0.21600000 & 0 & -7.20400000 & -7.37900000 & -7.76100000 \\
\hline & -8.03800000 & -6.20000000 & 0.97600000 & $\mathrm{Fe}$ & -6.54700000 & -6.56200000 & -0.45200000 \\
\hline & -8.04700000 & -5.03200000 & 1.71700000 & 0 & -3.76400000 & -9.01500000 & -1.93300000 \\
\hline & -7.12800000 & -4.54400000 & 1.99800000 & 0 & -5.77300000 & -8.15700000 & -0.09600000 \\
\hline & -7.16000000 & -2.36800000 & -0.58100000 & & & & \\
\hline & -6.87300000 & -2.58800000 & 0.44900000 & \multicolumn{2}{|c|}{${ }^{7} \mathrm{IM} 2 \mathrm{a}$} & & \\
\hline & -8.25400000 & -2.41800000 & -0.62800000 & \multicolumn{2}{|c|}{-4312.199066 (a.u.) } & & \\
\hline $\mathcal{L}$ & -6.64700000 & -3.47600000 & -1.52500000 & C & -3.40200000 & -4.73800000 & 3.15300000 \\
\hline ? & -6.40900000 & -3.24400000 & -2.71500000 & $\mathrm{H}$ & -4.13400000 & -5.42900000 & 3.58200000 \\
\hline 0 & -6.60100000 & -4.62900000 & -0.96000000 & $\mathrm{H}$ & -3.65800000 & -3.73700000 & 3.52100000 \\
\hline & -9.19100000 & -6.14300000 & -6.49900000 & $\mathrm{~N}$ & -2.88800000 & -3.95400000 & 0.74500000 \\
\hline+ & -8.30700000 & -5.62300000 & -6.63500000 & $\mathrm{H}$ & -2.13900000 & -3.27600000 & 0.92400000 \\
\hline & -9.49600000 & -6.40000000 & -7.44400000 & C & -3.52900000 & -4.78700000 & 1.65500000 \\
\hline & -8.95600000 & -7.00200000 & -5.96200000 & C & -3.38200000 & -4.21600000 & -0.48500000 \\
\hline & -10.09200000 & -5.16900000 & -5.81300000 & $\mathrm{H}$ & -3.06200000 & -3.70100000 & -1.37900000 \\
\hline $\mathrm{H}$ & -11.07500000 & -5.22500000 & -6.27400000 & $\mathrm{~N}$ & -4.30900000 & -5.16400000 & -0.43200000 \\
\hline C & -10.16400000 & -5.49700000 & -4.32300000 & C & -4.40500000 & -5.52800000 & 0.89800000 \\
\hline $\mathrm{H}$ & -10.65800000 & -6.46100000 & -4.17200000 & $\mathrm{H}$ & -5.11300000 & -6.27900000 & 1.21400000 \\
\hline $\mathrm{H}$ & -10.81500000 & -4.73800000 & -3.88100000 & C & -1.53300000 & -10.35100000 & -0.68500000 \\
\hline 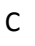 & -8.77100000 & -5.50400000 & -3.66100000 & $\mathrm{H}$ & -0.72400000 & -11.04100000 & -0.43300000 \\
\hline H & -8.61000000 & -4.57800000 & -3.09900000 & $\mathrm{H}$ & -2.02200000 & -10.74600000 & -1.58100000 \\
\hline $\mathrm{H}$ & -7.97100000 & -5.48700000 & -4.40200000 & $\mathrm{~N}$ & -1.67300000 & -7.93400000 & -1.47400000 \\
\hline 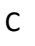 & -8.57900000 & -6.63500000 & -2.65900000 & $\mathrm{H}$ & -2.66400000 & -7.95400000 & -1.70000000 \\
\hline & -9.58700000 & -6.99400000 & -2.01000000 & C & -0.95000000 & -9.00000000 & -0.96000000 \\
\hline 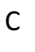 & -9.50400000 & -3.74200000 & -6.02200000 & C & -0.82200000 & -6.87600000 & -1.58200000 \\
\hline & -8.29500000 & -3.69500000 & -6.39800000 & $\mathrm{H}$ & -1.14000000 & -5.91400000 & -1.95700000 \\
\hline & -10.29700000 & -2.82200000 & -5.74400000 & $\mathrm{~N}$ & 0.39600000 & -7.18800000 & -1.18600000 \\
\hline
\end{tabular}




\begin{tabular}{|c|c|c|c|c|c|c|c|}
\hline & 0.32500000 & -8.51100000 & -0.80300000 & C & -7.07600000 & -8.40700000 & -5.25000000 \\
\hline 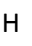 & 1.18900000 & -9.04300000 & -0.42700000 & 0 & -8.31500000 & -8.39100000 & -5.25800000 \\
\hline & -9.80400000 & -3.18400000 & 2.78400000 & $\mathrm{~N}$ & -6.39300000 & -9.11000000 & -6.16400000 \\
\hline & -10.32800000 & -2.40100000 & 2.22200000 & $\mathrm{H}$ & -5.37600000 & -9.13500000 & -6.16900000 \\
\hline & -8.96600000 & -2.70100000 & 3.29600000 & C & -7.14100000 & -9.90400000 & -7.12100000 \\
\hline & -10.06900000 & -5.10900000 & 1.14200000 & $\mathrm{H}$ & -7.61100000 & -10.75600000 & -6.62000000 \\
\hline & -11.09600000 & -5.12600000 & 1.10300000 & $\mathrm{H}$ & -6.43200000 & -10.30600000 & -7.84900000 \\
\hline & -9.27400000 & -4.27300000 & 1.90100000 & C & -8.24600000 & -9.15800000 & -7.89800000 \\
\hline & -9.26400000 & -6.04900000 & 0.58800000 & $\mathrm{O}$ & -9.11200000 & -9.93100000 & -8.40200000 \\
\hline & -9.65200000 & -6.85100000 & -0.02400000 & 0 & -8.20100000 & -7.90600000 & -8.04600000 \\
\hline & -7.99700000 & -5.86800000 & 0.91000000 & $\mathrm{Fe}$ & -5.75000000 & -5.82800000 & -2.01600000 \\
\hline & -7.99700000 & -4.76000000 & 1.72500000 & 0 & -4.41800000 & -9.33200000 & -1.89500000 \\
\hline & -7.07600000 & -4.37500000 & 2.14200000 & $\mathrm{O}$ & -5.93700000 & -7.48600000 & -0.90800000 \\
\hline & -7.19000000 & -2.01800000 & -1.09800000 & & & & \\
\hline & -7.21900000 & -2.42700000 & -0.08500000 & & & & \\
\hline & -8.21900000 & -1.98600000 & -1.46800000 & -40 & 46.231069 (a.u.) & & \\
\hline & -6.45400000 & -2.99500000 & -2.01400000 & $\mathrm{~N}$ & -4.79000000 & -0.48200000 & 3.66000000 \\
\hline & -5.74600000 & -2.66500000 & -2.96500000 & $\mathrm{H}$ & -4.63100000 & -1.34300000 & 3.17400000 \\
\hline & -6.75000000 & -4.22700000 & -1.69000000 & $\mathrm{C}$ & -6.17200000 & -0.09400000 & 3.81600000 \\
\hline & -8.47700000 & -5.70800000 & -5.91900000 & $\mathrm{H}$ & -6.36900000 & 0.11600000 & 4.86200000 \\
\hline & -7.87000000 & -5.86700000 & -5.07900000 & C & -6.53300000 & 1.12400000 & 2.92200000 \\
\hline & -8.07500000 & -4.91000000 & -6.43700000 & $\mathrm{H}$ & -7.54800000 & 1.45600000 & 3.14300000 \\
\hline & -8.47400000 & -6.57200000 & -6.49200000 & $\mathrm{H}$ & -5.86000000 & 1.91600000 & 3.26700000 \\
\hline & -9.84000000 & -5.24700000 & -5.49700000 & $\mathrm{~N}$ & -5.25900000 & 0.60800000 & 0.73500000 \\
\hline & -10.53400000 & -5.56300000 & -6.28200000 & $\mathrm{H}$ & -4.33600000 & 0.35200000 & 1.10200000 \\
\hline & -10.30600000 & -5.86800000 & -4.17400000 & C & -6.41300000 & 0.92500000 & 1.44500000 \\
\hline & -10.59300000 & -6.90900000 & -4.34000000 & $\mathrm{C}$ & -5.55100000 & 0.61900000 & -0.57800000 \\
\hline & -11.22500000 & -5.32000000 & -3.94500000 & $\mathrm{H}$ & -4.84200000 & 0.41000000 & -1.36200000 \\
\hline & -9.33900000 & -5.79600000 & -2.96100000 & $\mathrm{~N}$ & -6.83100000 & 0.92600000 & -0.76100000 \\
\hline & -9.93700000 & -5.87100000 & -2.04900000 & C & -7.38000000 & 1.11000000 & 0.49200000 \\
\hline & -8.81400000 & -4.83700000 & -2.92900000 & $\mathrm{H}$ & -8.42500000 & 1.33600000 & 0.63600000 \\
\hline & -8.32400000 & -6.95700000 & -2.94300000 & C & -7.01700000 & -1.28400000 & 3.42200000 \\
\hline & -8.66000000 & -8.05400000 & -2.47000000 & $\mathrm{O}$ & -6.46800000 & -2.34300000 & 3.11700000 \\
\hline & -9.85300000 & -3.67900000 & -5.52900000 & $\mathrm{~N}$ & -9.35900000 & -3.42500000 & 0.77300000 \\
\hline & -8.87200000 & -3.12100000 & -6.09700000 & $\mathrm{H}$ & -9.32700000 & -4.26100000 & 1.31300000 \\
\hline & -10.85700000 & -3.12800000 & -5.03500000 & $\mathrm{C}$ & -9.48400000 & -3.58600000 & -0.66200000 \\
\hline & -7.11000000 & -6.61900000 & -3.50300000 & $\mathrm{H}$ & -9.14300000 & -2.69000000 & -1.16300000 \\
\hline & -6.27600000 & -7.60900000 & -4.20800000 & $\mathrm{C}$ & -8.66000000 & -4.79300000 & -1.16500000 \\
\hline & -5.81400000 & -8.35800000 & -3.55300000 & $\mathrm{H}$ & -9.05300000 & -5.71900000 & -0.69200000 \\
\hline & -5.16500000 & -6.77900000 & -4.86700000 & $\mathrm{H}$ & -8.77400000 & -4.90400000 & -2.26400000 \\
\hline & -5.60000000 & -5.97700000 & -5.46400000 & $\mathrm{~N}$ & -6.35100000 & -3.69600000 & -1.15700000 \\
\hline & -4.53100000 & -7.36500000 & & $\mathrm{H}$ & -6.62200000 & & \\
\hline & -4.08100000 & -5.99900000 & -3.58900000 & C & -7.18700000 & -4.75400000 & -0.87300000 \\
\hline & -5.63300000 & -9.02000000 & -1.00400000 & C & -5.09000000 & -4.09100000 & -0.86600000 \\
\hline
\end{tabular}




\begin{tabular}{|c|c|c|c|c|c|c|c|}
\hline H & -4.21500000 & -3.44700000 & -0.98800000 & C & -7.78900000 & 3.18700000 & -6.99900000 \\
\hline $\mathrm{N}$ & -5.05800000 & -5.33100000 & -0.42200000 & $\mathrm{H}$ & -8.38000000 & 3.93300000 & -6.45800000 \\
\hline C & -6.37900000 & -5.74800000 & -0.41600000 & $\mathrm{H}$ & -6.74300000 & 3.30200000 & -6.70300000 \\
\hline $\mathrm{H}$ & -6.63800000 & -6.75200000 & -0.10200000 & C & -8.35100000 & 1.83200000 & -6.59400000 \\
\hline C & -10.93100000 & -3.82000000 & -1.07100000 & $\mathrm{O}$ & -9.57600000 & 1.65100000 & -6.54800000 \\
\hline O & -11.80100000 & -4.09200000 & -0.24400000 & C & -5.49000000 & 2.97200000 & -9.07400000 \\
\hline $\mathrm{N}$ & -8.87800000 & 8.43600000 & -0.17400000 & $\mathrm{O}$ & -4.82600000 & 1.94800000 & -8.73200000 \\
\hline $\mathrm{H}$ & -8.17600000 & 8.26700000 & 0.51200000 & 0 & -5.15100000 & 4.16800000 & -9.14200000 \\
\hline C & -9.24200000 & 7.34900000 & -1.06900000 & $\mathrm{~N}$ & -7.39900000 & 0.88900000 & -6.41100000 \\
\hline $\mathrm{H}$ & -9.71100000 & 7.74500000 & -1.96700000 & $\mathrm{H}$ & -6.46800000 & 1.24900000 & -6.17700000 \\
\hline C & -8.01500000 & 6.48900000 & -1.47800000 & C & -7.59700000 & -0.54800000 & -6.2410000 \\
\hline $\mathrm{H}$ & -7.45100000 & 7.10900000 & -2.18600000 & $\mathrm{H}$ & -8.25700000 & -0.74400000 & -5.38500000 \\
\hline H & -7.38900000 & 6.36900000 & -0.58900000 & C & -6.22100000 & -1.19000000 & -6.03100000 \\
\hline $\mathrm{N}$ & -9.04300000 & 4.86000000 & -3.17100000 & $\mathrm{H}$ & -5.47800000 & -0.69300000 & -6.66200000 \\
\hline H & -9.57500000 & 5.54900000 & -3.72400000 & $\mathrm{H}$ & -6.23400000 & -2.23000000 & -6.33300000 \\
\hline C & -8.29100000 & 5.12800000 & -2.03800000 & $\mathrm{~S}$ & -5.47600000 & -1.14900000 & -4.33300000 \\
\hline C & -9.03700000 & 3.53100000 & -3.38800000 & $\mathrm{~S}$ & -7.05200000 & -1.25200000 & -3.02000000 \\
\hline $\mathrm{H}$ & -9.56500000 & 3.04800000 & -4.19400000 & C & -8.28400000 & -1.13600000 & -7.4970000 \\
\hline N & -8.30600000 & 2.92100000 & -2.45300000 & $\mathrm{O}$ & -8.89500000 & -0.39200000 & -8.28700000 \\
\hline C & -7.85200000 & 3.90600000 & -1.60300000 & $\mathrm{~N}$ & -8.20900000 & -2.44400000 & -7.75400000 \\
\hline $\mathrm{H}$ & -7.24200000 & 3.66100000 & -0.75200000 & $\mathrm{H}$ & -7.73600000 & -3.09300000 & -7.13000000 \\
\hline C & -10.28600000 & 6.48400000 & -0.39200000 & C & -8.63800000 & -2.87800000 & $-9.0790000 c$ \\
\hline O & -11.44900000 & 6.50300000 & -0.77300000 & $\mathrm{H}$ & -9.72400000 & -2.79800000 & $-9.1770000 c$ \\
\hline$N$ & -2.84400000 & 3.26500000 & -2.15100000 & $\mathrm{H}$ & -8.36800000 & -3.92900000 & -9.19600000 \\
\hline H & -3.32200000 & 3.24200000 & -1.26200000 & C & -7.98100000 & -2.04500000 & -10.22000000 \\
\hline C & -3.50400000 & 4.01700000 & -3.20400000 & 0 & -8.64800000 & -2.02500000 & -11.30200000 \\
\hline H & -3.06700000 & 3.78000000 & -4.16900000 & 0 & -6.87900000 & -1.48800000 & -10.01700000 \\
\hline C & -5.04300000 & 3.75600000 & -3.23200000 & $\mathrm{Fe}$ & -7.75600000 & 1.00100000 & -2.50300000 \\
\hline $\mathrm{H}$ & -5.38400000 & 3.75000000 & -2.19900000 & $\mathrm{O}$ & -9.39900000 & 0.67600000 & -3.57300000 \\
\hline $\mathrm{H}$ & -5.54600000 & 4.57000000 & -3.76400000 & $\mathrm{O}$ & -9.29500000 & 0.30100000 & -2.2800000 \\
\hline C & -5.45600000 & 2.47200000 & -3.96700000 & & & & \\
\hline O & -5.21900000 & 2.37500000 & -5.18400000 & $\mathbf{R}^{\prime}$ & & & \\
\hline $\mathrm{O}$ & -6.06500000 & 1.57900000 & -3.27000000 & & 046.265623 (a.u.) & & \\
\hline C & -3.34100000 & 5.52000000 & -3.01000000 & $N$ & -4.78000000 & -0.46300000 & 3.62100000 \\
\hline O & -3.67800000 & 6.30300000 & -3.89500000 & $\mathrm{H}$ & -4.62800000 & -1.32800000 & 3.14000000 \\
\hline$N$ & -7.16100000 & 1.22500000 & -9.52700000 & C & -6.15700000 & -0.05400000 & 3.75800000 \\
\hline $\mathrm{H}$ & -6.30200000 & 0.87100000 & -9.06200000 & $\mathrm{H}$ & -6.36000000 & 0.17900000 & 4.79800000 \\
\hline H & -7.12300000 & 0.85200000 & -10.48300000 & C & -6.49300000 & 1.15200000 & 2.83400000 \\
\hline $\mathrm{H}$ & -7.97800000 & 0.78700000 & -9.04700000 & $\mathrm{H}$ & -7.50000000 & 1.50800000 & 3.05100000 \\
\hline C & -6.98300000 & 2.70500000 & -9.46000000 & $\mathrm{H}$ & -5.80100000 & 1.93700000 & 3.16000000 \\
\hline $\mathrm{H}$ & -7.14400000 & 3.09600000 & -10.46400000 & $\mathrm{~N}$ & -5.25700000 & 0.50600000 & 0.65400000 \\
\hline C & -7.95700000 & 3.42700000 & -8.51700000 & $\mathrm{H}$ & -4.33500000 & 0.24800000 & 1.02400000 \\
\hline $\mathrm{H}$ & -8.98900000 & 3.21200000 & -8.81300000 & C & -6.38300000 & 0.92000000 & 1.35900000 \\
\hline H & -7.78800000 & 4.48700000 & -8.72700000 & C & -5.55600000 & 0.48800000 & -0.656000 \\
\hline
\end{tabular}




\begin{tabular}{|c|c|c|c|c|c|c|c|}
\hline $\mathrm{H}$ & -4.86800000 & 0.20000000 & -1.43300000 & C & -5.07100000 & 3.60200000 & -3.10000000 \\
\hline $\mathrm{N}$ & -6.81600000 & 0.87500000 & -0.84300000 & $\mathrm{H}$ & -5.34400000 & 3.38500000 & -2.06900000 \\
\hline C & -7.34200000 & 1.14100000 & 0.40600000 & $\mathrm{H}$ & -5.65200000 & 4.47300000 & -3.42600000 \\
\hline $\mathrm{H}$ & -8.37000000 & 1.43400000 & 0.54800000 & C & -5.49600000 & 2.47800000 & -4.05100000 \\
\hline C & -7.00900000 & -1.24400000 & 3.37900000 & 0 & -5.15000000 & 2.51600000 & -5.24400000 \\
\hline $\mathrm{O}$ & -6.46600000 & -2.30600000 & 3.07800000 & 0 & -6.25800000 & 1.54200000 & -3.59600000 \\
\hline $\mathrm{N}$ & -9.34000000 & -3.40000000 & 0.75100000 & C & -3.40900000 & 5.44100000 & -2.92300000 \\
\hline $\mathrm{H}$ & -9.31100000 & -4.22900000 & 1.30100000 & 0 & -3.79400000 & 6.21700000 & -3.79400000 \\
\hline C & -9.46600000 & -3.57900000 & -0.68100000 & $\mathrm{~N}$ & -7.16200000 & 1.20600000 & -9.54000000 \\
\hline $\mathrm{H}$ & -9.11500000 & -2.69400000 & -1.19400000 & $\mathrm{H}$ & -6.29000000 & 0.86000000 & -9.09400000 \\
\hline C & -8.65600000 & -4.80300000 & -1.16900000 & $\mathrm{H}$ & -7.13300000 & 0.84800000 & -10.50300000 \\
\hline $\mathrm{H}$ & -9.05300000 & -5.71900000 & -0.67900000 & $\mathrm{H}$ & -7.96400000 & 0.74700000 & -9.05200000 \\
\hline $\mathrm{H}$ & -8.77600000 & -4.92900000 & -2.26600000 & C & -7.00700000 & 2.68700000 & -9.45100000 \\
\hline $\mathrm{N}$ & -6.34500000 & -3.72000000 & -1.21400000 & $\mathrm{H}$ & -7.17700000 & 3.09000000 & -10.44900000 \\
\hline $\mathrm{H}$ & -6.62200000 & -2.83100000 & -1.60000000 & C & -7.99200000 & 3.38100000 & -8.49700000 \\
\hline C & -7.18000000 & -4.76600000 & -0.89000000 & $\mathrm{H}$ & -9.02000000 & 3.14700000 & -8.79400000 \\
\hline C & -5.08200000 & -4.10900000 & -0.92400000 & $\mathrm{H}$ & -7.84700000 & 4.44600000 & -8.70000000 \\
\hline $\mathrm{H}$ & -4.20700000 & -3.47200000 & -1.07700000 & C & -7.81400000 & 3.13800000 & -6.98000000 \\
\hline $\mathrm{N}$ & -5.04900000 & -5.33500000 & -0.44100000 & $\mathrm{H}$ & -8.43200000 & 3.85900000 & -6.43300000 \\
\hline C & -6.37100000 & -5.74800000 & -0.41100000 & $\mathrm{H}$ & -6.77100000 & 3.29100000 & -6.68700000 \\
\hline $\mathrm{H}$ & -6.63000000 & -6.74200000 & -0.06400000 & C & -8.31400000 & 1.76200000 & -6.56500000 \\
\hline C & -10.91500000 & -3.80200000 & -1.08700000 & $\mathrm{O}$ & -9.52700000 & 1.53400000 & -6.44700000 \\
\hline $\mathrm{O}$ & -11.78800000 & -4.05000000 & -0.25500000 & C & -5.51500000 & 2.97100000 & -9.07300000 \\
\hline $\mathrm{N}$ & -8.89000000 & 8.43500000 & -0.19000000 & $\mathrm{O}$ & -4.83300000 & 1.95000000 & -8.75700000 \\
\hline $\mathrm{H}$ & -8.18300000 & 8.25900000 & 0.48800000 & 0 & -5.19400000 & 4.17200000 & -9.12600000 \\
\hline C & -9.27100000 & 7.34900000 & -1.08000000 & $\mathrm{~N}$ & -7.33500000 & 0.83900000 & -6.44900000 \\
\hline $\mathrm{H}$ & -9.75300000 & 7.74600000 & -1.97100000 & $\mathrm{H}$ & -6.40000000 & 1.21100000 & -6.26500000 \\
\hline C & -8.05500000 & 6.48200000 & -1.50900000 & C & -7.52600000 & -0.59700000 & -6.26600000 \\
\hline $\mathrm{H}$ & -7.50600000 & 7.09400000 & -2.23600000 & $\mathrm{H}$ & -8.19200000 & -0.78800000 & -5.41300000 \\
\hline $\mathrm{H}$ & -7.41100000 & 6.37000000 & -0.63300000 & C & -6.15800000 & -1.24700000 & -6.03800000 \\
\hline $\mathrm{N}$ & -9.13900000 & 4.83500000 & -3.14700000 & $\mathrm{H}$ & -5.39300000 & -0.71700000 & -6.61100000 \\
\hline $\mathrm{H}$ & -9.67400000 & 5.52300000 & -3.70000000 & $\mathrm{H}$ & -6.16100000 & -2.26700000 & -6.40200000 \\
\hline C & -8.35100000 & 5.11600000 & -2.04700000 & $S$ & -5.47800000 & -1.31600000 & -4.30600000 \\
\hline C & -9.15500000 & 3.50000000 & -3.33600000 & $S$ & -7.10700000 & -1.28100000 & -3.02200000 \\
\hline $\mathrm{H}$ & -9.71500000 & 3.01500000 & -4.12000000 & C & -8.22700000 & -1.18000000 & -7.51700000 \\
\hline $\mathrm{N}$ & -8.40000000 & 2.89600000 & -2.42000000 & $\mathrm{O}$ & -8.85900000 & -0.43100000 & -8.28800000 \\
\hline C & -7.91300000 & 3.89400000 & -1.60400000 & $\mathrm{~N}$ & -8.15400000 & -2.48400000 & -7.78900000 \\
\hline $\mathrm{H}$ & -7.27700000 & 3.66400000 & -0.76800000 & $\mathrm{H}$ & -7.67300000 & -3.14000000 & -7.17600000 \\
\hline C & -10.30800000 & 6.48800000 & -0.38700000 & C & -8.61100000 & -2.90400000 & -9.10800000 \\
\hline 0 & -11.47700000 & 6.51100000 & -0.74800000 & $\mathrm{H}$ & -9.69800000 & -2.81600000 & -9.18400000 \\
\hline $\mathrm{N}$ & -2.82500000 & 3.19800000 & -2.08800000 & $\mathrm{H}$ & -8.35100000 & -3.95500000 & -9.23900000 \\
\hline $\mathrm{H}$ & -3.27900000 & 3.15400000 & -1.18700000 & C & -7.97300000 & -2.06300000 & -10.25400000 \\
\hline C & -3.54000000 & 3.93400000 & -3.11500000 & $\mathrm{O}$ & -8.65500000 & -2.03900000 & -11.32700000 \\
\hline $\mathrm{H}$ & -3.11900000 & 3.72100000 & -4.09300000 & $\mathrm{O}$ & -6.86900000 & -1.50500000 & -10.06400000 \\
\hline
\end{tabular}




\begin{tabular}{|c|c|c|c|c|c|c|c|}
\hline $\mathrm{F}$ & -7.73000000 & 0.96500000 & -2.57800000 & C & -8.07000000 & 6.45300000 & -1.53800000 \\
\hline $\mathrm{c}$ & -9.37900000 & 0.57800000 & -3.53400000 & $\mathrm{H}$ & -7.51800000 & 7.07100000 & -2.25800000 \\
\hline \multirow[t]{2}{*}{$\mathrm{O}$} & -9.45200000 & 0.31800000 & -2.24700000 & $\mathrm{H}$ & -7.42400000 & 6.32200000 & -0.66600000 \\
\hline & & & & $\mathrm{N}$ & -9.19100000 & 4.83800000 & -3.18600000 \\
\hline \multicolumn{2}{|c|}{${ }^{5} \mathbf{R}^{\prime}$} & & & $\mathrm{H}$ & -9.71600000 & 5.53900000 & -3.73000000 \\
\hline \multicolumn{2}{|c|}{-4046.274136 (a.u.) } & & & C & -8.38300000 & 5.09700000 & -2.09300000 \\
\hline $\mathrm{N}$ & -4.78400000 & -0.52300000 & 3.75300000 & $\mathrm{C}$ & -9.23800000 & 3.50400000 & -3.38000000 \\
\hline $\mathrm{H}$ & -4.62200000 & -1.39800000 & 3.29400000 & $\mathrm{H}$ & -9.82300000 & 3.03200000 & -4.15500000 \\
\hline C & -6.16900000 & -0.14500000 & 3.90700000 & $\mathrm{~N}$ & -8.48300000 & 2.88000000 & -2.47900000 \\
\hline $\mathrm{H}$ & -6.36900000 & 0.06300000 & 4.95100000 & C & -7.96100000 & 3.86500000 & -1.66600000 \\
\hline C & -6.53700000 & 1.06800000 & 3.00900000 & $\mathrm{H}$ & -7.32200000 & 3.61700000 & -0.83500000 \\
\hline $\mathrm{H}$ & -7.55200000 & 1.39900000 & 3.22800000 & C & -10.31500000 & 6.46600000 & -0.39400000 \\
\hline $\mathrm{H}$ & -5.86500000 & 1.86600000 & 3.34300000 & 0 & -11.48400000 & 6.49200000 & -0.75300000 \\
\hline $\mathrm{N}$ & -5.27000000 & 0.49300000 & 0.83300000 & $\mathrm{~N}$ & -2.81400000 & 3.29500000 & -2.14600000 \\
\hline $\mathrm{H}$ & -4.34200000 & 0.25400000 & 1.20000000 & $\mathrm{H}$ & -3.30000000 & 3.25400000 & -1.26200000 \\
\hline C & -6.42100000 & 0.84500000 & 1.53600000 & C & -3.46400000 & 4.06700000 & -3.19100000 \\
\hline C & -5.56900000 & 0.45200000 & -0.47400000 & $\mathrm{H}$ & -3.02300000 & 3.84100000 & -4.15600000 \\
\hline $\mathrm{H}$ & -4.86000000 & 0.20800000 & -1.24800000 & $\mathrm{C}$ & -5.00200000 & 3.81300000 & -3.22700000 \\
\hline $\mathrm{N}$ & -6.85200000 & 0.75800000 & -0.66500000 & $\mathrm{H}$ & -5.34900000 & 3.77500000 & -2.19600000 \\
\hline C & -7.39200000 & 0.99700000 & 0.58200000 & $\mathrm{H}$ & -5.50500000 & 4.64100000 & -3.73600000 \\
\hline $\mathrm{H}$ & -8.43500000 & 1.23200000 & 0.72000000 & $\mathrm{C}$ & -5.41100000 & 2.55600000 & -4.00100000 \\
\hline C & -6.99900000 & -1.34700000 & 3.51000000 & 0 & -5.18900000 & 2.48200000 & -5.21700000 \\
\hline 0 & -6.43600000 & -2.40700000 & 3.24000000 & 0 & -6.04500000 & 1.65400000 & -3.32500000 \\
\hline $\mathrm{N}$ & -9.27100000 & -3.47600000 & 0.78100000 & $\mathrm{C}$ & -3.29800000 & 5.56600000 & -2.98100000 \\
\hline $\mathrm{H}$ & -9.19800000 & -4.32100000 & 1.30300000 & $\mathrm{O}$ & -3.64200000 & 6.35900000 & -3.85500000 \\
\hline C & -9.40700000 & -3.61400000 & -0.65700000 & $\mathrm{~N}$ & -7.17500000 & 1.22200000 & -9.59700000 \\
\hline $\mathrm{H}$ & -9.06200000 & -2.71200000 & -1.14400000 & $\mathrm{H}$ & -6.30600000 & 0.87000000 & -9.14900000 \\
\hline C & -8.59300000 & -4.82000000 & -1.18200000 & $\mathrm{H}$ & -7.13700000 & 0.88200000 & -10.56600000 \\
\hline $\mathrm{H}$ & -8.98600000 & -5.74900000 & -0.71300000 & $\mathrm{H}$ & -7.98200000 & 0.75500000 & -9.12200000 \\
\hline $\mathrm{H}$ & -8.71700000 & -4.92100000 & -2.28100000 & C & -7.02000000 & 2.70100000 & -9.48200000 \\
\hline $\mathrm{N}$ & -6.26900000 & -3.77000000 & -1.28900000 & $\mathrm{H}$ & -7.18900000 & 3.12100000 & -10.47300000 \\
\hline $\mathrm{H}$ & -6.53000000 & -2.90900000 & -1.74100000 & C & -8.00400000 & 3.37800000 & -8.51500000 \\
\hline C & -7.11700000 & -4.78400000 & -0.90300000 & $\mathrm{H}$ & -9.03200000 & 3.14200000 & -8.80700000 \\
\hline C & -5.01300000 & -4.14800000 & -0.95400000 & $\mathrm{H}$ & -7.86500000 & 4.44600000 & -8.70700000 \\
\hline $\mathrm{H}$ & -4.13000000 & -3.53200000 & -1.13900000 & C & -7.81400000 & 3.12000000 & -7.00000000 \\
\hline $\mathrm{N}$ & -4.99700000 & -5.33700000 & -0.38600000 & $\mathrm{H}$ & -8.42600000 & 3.83900000 & -6.44300000 \\
\hline C & -6.32200000 & -5.73800000 & -0.34600000 & $\mathrm{H}$ & -6.77000000 & 3.27000000 & -6.71200000 \\
\hline $\mathrm{H}$ & -6.59400000 & -6.70100000 & 0.06900000 & $\mathrm{C}$ & -8.31700000 & 1.74100000 & -6.59700000 \\
\hline C & -10.86000000 & -3.83300000 & -1.06000000 & $\mathrm{O}$ & -9.52900000 & 1.51500000 & -6.47700000 \\
\hline $\mathrm{O}$ & -11.72900000 & -4.08300000 & -0.22600000 & $\mathrm{C}$ & -5.53000000 & 2.97800000 & -9.09500000 \\
\hline $\mathrm{N}$ & -8.88800000 & 8.40800000 & -0.20700000 & 0 & -4.85400000 & 1.95400000 & -8.77700000 \\
\hline $\mathrm{H}$ & -8.17800000 & 8.23100000 & 0.46900000 & 0 & -5.20300000 & 4.17800000 & -9.13900000 \\
\hline C & -9.27900000 & 7.32300000 & -1.09400000 & $\mathrm{~N}$ & -7.34000000 & 0.80900000 & -6.49700000 \\
\hline $\mathrm{H}$ & -9.76600000 & 7.72400000 & -1.98000000 & $\mathrm{H}$ & -6.39900000 & 1.16800000 & -6.32500000 \\
\hline
\end{tabular}




\begin{tabular}{|c|c|c|c|c|c|c|c|}
\hline C & -7.52900000 & -0.63300000 & -6.37500000 & C & -8.60600000 & -4.81200000 & -1.18500000 \\
\hline $\mathrm{H}$ & -8.16000000 & -0.86200000 & -5.50500000 & $\mathrm{H}$ & -8.99400000 & -5.74200000 & -0.71500000 \\
\hline C & -6.15600000 & -1.29200000 & -6.22100000 & $\mathrm{H}$ & -8.73300000 & -4.91300000 & -2.28400000 \\
\hline $\mathrm{H}$ & -5.42600000 & -0.80400000 & -6.87400000 & $\mathrm{~N}$ & -6.28800000 & -3.75100000 & -1.29700000 \\
\hline $\mathrm{H}$ & -6.19500000 & -2.33100000 & -6.52400000 & $\mathrm{H}$ & -6.55500000 & -2.89100000 & -1.74800000 \\
\hline$S$ & -5.37800000 & -1.22800000 & -4.54500000 & C & -7.13000000 & -4.77100000 & -0.91100000 \\
\hline$S$ & -6.99000000 & -1.40100000 & -3.23100000 & C & -5.02900000 & -4.12300000 & -0.96600000 \\
\hline c & -8.26600000 & -1.18200000 & -7.61500000 & $\mathrm{H}$ & -4.15000000 & -3.50200000 & -1.15100000 \\
\hline 0 & -8.88600000 & -0.41600000 & -8.37800000 & $\mathrm{~N}$ & -5.00500000 & -5.31300000 & -0.40100000 \\
\hline $\mathrm{N}$ & -8.22900000 & -2.48900000 & -7.88200000 & C & -6.32900000 & -5.72100000 & -0.35800000 \\
\hline $\mathrm{H}$ & -7.74500000 & -3.15000000 & -7.27900000 & $\mathrm{H}$ & -6.59400000 & -6.68600000 & 0.05600000 \\
\hline C & -8.68100000 & -2.89700000 & -9.20500000 & C & -10.87100000 & -3.82700000 & -1.06100000 \\
\hline H & -9.76500000 & -2.78800000 & -9.29200000 & 0 & -11.74200000 & -4.07800000 & -0.22900000 \\
\hline $\mathrm{H}$ & -8.44100000 & -3.95300000 & -9.33900000 & $\mathrm{~N}$ & -8.88400000 & 8.39900000 & -0.21500000 \\
\hline C & -8.01500000 & -2.06400000 & -10.34000000 & $\mathrm{H}$ & -8.17200000 & 8.22200000 & 0.45800000 \\
\hline 0 & -8.67800000 & -2.04500000 & -11.42500000 & C & -9.27600000 & 7.31400000 & -1.10100000 \\
\hline 0 & -6.91300000 & -1.50800000 & -10.13200000 & $\mathrm{H}$ & -9.76500000 & 7.71400000 & -1.98600000 \\
\hline $\mathrm{Fe}$ & -7.64400000 & 0.87500000 & -2.57800000 & C & -8.06900000 & 6.44300000 & -1.54600000 \\
\hline 0 & -9.52600000 & 0.44400000 & -3.36800000 & $\mathrm{H}$ & -7.51300000 & 7.06300000 & -2.26000000 \\
\hline \multirow[t]{2}{*}{0} & -9.54100000 & 0.07000000 & -2.10300000 & $\mathrm{H}$ & -7.42600000 & 6.30400000 & -0.67300000 \\
\hline & & & & $\mathrm{N}$ & -9.19100000 & 4.83400000 & -3.20300000 \\
\hline \multicolumn{4}{|c|}{${ }^{7} \mathrm{R}^{\prime}$} & $\mathrm{H}$ & -9.72000000 & 5.53700000 & -3.74100000 \\
\hline \multicolumn{4}{|c|}{-4046.280883 (a.u.) } & C & -8.38200000 & 5.09000000 & -2.11000000 \\
\hline $\mathrm{N}$ & -4.78300000 & -0.53000000 & 3.76000000 & C & -9.23300000 & 3.50200000 & -3.40500000 \\
\hline $\mathrm{H}$ & -4.62000000 & -1.40300000 & 3.29600000 & $\mathrm{H}$ & -9.82000000 & 3.03300000 & -4.18000000 \\
\hline C & -6.16800000 & -0.15500000 & 3.91800000 & $\mathrm{~N}$ & -8.47300000 & 2.87500000 & -2.51000000 \\
\hline $\mathrm{H}$ & -6.36600000 & 0.04800000 & 4.96400000 & C & -7.95400000 & 3.85800000 & -1.69200000 \\
\hline C & -6.53600000 & 1.06400000 & 3.02800000 & $\mathrm{H}$ & -7.31300000 & 3.60800000 & -0.86300000 \\
\hline $\mathrm{H}$ & -7.55300000 & 1.39100000 & 3.24600000 & C & -10.31200000 & 6.45700000 & -0.40000000 \\
\hline $\mathrm{H}$ & -5.86800000 & 1.86000000 & 3.36900000 & 0 & -11.48100000 & 6.48000000 & -0.76100000 \\
\hline $\mathrm{N}$ & -5.26300000 & 0.51100000 & 0.85000000 & $\mathrm{~N}$ & -2.80600000 & 3.30200000 & -2.14900000 \\
\hline $\mathrm{H}$ & -4.33300000 & 0.27200000 & 1.21500000 & $\mathrm{H}$ & -3.29500000 & 3.26100000 & -1.26600000 \\
\hline C & -6.41600000 & 0.84800000 & 1.55400000 & C & -3.45200000 & 4.07500000 & -3.19500000 \\
\hline C & -5.56200000 & 0.47400000 & -0.45700000 & $\mathrm{H}$ & -3.01000000 & 3.84800000 & -4.16000000 \\
\hline $\mathrm{H}$ & -4.85000000 & 0.24600000 & -1.23400000 & C & -4.99000000 & 3.82700000 & -3.23400000 \\
\hline $\mathrm{N}$ & -6.84800000 & 0.76700000 & -0.64600000 & $\mathrm{H}$ & -5.34100000 & 3.80900000 & -2.20400000 \\
\hline C & -7.38900000 & 0.99500000 & 0.60200000 & $\mathrm{H}$ & -5.48700000 & 4.64800000 & -3.76000000 \\
\hline $\mathrm{H}$ & -8.43400000 & 1.22000000 & 0.74100000 & C & -5.39800000 & 2.55400000 & -3.98400000 \\
\hline C & -7.00000000 & -1.35400000 & 3.51700000 & $\mathrm{O}$ & -5.19600000 & 2.47200000 & -5.20500000 \\
\hline $\mathrm{O}$ & -6.43900000 & -2.41500000 & 3.24700000 & $\mathrm{O}$ & -6.00300000 & 1.65400000 & -3.28400000 \\
\hline $\mathrm{N}$ & -9.28200000 & -3.47300000 & 0.78200000 & C & -3.28700000 & 5.57400000 & -2.98500000 \\
\hline $\mathrm{H}$ & -9.20500000 & -4.31800000 & 1.30200000 & 0 & -3.62500000 & 6.36700000 & -3.86100000 \\
\hline C & -9.41900000 & -3.60800000 & -0.65600000 & $\mathrm{~N}$ & -7.16800000 & 1.22400000 & -9.59800000 \\
\hline $\mathrm{H}$ & -9.07500000 & -2.70500000 & -1.14200000 & $\mathrm{H}$ & -6.29900000 & 0.87300000 & -9.15000000 \\
\hline
\end{tabular}




\begin{tabular}{|c|c|c|c|c|c|c|c|}
\hline $\mathrm{H}$ & -7.12900000 & 0.88800000 & -10.56900000 & C & -6.53700000 & 1.07300000 & 2.98900000 \\
\hline $\mathrm{H}$ & -7.97500000 & 0.75500000 & -9.12600000 & $\mathrm{H}$ & -7.55600000 & 1.40800000 & 3.18600000 \\
\hline C & -7.01600000 & 2.70300000 & -9.47900000 & $\mathrm{H}$ & -5.87200000 & 1.88200000 & 3.31100000 \\
\hline $\mathrm{H}$ & -7.18700000 & 3.12600000 & -10.46800000 & $\mathrm{~N}$ & -5.24400000 & 0.45300000 & 0.83500000 \\
\hline C & -8.00000000 & 3.37700000 & -8.50900000 & $\mathrm{H}$ & -4.31500000 & 0.22900000 & 1.21200000 \\
\hline $\mathrm{H}$ & -9.02800000 & 3.14000000 & -8.80300000 & C & -6.39900000 & 0.82000000 & 1.52200000 \\
\hline $\mathrm{H}$ & -7.86200000 & 4.44500000 & -8.69900000 & C & -5.52400000 & 0.42800000 & -0.48000000 \\
\hline C & -7.81100000 & 3.11800000 & -6.99400000 & $\mathrm{H}$ & -4.80000000 & 0.20300000 & -1.24700000 \\
\hline $\mathrm{H}$ & -8.42400000 & 3.83500000 & -6.43800000 & $\mathrm{~N}$ & -6.80000000 & 0.74800000 & -0.68900000 \\
\hline $\mathrm{H}$ & -6.76700000 & 3.26800000 & -6.70500000 & C & -7.35100000 & 0.99000000 & 0.55300000 \\
\hline C & -8.30900000 & 1.73600000 & -6.59200000 & $\mathrm{H}$ & -8.39300000 & 1.24200000 & 0.67700000 \\
\hline $\mathrm{O}$ & -9.52000000 & 1.50600000 & -6.46400000 & C & -7.03500000 & -1.31300000 & 3.59800000 \\
\hline C & -5.52600000 & 2.98200000 & -9.09300000 & $\mathrm{O}$ & -6.49600000 & -2.38700000 & 3.33600000 \\
\hline $\mathrm{O}$ & -4.84800000 & 1.95800000 & -8.77800000 & $\mathrm{~N}$ & -9.74800000 & -3.49800000 & 1.06900000 \\
\hline 0 & -5.20100000 & 4.18200000 & -9.13600000 & $\mathrm{H}$ & -10.06900000 & -4.23900000 & 1.65300000 \\
\hline $\mathrm{N}$ & -7.33000000 & 0.80700000 & -6.49900000 & C & -9.78200000 & -3.72600000 & -0.36300000 \\
\hline $\mathrm{H}$ & -6.39100000 & 1.16700000 & -6.31600000 & $\mathrm{H}$ & -9.52900000 & -2.81400000 & -0.88500000 \\
\hline C & -7.52100000 & -0.63600000 & -6.38100000 & C & -8.78500000 & -4.82800000 & -0.80500000 \\
\hline $\mathrm{H}$ & -8.15400000 & -0.86600000 & -5.51300000 & $\mathrm{H}$ & -8.99000000 & -5.76700000 & -0.25000000 \\
\hline C & -6.15100000 & -1.29800000 & -6.22300000 & $\mathrm{H}$ & -8.91000000 & -5.03800000 & -1.88900000 \\
\hline $\mathrm{H}$ & -5.41600000 & -0.81000000 & -6.87200000 & $\mathrm{~N}$ & -6.85000000 & -3.19500000 & -0.44800000 \\
\hline $\mathrm{H}$ & -6.19100000 & -2.33600000 & -6.52700000 & $\mathrm{H}$ & -7.41700000 & -2.38600000 & -0.25300000 \\
\hline$S$ & -5.38400000 & -1.23300000 & -4.54000000 & C & -7.33400000 & -4.47500000 & -0.62000000 \\
\hline$S$ & -7.02100000 & -1.38900000 & -3.25200000 & C & -5.50100000 & -3.27900000 & -0.40600000 \\
\hline C & -8.25900000 & -1.18100000 & -7.62000000 & $\mathrm{H}$ & -4.84800000 & -2.41900000 & -0.25000000 \\
\hline 0 & -8.87900000 & -0.41400000 & -8.38200000 & $\mathrm{~N}$ & -5.08500000 & -4.52400000 & -0.54200000 \\
\hline $\mathrm{N}$ & -8.22700000 & -2.48900000 & -7.88600000 & C & -6.23800000 & -5.27900000 & -0.67300000 \\
\hline $\mathrm{H}$ & -7.73900000 & -3.14900000 & -7.28400000 & $\mathrm{H}$ & -6.17800000 & -6.35300000 & -0.80100000 \\
\hline C & -8.68000000 & -2.89600000 & -9.20800000 & C & -11.17300000 & -4.07600000 & -0.85000000 \\
\hline $\mathrm{H}$ & -9.76400000 & -2.78600000 & -9.29400000 & 0 & -12.08000000 & -4.37100000 & -0.07400000 \\
\hline $\mathrm{H}$ & -8.44200000 & -3.95300000 & -9.34000000 & $\mathrm{~N}$ & -8.86000000 & 8.56000000 & -0.11400000 \\
\hline C & -8.01300000 & -2.06600000 & -10.34300000 & $\mathrm{H}$ & -8.17900000 & 8.39000000 & 0.59200000 \\
\hline 0 & -8.67700000 & -2.04500000 & -11.42800000 & C & -9.18400000 & 7.48400000 & -1.03500000 \\
\hline $\mathrm{O}$ & -6.91000000 & -1.51100000 & -10.13600000 & $\mathrm{H}$ & -9.64700000 & 7.88800000 & -1.93300000 \\
\hline $\mathrm{Fe}$ & -7.63100000 & 0.87800000 & -2.56200000 & C & -7.93400000 & 6.66300000 & -1.45000000 \\
\hline $\mathrm{O}$ & -9.53400000 & 0.39800000 & -3.26200000 & $\mathrm{H}$ & -7.36300000 & 7.32000000 & -2.11800000 \\
\hline \multirow[t]{2}{*}{0} & -9.53800000 & 0.07100000 & -1.99300000 & $\mathrm{H}$ & -7.32800000 & 6.51100000 & -0.55200000 \\
\hline & & & & $\mathrm{N}$ & -8.95600000 & 5.09400000 & -3.20200000 \\
\hline \multicolumn{2}{|c|}{${ }^{7} R_{-} a^{\prime}$} & & & $\mathrm{H}$ & -9.50800000 & 5.78800000 & -3.73100000 \\
\hline \multicolumn{2}{|c|}{-4046.981718 (a.u.) } & & & C & -8.19200000 & 5.32700000 & -2.07200000 \\
\hline $\mathrm{N}$ & -4.79700000 & -0.50700000 & 3.78400000 & C & -8.96400000 & 3.77300000 & -3.45300000 \\
\hline $\mathrm{H}$ & -4.64100000 & -1.39000000 & 3.34000000 & $\mathrm{H}$ & -9.53800000 & 3.32100000 & -4.24700000 \\
\hline C & -6.17900000 & -0.11100000 & 3.93100000 & $\mathrm{~N}$ & -8.21700000 & 3.12800000 & -2.55900000 \\
\hline $\mathrm{H}$ & -6.36800000 & 0.14500000 & 4.96600000 & C & -7.75100000 & 4.08900000 & -1.68600000 \\
\hline
\end{tabular}




\begin{tabular}{|c|c|c|c|c|c|c|c|}
\hline $\mathrm{H}$ & -7.13100000 & 3.82200000 & -0.84700000 & 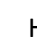 & -7.51400000 & -3.01200000 & -7.04700000 \\
\hline C & -10.22500000 & 6.58700000 & -0.39400000 & $c$ & -8.39600000 & -2.89300000 & -9.01000000 \\
\hline $\mathrm{O}$ & -11.37900000 & 6.59300000 & -0.80000000 & $r$ & -9.48700000 & -2.92400000 & -9.07200000 \\
\hline $\mathrm{N}$ & -2.78100000 & 3.26800000 & -2.14300000 & & -8.02500000 & -3.91400000 & -9.10400000 \\
\hline $\mathrm{H}$ & -3.26500000 & 3.22800000 & -1.25800000 & c & -7.85800000 & -2.03000000 & -10.18400000 \\
\hline C & -3.43700000 & 4.02900000 & -3.19100000 & c & -8.57000000 & -2.05400000 & -11.23600000 \\
\hline $\mathrm{H}$ & -2.97900000 & 3.82200000 & -4.15200000 & c & -6.78500000 & -1.40000000 & -10.03400000 \\
\hline C & -4.96300000 & 3.72400000 & -3.24600000 & & -7.61100000 & 1.08700000 & -2.57100000 \\
\hline $\mathrm{H}$ & -5.30700000 & 3.66700000 & -2.21700000 & c & -9.44900000 & 0.81800000 & -3.38000000 \\
\hline $\mathrm{H}$ & -5.48700000 & 4.54000000 & -3.75300000 & & -9.56100000 & 0.37000000 & -2.13200000 \\
\hline C & -5.31600000 & 2.45800000 & -4.02900000 & & & & \\
\hline 0 & -5.14800000 & 2.44700000 & -5.25900000 & \multicolumn{2}{|c|}{ 7TS1_a' } & & \\
\hline $\mathrm{O}$ & -5.83400000 & 1.47000000 & -3.36900000 & \multicolumn{2}{|c|}{-4046.943631 (a.u.) } & & \\
\hline C & -3.31400000 & 5.53100000 & -2.97000000 & $\mathrm{~N}$ & -4.79800000 & -0.52000000 & 3.69500000 \\
\hline $\mathrm{O}$ & -3.69900000 & 6.32100000 & -3.82900000 & $\mathrm{H}$ & -4.63400000 & -1.39000000 & 3.22800000 \\
\hline $\mathrm{N}$ & -7.19800000 & 1.22200000 & -9.53600000 & C & -6.18200000 & -0.14300000 & 3.85600000 \\
\hline $\mathrm{H}$ & -6.34600000 & 0.86400000 & -9.06300000 & $\mathrm{H}$ & -6.36500000 & 0.10800000 & 4.89500000 \\
\hline $\mathrm{H}$ & -7.15600000 & 0.80800000 & -10.47700000 & C & -6.56600000 & 1.03800000 & 2.92000000 \\
\hline $\mathrm{H}$ & -8.02200000 & 0.80300000 & -9.05700000 & $\mathrm{H}$ & -7.58200000 & 1.36600000 & 3.14200000 \\
\hline C & -7.01200000 & 2.70300000 & -9.52200000 & $\mathrm{H}$ & -5.89900000 & 1.85100000 & 3.22600000 \\
\hline $\mathrm{H}$ & -7.18100000 & 3.06000000 & -10.53800000 & $\mathrm{~N}$ & -5.32700000 & 0.44400000 & 0.72800000 \\
\hline C & -7.96900000 & 3.46200000 & -8.59300000 & $\mathrm{H}$ & -4.39100000 & 0.22700000 & 1.08600000 \\
\hline $\mathrm{H}$ & -9.00700000 & 3.25600000 & -8.87300000 & C & -6.46800000 & 0.78800000 & 1.44900000 \\
\hline $\mathrm{H}$ & -7.78600000 & 4.51500000 & -8.82300000 & C & -5.64500000 & 0.41900000 & -0.57900000 \\
\hline C & -7.78400000 & 3.24600000 & -7.07500000 & $\mathrm{H}$ & -4.94100000 & 0.20900000 & -1.36900000 \\
\hline $\mathrm{H}$ & -8.33000000 & 4.02800000 & -6.53600000 & $\mathrm{~N}$ & -6.93200000 & 0.71700000 & -0.75300000 \\
\hline $\mathrm{H}$ & -6.73000000 & 3.31600000 & -6.79300000 & C & -7.45100000 & 0.94200000 & 0.50700000 \\
\hline C & -8.41000000 & 1.93600000 & -6.63100000 & $\mathrm{H}$ & -8.49300000 & 1.17000000 & 0.66500000 \\
\hline $\mathrm{O}$ & -9.64200000 & 1.80300000 & -6.59700000 & C & -7.01800000 & -1.35900000 & 3.52700000 \\
\hline C & -5.51700000 & 2.97900000 & -9.15400000 & 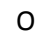 & -6.46200000 & -2.42200000 & 3.25100000 \\
\hline 0 & -4.84600000 & 1.96200000 & -8.80500000 & $\mathrm{~N}$ & -9.60500000 & -3.61100000 & 1.01700000 \\
\hline $\mathrm{O}$ & -5.18100000 & 4.17400000 & -9.23800000 & $\mathrm{H}$ & -9.78100000 & -4.40400000 & 1.59300000 \\
\hline $\mathrm{N}$ & -7.52100000 & 0.95600000 & -6.36400000 & C & -9.66700000 & -3.81000000 & -0.41900000 \\
\hline $\mathrm{H}$ & -6.54600000 & 1.23800000 & -6.25400000 & $\mathrm{H}$ & -9.35700000 & -2.90500000 & -0.91700000 \\
\hline C & -7.87200000 & -0.43800000 & -6.10000000 & $c$ & -8.73800000 & -4.95200000 & -0.88900000 \\
\hline $\mathrm{H}$ & -8.80400000 & -0.45500000 & -5.51900000 & $\mathrm{H}$ & -9.01400000 & -5.89900000 & -0.38000000 \\
\hline C & -6.74000000 & -1.08500000 & -5.29100000 & п & -8.85100000 & -5.11000000 & -1.98300000 \\
\hline $\mathrm{H}$ & -5.84400000 & -0.46100000 & -5.28400000 & $\mathrm{~N}$ & -6.68200000 & -3.46100000 & -0.66900000 \\
\hline $\mathrm{H}$ & -6.45600000 & -2.06300000 & -5.67100000 & $\mathrm{H}$ & -7.17300000 & -2.58700000 & -0.69500000 \\
\hline$S$ & -7.15300000 & -1.33000000 & -3.51300000 & C & -7.27600000 & -4.70600000 & -0.65500000 \\
\hline$S$ & -8.88800000 & -2.37500000 & -3.53300000 & $c$ & -5.34800000 & & -0.55100000 \\
\hline C & -8.26600000 & -1.11500000 & -7.43300000 & $\mathrm{H}$ & -4.61900000 & -2.84200000 & -0.52000000 \\
\hline $\mathrm{O}$ & -8.91100000 & -0.43500000 & -8.25600000 & $\mathrm{~N}$ & -5.04600000 & -4.93700000 & -0.46700000 \\
\hline & -7.98300000 & -2.38700000 & -7.70200000 & C & -6.26000000 & -5.60000000 & -0.52400000 \\
\hline
\end{tabular}




\begin{tabular}{|c|c|c|c|c|c|c|c|}
\hline $\mathrm{H}$ & -6.29900000 & -6.68200000 & -0.48300000 & C & -8.38900000 & 1.87100000 & -6.68600000 \\
\hline C & -11.07500000 & -4.06900000 & -0.91800000 & $\mathrm{O}$ & -9.61600000 & 1.73100000 & -6.73400000 \\
\hline 0 & -11.99700000 & -4.33200000 & -0.14700000 & C & -5.50400000 & 2.92800000 & -9.14800000 \\
\hline N & -8.86000000 & 8.59800000 & -0.07200000 & 0 & -4.85900000 & 1.90100000 & -8.77900000 \\
\hline $\mathrm{H}$ & -8.18700000 & 8.43000000 & 0.64200000 & $\mathrm{O}$ & -5.14200000 & 4.11400000 & -9.25000000 \\
\hline C & -9.18800000 & 7.51600000 & -0.98300000 & $\mathrm{~N}$ & -7.48000000 & 0.90000000 & -6.43300000 \\
\hline $\mathrm{H}$ & -9.65900000 & 7.91400000 & -1.87900000 & $\mathrm{H}$ & -6.54000000 & 1.23100000 & -6.21300000 \\
\hline C & -7.94400000 & 6.69000000 & -1.41100000 & C & -7.77400000 & -0.50400000 & -6.13600000 \\
\hline $\mathrm{H}$ & -7.39200000 & 7.32800000 & -2.11200000 & $\mathrm{H}$ & -8.60800000 & -0.53700000 & -5.42000000 \\
\hline $\mathrm{H}$ & -7.31400000 & 6.55900000 & -0.52600000 & C & -6.51300000 & -1.15500000 & -5.53400000 \\
\hline $\mathrm{N}$ & -9.07300000 & 5.08800000 & -3.06200000 & $\mathrm{H}$ & -5.66100000 & -0.47400000 & -5.60400000 \\
\hline $\mathrm{H}$ & -9.59700000 & 5.78000000 & -3.62000000 & $\mathrm{H}$ & -6.23800000 & -2.06000000 & -6.06900000 \\
\hline C & -8.23900000 & 5.33900000 & -1.98400000 & $S$ & -6.54300000 & -1.57300000 & -3.72800000 \\
\hline C & -9.16800000 & 3.75900000 & -3.23500000 & $\mathrm{~S}$ & -8.45100000 & -2.19500000 & -3.31900000 \\
\hline $\mathrm{H}$ & -9.78200000 & 3.28900000 & -3.98800000 & C & -8.29800000 & -1.18200000 & -7.42300000 \\
\hline $\mathrm{N}$ & -8.41400000 & 3.12500000 & -2.33700000 & 0 & -8.95400000 & -0.50900000 & -8.23500000 \\
\hline C & -7.84500000 & 4.10200000 & -1.54900000 & $\mathrm{~N}$ & -8.10300000 & -2.48800000 & -7.66600000 \\
\hline $\mathrm{H}$ & -7.19300000 & 3.83900000 & -0.73400000 & $\mathrm{H}$ & -7.51100000 & -3.07100000 & -7.07800000 \\
\hline C & -10.22700000 & 6.62300000 & -0.32900000 & C & -8.47800000 & -2.95500000 & -9.00200000 \\
\hline O & -11.38700000 & 6.64500000 & -0.71600000 & $\mathrm{H}$ & -9.56500000 & -2.96000000 & -9.10600000 \\
\hline $\mathrm{N}$ & -2.83300000 & 3.26600000 & -2.15200000 & $\mathrm{H}$ & -8.12500000 & -3.98100000 & -9.10900000 \\
\hline $\mathrm{H}$ & -3.31500000 & 3.22300000 & -1.26600000 & C & -7.88700000 & -2.07500000 & -10.14400000 \\
\hline C & -3.48900000 & 4.03100000 & -3.19900000 & $\mathrm{O}$ & -8.57200000 & -2.06500000 & -11.21400000 \\
\hline $\mathrm{H}$ & -3.03500000 & 3.81800000 & -4.16100000 & 0 & -6.80800000 & -1.47100000 & -9.94700000 \\
\hline C & -5.02100000 & 3.74900000 & -3.25500000 & $\mathrm{Fe}$ & -7.80000000 & 1.13000000 & -2.63400000 \\
\hline $\mathrm{H}$ & -5.37800000 & 3.70500000 & -2.22800000 & $\mathrm{O}$ & -9.22500000 & 0.98500000 & -3.80800000 \\
\hline $\mathrm{H}$ & -5.53300000 & 4.57000000 & -3.76900000 & $\mathrm{O}$ & -9.17700000 & -0.35700000 & -2.82100000 \\
\hline C & -5.39900000 & 2.48600000 & -4.03200000 & & & & \\
\hline 0 & -5.13500000 & 2.40400000 & -5.24100000 & \multicolumn{2}{|c|}{ 'IM1_a' } & & \\
\hline 0 & -6.04600000 & 1.57300000 & -3.38200000 & \multicolumn{2}{|c|}{-4047.020826 (a.u.) } & & \\
\hline C & -3.34800000 & 5.53200000 & -2.98100000 & $\mathrm{~N}$ & -4.87600000 & -0.53400000 & 3.72200000 \\
\hline 0 & -3.71800000 & 6.32300000 & -3.84500000 & $\mathrm{H}$ & -4.72000000 & -1.40700000 & 3.25800000 \\
\hline $\mathrm{N}$ & -7.22400000 & 1.20200000 & -9.52000000 & C & -6.25600000 & -0.13200000 & 3.87000000 \\
\hline $\mathrm{H}$ & -6.39100000 & 0.83000000 & -9.02400000 & $\mathrm{H}$ & -6.44300000 & 0.13000000 & 4.90500000 \\
\hline $\mathrm{H}$ & -7.16900000 & 0.79000000 & -10.46000000 & C & -6.60500000 & 1.05300000 & 2.92500000 \\
\hline $\mathrm{H}$ & -8.06400000 & 0.80600000 & -9.05700000 & $\mathrm{H}$ & -7.61900000 & 1.40000000 & 3.12800000 \\
\hline C & -7.00700000 & 2.68200000 & -9.51100000 & $\mathrm{H}$ & -5.92900000 & 1.85700000 & 3.24000000 \\
\hline H & -7.17500000 & 3.03900000 & -10.52700000 & $\mathrm{~N}$ & -5.34300000 & 0.40500000 & 0.75800000 \\
\hline C & -7.94000000 & 3.46300000 & -8.57500000 & $\mathrm{H}$ & -4.41000000 & 0.18500000 & 1.12500000 \\
\hline $\mathrm{H}$ & -8.98300000 & 3.29400000 & -8.86000000 & C & -6.48400000 & 0.79100000 & 1.45700000 \\
\hline $\mathrm{H}$ & -7.71900000 & 4.51100000 & -8.79200000 & C & -5.64400000 & 0.37100000 & -0.55400000 \\
\hline C & -7.76700000 & 3.20600000 & -7.06400000 & $\mathrm{H}$ & -4.93200000 & 0.14000000 & -1.33100000 \\
\hline H & -8.32900000 & 3.96100000 & -6.50400000 & $\mathrm{~N}$ & -6.91900000 & 0.70200000 & -0.75100000 \\
\hline H & -6.71500000 & 3.28000000 & -6.77300000 & C & -7.44700000 & 0.96300000 & 0.49800000 \\
\hline
\end{tabular}




\begin{tabular}{|c|c|c|c|c|c|c|c|}
\hline $\mathrm{H}$ & -8.48300000 & 1.22800000 & 0.63700000 & C & -5.28500000 & 2.43100000 & -4.06900000 \\
\hline c & -7.12900000 & -1.32400000 & 3.54000000 & $\mathrm{O}$ & -5.04300000 & 2.40600000 & -5.29000000 \\
\hline 0 & -6.60500000 & -2.39800000 & 3.24800000 & $\mathrm{O}$ & -5.85000000 & 1.46300000 & -3.43100000 \\
\hline $\mathrm{N}$ & -9.98600000 & -3.43600000 & 1.06400000 & C & -3.33300000 & 5.53500000 & -2.97500000 \\
\hline $\mathrm{H}$ & -10.32900000 & -4.17100000 & 1.64200000 & $\mathrm{O}$ & -3.72700000 & 6.33100000 & -3.82500000 \\
\hline C & -10.02600000 & -3.65500000 & -0.37000000 & $\mathrm{~N}$ & -7.20200000 & 1.24300000 & -9.49700000 \\
\hline $\mathrm{H}$ & -9.79100000 & -2.73500000 & -0.88600000 & $\mathrm{H}$ & -6.36000000 & 0.87400000 & -9.01300000 \\
\hline C & -9.02000000 & -4.74600000 & -0.81900000 & $\mathrm{H}$ & -7.16300000 & 0.81600000 & -10.43100000 \\
\hline $\mathrm{H}$ & -9.19600000 & -5.67700000 & -0.24100000 & $\mathrm{H}$ & -8.03600000 & 0.85900000 & -9.01600000 \\
\hline $\mathrm{H}$ & -9.17000000 & -4.99000000 & -1.89300000 & C & -6.98100000 & 2.72300000 & -9.51200000 \\
\hline $\mathrm{N}$ & -7.07300000 & -3.11600000 & -0.46800000 & $\mathrm{H}$ & -7.17600000 & 3.06700000 & -10.52700000 \\
\hline $\mathrm{H}$ & -7.62300000 & -2.31200000 & -0.21000000 & C & -7.88400000 & 3.51400000 & -8.55700000 \\
\hline C & -7.56700000 & -4.38700000 & -0.67700000 & $\mathrm{H}$ & -8.93600000 & 3.34700000 & $-8.8080000 c$ \\
\hline C & -5.72200000 & -3.20800000 & -0.47600000 & $\mathrm{H}$ & -7.66600000 & 4.56000000 & -8.78600000 \\
\hline $\mathrm{H}$ & -5.06100000 & -2.35500000 & -0.30600000 & C & -7.66400000 & 3.26400000 & $-7.0520000 c$ \\
\hline$N$ & -5.31600000 & -4.44700000 & -0.67900000 & $\mathrm{H}$ & -8.19700000 & 4.02800000 & -6.47600000 \\
\hline C & -6.47800000 & -5.19100000 & -0.79900000 & $\mathrm{H}$ & -6.60400000 & 3.32100000 & $-6.7920000 c$ \\
\hline $\mathrm{H}$ & -6.43400000 & -6.26100000 & -0.96800000 & C & -8.29500000 & 1.94200000 & -6.64800000 \\
\hline C & -11.40300000 & -4.04900000 & -0.86500000 & $\mathrm{O}$ & -9.52700000 & 1.82200000 & -6.66700000 \\
\hline 0 & -12.25300000 & -4.53100000 & -0.11800000 & C & -5.47000000 & 2.96800000 & -9.19200000 \\
\hline $\mathrm{N}$ & -8.83800000 & 8.65900000 & -0.07500000 & 0 & -4.81400000 & 1.94300000 & -8.83900000 \\
\hline $\mathrm{H}$ & -8.16000000 & 8.48900000 & 0.63300000 & 0 & -5.11100000 & 4.15300000 & -9.30900000 \\
\hline C & -9.14900000 & 7.58800000 & -1.00500000 & $\mathrm{~N}$ & -7.40600000 & 0.95300000 & -6.40700000 \\
\hline $\mathrm{H}$ & -9.61500000 & 7.99300000 & -1.90000000 & $\mathrm{H}$ & -6.44500000 & 1.25200000 & -6.22600000 \\
\hline C & -7.89400000 & 6.78000000 & -1.43100000 & C & -7.76900000 & -0.42600000 & -6.07100000 \\
\hline $\mathrm{H}$ & -7.33800000 & 7.43700000 & -2.11200000 & $\mathrm{H}$ & -8.62000000 & -0.36000000 & -5.3730000 \\
\hline $\mathrm{H}$ & -7.27600000 & 6.63900000 & -0.54000000 & C & -6.56200000 & -1.10800000 & -5.39000000 \\
\hline $\mathrm{N}$ & -8.97700000 & 5.17300000 & -3.11800000 & $\mathrm{H}$ & -5.77800000 & -0.37300000 & -5.1940000 \\
\hline $\mathrm{H}$ & -9.53400000 & 5.85300000 & -3.65900000 & $\mathrm{H}$ & -6.13300000 & -1.88900000 & -6.0140000 \\
\hline C & -8.16100000 & 5.43500000 & -2.03000000 & & -6.84800000 & -1.80000000 & -3.6990000 \\
\hline C & -9.01800000 & 3.84300000 & -3.31800000 & $\mathrm{~s}$ & -8.69400000 & -2.82000000 & -3.9740000 \\
\hline $\mathrm{H}$ & -9.61800000 & 3.35900000 & -4.07300000 & & -8.26500000 & -1.11900000 & -7.36000000 \\
\hline $\mathrm{N}$ & -8.24300000 & 3.22400000 & -2.43100000 & 0 & -8.92800000 & -0.46300000 & -8.18100000 \\
\hline C & -7.72000000 & 4.20600000 & -1.62000000 & $\mathrm{~N}$ & -8.02700000 & -2.41700000 & -7.61800000 \\
\hline $\mathrm{H}$ & -7.05800000 & 3.95900000 & -0.80900000 & $\mathrm{H}$ & -7.46000000 & -3.02300000 & -7.0210000 \\
\hline C & -10.18600000 & 6.67800000 & -0.37100000 & C & -8.40800000 & -2.88800000 & -8.9490000 \\
\hline 0 & -11.33200000 & 6.66100000 & -0.79900000 & $\mathrm{H}$ & -9.49700000 & -2.89200000 & -9.04700000 \\
\hline$N$ & -2.78700000 & 3.27100000 & -2.16100000 & $\mathrm{H}$ & -8.05100000 & -3.91300000 & -9.05400000 \\
\hline $\mathrm{H}$ & -3.28300000 & 3.21200000 & -1.28300000 & C & -7.85400000 & -2.02300000 & -10.1160000 \\
\hline C & -3.44100000 & 4.03300000 & -3.20900000 & $\mathrm{O}$ & -8.56500000 & -2.05100000 & -11.17100000 \\
\hline $\mathrm{H}$ & -2.97000000 & & -4.16700000 & $\mathrm{O}$ & -6.78200000 & -1.39900000 & $-9.9570000 c$ \\
\hline C & -4.96200000 & 3.70900000 & -3.28700000 & $\mathrm{Fe}$ & -7.69000000 & 1.20900000 & -2.64200000 \\
\hline $\mathrm{H}$ & -5.32600000 & 3.65300000 & -2.26400000 & $\mathrm{O}$ & -9.01700000 & 0.66500000 & -3.55900000 \\
\hline "r & -5.48600000 & 4.51600000 & -3.80700000 & 0 & -9.79900000 & -2.07200000 & -3.24900000 \\
\hline
\end{tabular}


7TS2_a'

-4047.007701 (a.u.)

N $\quad-4.84300000$

H $\quad-4.67300000$

C -6.23100000

H $\quad-6.43900000$

C -6.59300000

H $\quad-7.61500000$

H $\quad-5.93500000$

N $\quad-5.29000000$

H -4.37400000

C -6.44900000

C $\quad-5.55500000$

H -4.83100000

N $\quad-6.82600000$

C $\quad-7.39100000$

H $\quad-8.43100000$

C $\quad-7.06200000$

O -6.50100000

N $\quad-9.52500000$

H $\quad-9.59700000$

C $\quad-9.62000000$

H $\quad-9.27800000$

C $\quad-8.74200000$

H $\quad-9.04500000$

H $\quad-8.89700000$

N -6.50500000

H $\quad-6.82600000$

C $\quad-7.26600000$

C $\quad-5.21900000$

H $\quad-4.38600000$

N $\quad-5.10300000$

C $\quad-6.39200000$

H -6.58200000

C -11.04800000

O -11.95800000

N $\quad-8.85800000$

H $\quad-8.18600000$

C $\quad-9.16500000$

H $\quad-9.62500000$

C $\quad-7.90300000$

H $\quad-7.33600000$

H $\quad-7.30000000$
$-0.53500000$

3.68400000

$-1.40300000$

3.21400000

$-0.15600000$

0.06200000

1.05200000

1.37400000

1.85700000

0.49300000

0.22500000

0.84500000

0.51100000

0.28600000

0.85500000

1.05700000

1.31400000

$-1.36000000$

$-2.42200000$

$-3.60500000$

$-4.41400000$

$-3.81300000$

$-2.92600000$

$-4.99000000$

$-5.91300000$

$-5.17000000$

$-3.81400000$

$-3.04900000$

$-4.80900000$

$-4.03700000$

$-3.40100000$

$-5.10300000$

$-5.59000000$

$-6.48300000$

$-4.07100000$

$-4.28700000$

8.63700000

8.46700000

7.56500000

7.97000000

6.76000000

7.42300000

6.61200000
3.82100000

4.86200000

2.91100000

3.11300000

3.26000000

0.74900000

1.13000000

1.43700000

$-0.56700000$

$-0.77900000$

0.46500000

0.58900000

3.43200000

3.16800000

0.82800000

1.40500000

$-0.60500000$

$-1.11000000$

$-1.07600000$

$-0.53800000$

$-2.16000000$

$-1.44900000$

$-2.01500000$

$-0.87100000$

$-1.08700000$

$-1.39800000$

$-0.32200000$

$-0.18200000$

0.40100000

$-1.07000000$

$-0.27000000$

$-0.07300000$

0.64100000

$-1.00400000$

$-1.90200000$

$-1.41500000$

$-2.08000000$

$-0.51500000$
$-1.33400000$

H $\quad-7.21900000$
N $\quad-8.94000000$

H $\quad-9.50400000$

C $\quad-8.15000000$

5.17700000

$-3.14700000$

5.86100000

$-3.67700000$

C $\quad-8.94600000$

H $\quad-9.52700000$

N $\quad-8.17300000$

C $\quad-7.68800000$

H $\quad-7.03800000$

C -10.20300000

O -11.35100000

N $\quad-2.74500000$

H $\quad-3.22200000$

C -3.38300000

H $\quad-2.91100000$

C -4.90700000

H $\quad-5.26000000$

H $\quad-5.43800000$

C $\quad-5.23800000$

O -4.95100000

O -5.85800000

C $\quad-3.26400000$

O -3.64800000

N $\quad-7.14200000$

H $\quad-6.27100000$

H $\quad-7.12000000$

H $\quad-7.94900000$

C $\quad-6.98600000$

C $\quad-7.89900000$

H $\quad-8.94900000$

H $\quad-7.72400000$

C $\quad-7.63500000$

H $\quad-8.12500000$

H $\quad-6.56700000$

C $\quad-8.28900000$

O -9.52500000

C $\quad-5.48000000$

O -4.76500000

O -5.17500000

N $\quad-7.43800000$

H $\quad-6.44500000$

C $\quad-7.86000000$

H $\quad-8.81500000$

C -6.80300000
$5.42200000 \quad-2.03700000$

$3.85400000-3.39000000$

$3.39100000-4.17300000$

$3.22300000-2.51000000$

$4.19000000-1.65700000$

$3.93200000-0.84000000$

$6.65500000-0.37500000$

$6.64600000-0.79800000$

$3.38300000-2.12900000$

$3.35900000-1.24000000$

$4.16500000-3.17200000$

$3.97500000-4.13000000$

$3.86800000-3.25100000$

$3.75100000-2.22900000$

$4.70600000-3.71100000$

2.64900000

$-4.11100000$

$2.66500000-5.31700000$

$1.66600000-3.54100000$

$5.66100000-2.93200000$

$6.46200000-3.78200000$

$1.26900000-9.51100000$

$0.93000000-9.06200000$

$0.84300000-10.44700000$

$0.84100000-9.00700000$

$2.75200000-9.51400000$

$3.10100000-10.51900000$

$3.49000000 \quad-8.52700000$

$4.54600000-8.74700000$

$3.23600000 \quad-7.02700000$

$4.02400000-6.44400000$

$3.26900000-6.79900000$

$1.94400000-6.56800000$

$1.85400000-6.54100000$

$3.05900000-9.23000000$

$2.05900000-8.92700000$

$4.26100000 \quad-9.32800000$

$0.93600000-6.27000000$

$1.15600000-6.21100000$

$-0.44300000-6.03000000$

$-0.39300000-5.50300000$

$-1.18900000 \quad-5.19600000$
$3.28700000-8.75800000$ 


\begin{tabular}{|c|c|c|c|c|c|c|c|}
\hline $\mathrm{H}$ & -5.81500000 & -0.75300000 & -5.36300000 & $\mathrm{~N}$ & -6.59300000 & -3.69900000 & -1.46200000 \\
\hline $\mathrm{H}$ & -6.74900000 & -2.22900000 & -5.49400000 & $\mathrm{H}$ & -6.94600000 & -2.93200000 & -2.00700000 \\
\hline S & -7.02000000 & -1.21600000 & -3.36000000 & C & -7.31200000 & -4.72100000 & -0.88000000 \\
\hline S & -9.48800000 & -1.39000000 & -3.25500000 & C & -5.29200000 & -3.89500000 & -1.14200000 \\
\hline C & -8.23200000 & -1.07200000 & -7.39800000 & $\mathrm{H}$ & -4.48800000 & -3.23300000 & -1.47000000 \\
\hline 0 & -8.84800000 & -0.35200000 & -8.21100000 & $\mathrm{~N}$ & -5.12700000 & -4.97000000 & -0.39900000 \\
\hline$N$ & -7.95800000 & -2.33100000 & -7.72500000 & C & -6.39900000 & -5.49200000 & -0.22900000 \\
\hline $\mathrm{H}$ & -7.56300000 & -3.01300000 & -7.07300000 & $\mathrm{H}$ & -6.55000000 & -6.39900000 & 0.34400000 \\
\hline C & -8.41200000 & -2.79400000 & -9.03200000 & C & -11.08100000 & -3.99300000 & -1.06500000 \\
\hline $\mathrm{H}$ & -9.50400000 & -2.76800000 & -9.07300000 & 0 & -11.97900000 & -4.28800000 & -0.27800000 \\
\hline $\mathrm{H}$ & -8.09200000 & -3.83100000 & -9.14800000 & $\mathrm{~N}$ & -8.85500000 & 8.49200000 & -0.14800000 \\
\hline C & -7.87700000 & -1.95600000 & 10.22000000 & $\mathrm{H}$ & -8.16600000 & 8.32500000 & 0.55100000 \\
\hline $\mathrm{O}$ & -8.60400000 & -2.00900000 & -11.26300000 & C & -9.17900000 & 7.41000000 & -1.06400000 \\
\hline 0 & -6.80100000 & -1.32900000 & -10.09600000 & $\mathrm{H}$ & -9.62300000 & 7.81300000 & -1.97100000 \\
\hline$F$ & -7.56700000 & 1.19300000 & -2.68500000 & C & -7.92800000 & 6.57100000 & $-1.4480000 c$ \\
\hline $\mathrm{O}$ & -9.19300000 & 0.61000000 & -3.16100000 & $\mathrm{H}$ & -7.33500000 & 7.22800000 & -2.09700000 \\
\hline \multirow[t]{2}{*}{ O } & -9.48000000 & -2.57700000 & -4.21700000 & $\mathrm{H}$ & -7.34500000 & 6.41500000 & $-0.5350000 c$ \\
\hline & & & & $\mathrm{N}$ & -8.93000000 & 5.01100000 & -3.21700000 \\
\hline \multicolumn{2}{|c|}{ 'IM2_a' } & & & $\mathrm{H}$ & -9.49800000 & 5.70500000 & -3.72700000 \\
\hline \multicolumn{2}{|c|}{-4047.067259 (a.u.) } & & & C & -8.16500000 & 5.23800000 & -2.08800000 \\
\hline$N$ & -4.83000000 & -0.51600000 & 3.70900000 & $\mathrm{C}$ & -8.87900000 & 3.69600000 & -3.51100000 \\
\hline $\mathrm{H}$ & -4.65700000 & -1.39100000 & 3.25500000 & $\mathrm{H}$ & -9.43400000 & 3.25400000 & -4.32300000 \\
\hline C & -6.21800000 & -0.12400000 & 3.81100000 & $\mathrm{~N}$ & -8.09700000 & 3.05400000 & -2.64900000 \\
\hline $\mathrm{H}$ & -6.44500000 & 0.12400000 & 4.84300000 & C & -7.66400000 & 4.00700000 & $-1.7500000 c$ \\
\hline C & -6.55200000 & 1.06400000 & 2.86300000 & $\mathrm{H}$ & -7.02100000 & 3.74200000 & -0.92700000 \\
\hline $\mathrm{H}$ & -7.57800000 & 1.39400000 & 3.02800000 & C & -10.23900000 & 6.53000000 & -0.42900000 \\
\hline $\mathrm{H}$ & -5.90000000 & 1.87400000 & 3.20800000 & 0 & -11.39600000 & 6.57500000 & -0.82500000 \\
\hline $\mathrm{N}$ & -5.17700000 & 0.44800000 & 0.76300000 & $\mathrm{~N}$ & -2.74600000 & 3.49000000 & -2.20000000 \\
\hline $\mathrm{H}$ & -4.26800000 & 0.20700000 & 1.17800000 & $\mathrm{H}$ & -3.26800000 & 3.46600000 & -1.33500000 \\
\hline C & -6.36200000 & 0.81400000 & & & & & -3.2660000 \\
\hline C & -5.39700000 & 0.40300000 & -0.55700000 & $\mathrm{H}$ & -2.80100000 & 4.09300000 & -4.20300000 \\
\hline $\mathrm{H}$ & -4.64900000 & 0.15400000 & -1.29200000 & C & -4.83300000 & 4.09100000 & -3.41500000 \\
\hline $\mathrm{N}$ & -6.66600000 & 0.72300000 & -0.82300000 & $\mathrm{H}$ & -5.26200000 & 4.13300000 & -2.41700000 \\
\hline C & -7.27600000 & 0.97600000 & 0.39300000 & $\mathrm{H}$ & -5.26100000 & 4.88800000 & -4.02600000 \\
\hline $\mathrm{H}$ & -8.32100000 & 1.23200000 & 0.47000000 & $\mathrm{C}$ & -5.18700000 & 2.78100000 & $-4.1100000 c$ \\
\hline C & -7.06100000 & -1.32400000 & 3.43500000 & 0 & -5.06600000 & 2.68400000 & -5.34000000 \\
\hline $\mathrm{O}$ & -6.51000000 & -2.39200000 & 3.17200000 & 0 & -5.63900000 & 1.83600000 & -3.35600000 \\
\hline N & -9.56600000 & -3.52800000 & 0.83900000 & C & -3.14600000 & 5.79200000 & -3.01100000 \\
\hline $\mathrm{H}$ & -9.64600000 & -4.33600000 & 1.41600000 & 0 & -3.48500000 & 6.60900000 & -3.86600000 \\
\hline C & -9.65600000 & -3.73800000 & -0.59300000 & $\mathrm{~N}$ & -7.10200000 & 1.28800000 & -9.49500000 \\
\hline $\mathrm{H}$ & -9.29900000 & -2.86200000 & -1.10700000 & $\mathrm{H}$ & -6.21800000 & 0.97300000 & -9.04900000 \\
\hline C & -8.78800000 & -4.92600000 & -1.04700000 & $\mathrm{H}$ & -7.07900000 & 0.87500000 & -10.43600000 \\
\hline $\mathrm{H}$ & -9.07300000 & -5.83500000 & -0.47500000 & $\mathrm{H}$ & -7.89700000 & 0.84000000 & $-8.9870000 c$ \\
\hline & -8.96700000 & -5.14000000 & -2.12100000 & C & -6.98300000 & 2.77100000 & -9.472000 \\
\hline
\end{tabular}




\begin{tabular}{llll}
$\mathrm{H}$ & -7.19400000 & 3.13200000 & -10.47800000 \\
$\mathrm{C}$ & -7.95500000 & 3.46500000 & -8.50800000 \\
$\mathrm{H}$ & -8.98700000 & 3.21700000 & -8.77500000 \\
$\mathrm{H}$ & -7.82400000 & 4.52800000 & -8.72700000 \\
$\mathrm{C}$ & -7.73300000 & 3.24000000 & -6.99500000 \\
$\mathrm{H}$ & -8.25900000 & 4.03100000 & -6.44900000 \\
$\mathrm{H}$ & -6.67300000 & 3.31200000 & -6.74000000 \\
$\mathrm{C}$ & -8.35800000 & 1.94000000 & -6.50100000 \\
$\mathrm{O}$ & -9.59000000 & 1.81900000 & -6.46000000 \\
$\mathrm{C}$ & -5.48900000 & 3.10400000 & -9.14600000 \\
$\mathrm{O}$ & -4.76700000 & 2.11000000 & -8.83400000 \\
$\mathrm{O}$ & -5.20000000 & 4.31100000 & -9.23000000 \\
$\mathrm{~N}$ & -7.46900000 & 0.97500000 & -6.15600000 \\
$\mathrm{H}$ & -6.48800000 & 1.25600000 & -6.12600000 \\
$\mathrm{C}$ & -7.77100000 & -0.45100000 & -5.99000000 \\
$\mathrm{H}$ & -8.66700000 & -0.56900000 & -5.37300000 \\
$\mathrm{C}$ & -6.56800000 & -1.12500000 & -5.30500000 \\
$\mathrm{H}$ & -7.29000000 & 0.99300000 & -2.75400000 \\
$\mathrm{H}$ & -5.64400000 & -0.64800000 & -5.64200000 \\
$\mathrm{H}$ & -6.50400000 & -2.17000000 & -5.59300000 \\
$\mathrm{~S}$ & -6.56700000 & -1.13400000 & -3.46200000 \\
$\mathrm{H}$ & -9.94800000 & -0.79100000 & -2.66000000 \\
$\mathrm{C}$ & -8.17300000 & -1.02300000 & -7.37400000 \\
$\mathrm{O}$ & -8.79200000 & -0.28300000 & -8.16700000 \\
$\mathrm{H}$ & -7.92200000 & -2.28100000 & -7.72500000 \\
$\mathrm{H}$ & -7.52900000 & -2.97100000 & -7.08000000 \\
$\mathrm{H}$ & -8.40900000 & -2.72900000 & -9.02400000 \\
\hline & -9.50100000 & -2.67000000 & -9.05100000 \\
$\mathrm{H}$ & -7.86600000 & -1.91200000 & -10.22100000 \\
\hline
\end{tabular}

